\title{
2018 ASSBI 41st Annual Conference Abstracts
}

Kevin Walsh Award for Most Outstanding Masters Student 2018

\section{A Scoping Review of the Working Alliance in Acquired Brain Injury Rehabilitation}

Kellie Stagg, ${ }^{1,2}$ Jacinta Douglas, ${ }^{2,3}$ and Teresa lacono ${ }^{1,2}$

${ }^{1}$ La Trobe Rural Health School, La Trobe University, Bendigo, Australia

${ }^{2}$ Living with Disability Research Centre, School of Allied Health, La Trobe University, Melbourne, Australia

${ }^{3}$ Summer Foundation, Melbourne, Australia

$\boldsymbol{B}$ ackground and aims: The aim was to review the empirical literature to determine the nature and breadth of research into the working alliance (WA) in acquired brain injury (ABI) rehabilitation.

Method: A scoping review was conducted, beginning with a systematic search of relevant databases using key search terms. Studies with a focus on the role of the WA in shaping rehabilitation outcomes, and factors influencing perceptions of the WA were included and key information extracted.

Results: A total of 10 quantitative studies met inclusion criteria. In most studies, ratings of the WA were compared with other process variables or outcome measures. The WA was linked to positive activity and participation outcomes, including return to work, school and driving. Client-related factors, such as age, level of education and approach to rehabilitation tasks were associated with client and therapist perceptions of the WA.

Conclusions: The WA emerged as a complex process that interacts with many factors and processes at play in the rehabilitation environment. Notwithstanding the limitations of the research base, findings indicate that enhancement of the WA may indeed influence rehabilitation outcomes. Allowing time for the development of the WA, and consideration of factors, such as therapist skill, may support therapists to strengthen their alliances in ABI rehabilitation.

\section{Luria Award for Most Outstanding Doctoral Student 2018}

\section{The Importance of Staying Connected: Mediating and Moderating Effects of Social Groups on Psychological Wellbeing After Brain Tumour}

Lee Cubis, ${ }^{1,2}$ Tamara Ownsworth,, ${ }^{1,2}$ Mark Pinkham, ${ }^{3,4}$ Melissa Legg, ${ }^{2,5}$ Matthew Foote, ${ }^{3,4}$ and Suzanne Chambers ${ }^{1,2,5}$

${ }^{1}$ School of Applied Psychology, Griffith University, Mount Gravatt, Australia

${ }^{2}$ Menzies Health Institute Queensland, Griffith University, Gold Coast, Australia

${ }^{3}$ School of Medicine, University of Queensland, Brisbane, Australia

${ }^{4}$ Department of Radiation Oncology, Princess Alexandra Hospital, Woolloongabba, Australia

${ }^{5}$ Cancer Council Queensland, Fortitude Valley, Australia

$\boldsymbol{R}$ ackground and objectives: Functional impairments contribute to psychological distress after brain tumour. Changes in social groups and confidence in support potentially account for, or alter this relationship. This study aimed to investigate the influence of social groups on functional impairment and psychological wellbeing. 
Method: 70 people with primary brain tumour (46\% benign; $18 \%$ low grade and 36\% high grade) aged 22-75 years completed measures of cognitive and physical impairment (Functional Assessment of Cancer Therapy), social groups (Exeter Identity Transition Scale), confidence in social support (Self-Efficacy Scale), depression, anxiety and life satisfaction. A bootstrapping method and PROCESS macro was utilised to test mediating and moderating effects of social group variables, controlling for relevant demographic and tumour characteristics.

Results: The relationship between cognitive and physical impairment and psychological distress was significantly mediated by maintenance of social groups. Indirect effects were found for depression (CI: 0.019-0.109), anxiety (CI: 0.013-0.064) and life satisfaction (CI: $-0.094 \sim-0.013)$. Forming new social groups had a moderating effect for life satisfaction $(p<.05)$, whereby individuals perceiving more cognitive impairment who were better able to develop new social groups reported higher life satisfaction. Confidence in social support was a moderator for depression $(p<.001)$, such that those perceiving more physical impairment who were more confident in their social support reported lower depression.

Conclusions: Functional impairment is related to increased psychological distress through loss of social groups. However, development of new social groups and greater confidence in support can buffer the effects of functional impairment on psychological wellbeing after brain tumour.

\title{
Travel Award for Students 2018
}

\section{Getting on the Same Wavelength: Clinicians' Perspectives of the Therapeutic Alliance in Community Brain Injury Rehabilitation}

\author{
Liz Williams, ${ }^{1,2}$ Jacinta Douglas, ${ }^{1,3}$ and Tanya Serry ${ }^{1}$ \\ ${ }^{1}$ College of Science, Health and Engineering, School of Allied Health; Department of Community \\ and Clinical Allied Health; Living with Disability Research Centre, Melbourne (Bundoora), Australia \\ ${ }^{2}$ Brain Injury Rehabilitation Community and Home program (BIRCH) SA Brain Injury Rehabilitation \\ Service (SA BIRS), Hampstead Rehabilitation Centre, Australia \\ ${ }^{3}$ Summer Foundation, Australia
}

$B$ ackground and aims: A positive therapeutic alliance has been associated with superior outcomes in the psychotherapeutic literature, and more recently in the acquired brain injury (ABI) rehabilitation literature. The aim of this study was to explore how clinicians experience the therapeutic alliance when working together with individuals with $\mathrm{ABI}$ and their significant others during community rehabilitation.

Method: A constructivist, qualitative methodology using grounded theory analysis techniques was applied. Participants were 12 clinicians across disciplines from a community ABI rehabilitation service. In-depth interviews were conducted with each participant and transcribed verbatim. Transcripts were progressively analysed using an iterative process of constant comparison.

Results: All participants highly valued the therapeutic alliance and data analysis revealed multiple themes active within the process of building and supporting the alliance. Themes captured the values that therapists used to guide the therapeutic process; coming to know and connecting with individuals; working together in partnerships and teamwork; and facilitators and barriers to building and maintaining alliances. These findings are congruent with those 
of an earlier study exploring the perspectives of individuals with brain injury and their significant others who attended the same rehabilitation service.

Conclusions: Clinicians considered the therapeutic alliance to be a pivotal aspect in the work of rehabilitation that afforded benefits for people with ABI and their significant others. Further research is needed to understand how the alliance and its key components shape outcomes in brain injury rehabilitation and to explore potential staff training approaches to optimise its development and maintenance.

Mindlink Brightwater Award for Interdisciplinary Research 2018

\title{
The Building Bridges Project: Linking Disconnected Service Networks in Acquired Brain Injury and Criminal Justice
}

\author{
Glenn Kelly, ${ }^{1}$ Suzanne Brown, ${ }^{2}$ and Grahame K. Simpson ${ }^{3,4}$ \\ ${ }^{1}$ Private practice, Melbourne Australia \\ ${ }^{2}$ Concept Psychology Services, Melbourne Australia \\ ${ }^{3}$ Brain Injury Rehabilitation Research Group, Ingham Institute of Applied Medical Research, Sydney \\ Australia \\ ${ }^{4}$ John Walsh Centre for Rehabilitation Research, University of Sydney, Sydney Australia
}

$\boldsymbol{R}$ ackground and aims: An evaluation was conducted of the Building B Bridges Project, a novel professional development model designed to increase cross-sectoral knowledge and collaboration between the acquired brain injury (ABI) and criminal justice (CJ) sectors.

Method: A total of 178 service providers from across the state of Victoria, Australia, participated in six professional development forums. The forum content spanned information about ABI, policing, disability legal rights and supports, and correctional/post-release services. Participants came from the disability, criminal justice, and health and community service sectors. Using a pretest-posttest design with six-month follow up, data were obtained via a project-specific questionnaire evaluating knowledge and behaviour in addressing $\mathrm{ABI} / \mathrm{CJ}$ issues.

Results: Statistically significant gains in knowledge were shown among participants at post-test, which were also maintained at follow up. At six-month follow up, work-related behaviours addressing ABI/CJ issues had increased significantly compared to the six months prior to the forum. Participants also reported high levels of satisfaction with the forums.

Conclusions: Carefully constructed professional forums improved cross-silo collaboration in the $\mathrm{ABI} / \mathrm{CJ}$ sectors. The project illustrates effective use of existing disability and justice resources, and highlights the importance of training approaches as part of a raft of initiatives needed to address the overrepresentation of people with $\mathrm{ABI}$ in the $\mathrm{CJ}$ system. 
Workshop 1

\title{
Rehabilitation as a Social Imperative: Developing Meaning and Purpose and a Reason to Get Out of Bed in the Morning
}

Tim Feeney

Belevedere Health Services, Albany, NY, USA

\begin{abstract}
$W$ hy am I getting out of bed? For many people with brain injury, there is no satisfying answer to this question. As a result, life post injury - for individuals and families and friends alike - can be dominated by frustration, confusion, withdrawal, anger and sadness. With these realities in mind, the proposition of this presentation is simple: All rehabilitation is fundamentally a quest to help people with brain injury find a reason to get out of bed each and every morning.

Essential to this quest are supports that are founded on the development of powerful and positive personal and social identities. It encompasses a flexible framework of support, with an emphasis on the inclusion of interventions that produce outcomes valued by the individual, are non-disability oriented, and embrace supports that are designed to assist in the growth of social competence. All of the approaches represented in this framework are built on a foundation of optimism and hope, with the guiding principles that we can always find something that works, that difficulties are expected and we can learn from each period of difficulty and make adjustments as needed, and that when we learn to collaborate with each other with good will we can achieve lasting positive outcomes, which will result in a lifetime full of reasons to get out of bed. For everyone!

After this workshop delegates should

- Understand a person-directed framework for rehabilitation that focuses on the development of positive identity and meaningful engagement;

- Understand the importance of developing supports that are proactive and founded on positive changes - with little import placed on diagnosis and disability; and

- Develop a framework for programme development with a focus on project-based interventions designed to support individuals with brain injury to become important contributors to their communities.
\end{abstract}

\section{Workshop 2}

\section{Understanding and Building Family Resilience After Traumatic Brain Injury}

Grahame Simpson

Brain Injury Rehabilitation Research Group, Ingham Institute of Applied Medical Research

ver the past three decades, a large number of studies have investigated the prevalence and risk factors of psychological distress and caregiver burden among family members supporting relatives with traumatic brain injury (TBI). The last five years have seen the beginning of a paradigm shift towards investigating the applicability of strength-based approaches to the conceptualisation and prediction of family adaptation after TBI. A core construct within 
strength-based approaches is resilience. The potential application of this construct to the field of neurorehabilitation is undergirded by research among other clinical populations, which has found that resilience has been associated with reduced levels of morbidity in the face of trauma or chronic stressors. The workshop will outline contextual factors underpinning the emergence of resilience research in neurorehabilitation; approaches to defining resilience; the application of resilience theory to traumatic injury; the broadening of the construct to encompass family resilience; current research in understanding family resilience after TBI; and early interventions to build resilience among families supporting relatives with TBI.

After this workshop, you should be able to

- Explain why resilience is an important framework in rehabilitation and health care.

- Identify different theoretical approaches to the conceptualisation of resilience.

- Understand the role of resilience in family adaption to traumatic brain injury.

- Demonstrate ability to recognise strengths in families.

Workshop 3

\section{Working Alongside People Following Brain Injury: Exploring Human Connection and Sense of Wellbeing When Taking a Lifeworld Approach to Service Provision}

Caroline Ellis-Hill

Bournemouth University, Bournemouth, UK

\footnotetext{
$A$ lifeworld approach privileges subjective experience and what life feels like from the inside out. Often in Acquired Brain Injury (ABI) services we, as clinicians, are looking and gaining knowledge from the outside using our intellectual abilities (head) to work out how to help people living with ABI. This can lead to separation between service users and service providers and can make it difficult for wellbeing to flourish. Using a lifeworld approach we use an inside perspective and use knowledge from our head (intellect), heart (embodied knowing) and hand (practical knowing) to create connections and allow a fully human response, which will support wellbeing.

In this very practical workshop, we will

- Explore what lifeworld means from our own experience.

- Explore how we can gain access to our embodied resources.

- Consider how we can meet others in a fully human way in our ABI practice.

Develop a plan for how you may want to introduce a lifeworld approach into your own clinical or research practice
} 
Workshop 4

\section{Co-Creating Health: Supporting People to Live Well in the Context of a Neurological Condition}

Nicola Kayes

University of Auckland, Auckland, New Zealand

$\mathrm{n}$ the context of a health system with limited resources, positive long-term
outcomes for people living with neurological impairment rely heavily on those individuals engaging in self-management activities that improve physical and emotional health and wellbeing. However, the physical, emotional, cognitive and behavioural consequences of neurological injury or illness frequently have a detrimental impact on one's ability to activate the regulatory processes necessary for successful self-management. Further, existing rehabilitation processes may inadvertently limit the potential for long-term health and wellness.

In this workshop, we will

a) look critically at existing processes;

b) explore how we could work differently at the point of care to support people to live well; and

c) discuss what it would take to embed strategies into practice to cocreate health for people living with the enduring impact of neurological impairment.

\section{ABSTRACTS - FRIDAY 4th MAY}

Plenary 1

\section{Listen to the story, not the statistic}

Caleb Rixon

$\Gamma \mathrm{n}$ this presentation, I share my experiences as a stroke survivor - to illu1 minate the perspectives of a person at the heart of a neurological trauma. I also talk about the impact of language and communications, and ways we can work together to create opportunities for connection.

\section{What Exactly is Evidence-Based Practice? Finding Evidence in Our Day to Day Work to Support Our Work Everyday}

Tim Feeney

Belvedere Health Services, Albany, NY, USA

$F$ vidence-based practice is a great! Necessary! Critical! But what consti$C$ tutes 'evidence'? The peer-reviewed research reports of the results of randomised controlled trials? The generational wisdom informing the 'models' of rehabilitation used in my workplace? The latest neuroscientific literature explaining interesting findings of increasingly sensitive brain scans? Yes. These should inform our clinical decision-making. And no. The contexts in which we do our work are rarely constant, the people with whom we work are 
complicated and don't fit neatly into a model of practice, and we don't often have the luxury of knowing exactly what the heck is going on in the brains of the people we support. As people who work in the messy real world, we need understand the bigger world of evidence, but more importantly, use our skills and talents to create our own evidence-based practices. With this in mind, the goal of this presentation is to create a foundation to integrate available research into our clinical practice while using the data from our everyday work to establish an evidentiary basis to demonstrate the effectiveness and validity of the work that we do.

\section{Session 1: Understanding and meeting emotional needs}

\section{The Interaction of Psychological and Cognitive Flexibility After a Traumatic Brain Injury}

Diane L. Whiting, ${ }^{1,2}$ Grahame K. Simpson, ${ }^{1,3}$ and Kasey Metcalf ${ }^{1,4}$

${ }^{1}$ Brain Injury Rehabilitation Research Group, Ingham Institute of Applied Medical Research, Liverpool, Australia

${ }^{2}$ Australasian Rehabilitation Outcomes Centre, Australian Health Services Research Institute, University of Wollongong, Australia

${ }^{3}$ John Walsh Centre for Rehabilitation Research, University of Sydney, Australia

${ }^{4}$ Brain Injury Rehabilitation Unit, Liverpool Hospital, Australia

$\boldsymbol{B}$ ackground and aims: A lack of psychological flexibility has been linked $B$ to a wide range of mental health problems and is proposed to be the mechanism for change in Acceptance and Commitment Therapy (ACT). Psychological flexibility is proposed to be associated with cognitive flexibility but this has not been empirically established. Psychological and cognitive flexibility were measured in individuals with a traumatic brain injury (TBI), who typically demonstrate impairments in their cognitive flexibility, to explore the relationship between the constructs.

Method: A total of 73 participants with a moderate to severe TBI were recruited from Liverpool Brain Injury Rehabilitation Unit. They were administered self-report measures of psychological flexibility (using measures of acceptance and action: Acceptance and Action Questionnaire-II (AAQ-II) \& Acceptance and Action Questionnaire - Acquired Brain Injury 9AAQ-ABI)) in conjunction with a full neuropsychological assessment.

Results: Functional measures of cognitive flexibility that assess the 'ability to shift' were not related to psychological flexibility but broader measures of cognitive flexibility that capture additional cognitive processes, were correlated. This relationship became non-significant when general intelligence was controlled in most measures of cognitive flexibility with the exception of verbal generativity and verbal inhibition.

Conclusions: Components of cognitive flexibility, namely verbal generativity and verbal inhibition, are significantly related to psychological flexibility in individuals with a TBI. This suggests an overlap between the constructs of cognitive flexibility and psychological flexibility within this population. 


\title{
The Association Between Psychological Resilience and Participation Outcomes Following Mild to Severe Traumatic Brain Injury
}

\author{
Carla Wardlaw, ${ }^{1}$ Amelia J. Hicks, ${ }^{2}$ and Jennie L. Ponsford ${ }^{2}$ \\ ${ }^{1}$ Monash University, Clayton, Australia \\ ${ }^{2}$ Monash-Epworth Rehabilitation Research Centre, Monash Institute of Cognitive and Clinical Neu- \\ rosciences, School of Psychological Sciences, Monash University, Melbourne, Australia
}

\begin{abstract}
$B$ ackground and aims: Traumatic brain injury (TBI) causes physical and cognitive-behavioural impairments that reduce participation in employment, leisure and social relationships. Given the impact on quality of life, enhancing understanding of factors associated with participation may inform rehabilitation to improve outcomes. Previous studies demonstrate that demographic and injury-related factors account for a relatively small proportion of variance in participation post-injury. Recent studies suggest that personal factors such as resilience may also impact outcomes. This study aimed to examine the association of resilience alongside demographic, injury-related, cognitive, emotional and family factors with participation following TBI.

Methods: A subset of results from the Longitudinal Head Injury Outcome Study undertaken by the Monash-Epworth Rehabilitation Research Centre were analysed. Resilience was measured using the Traumatic Brain InjuryQoL (TBI-QoL) Resilience Scale. Participation, covering productivity, leisure and social relations were measured using the Participation Assessment with Recombined Tools-Objective (PARTO-O-17). Data from 245 participants (73.5\% males; $M_{\text {age }}=44.41, S D_{\text {age }}=16.09 ; 94.7 \%$ moderate-to-severe TBI) was analysed. Multiple regression was used to assess the extent to which resilience, along with demographic, injury and post-injury variables predicted participation outcomes. Mediation analyses examined the impact of HADS anxiety and depression on the relationship between resilience and participation outcomes. Results: Resilience was a significant predictor of higher participation, along with lower injury severity, more years since injury, higher education and IQ, and younger age. Depression mediated the relationship between resilience and participation; however, anxiety was not a significant mediator of this relationship.

Conclusions: Further research investigating whether resilience-enhancing interventions may help reduce symptoms of depression and enhance participation following TBI is warranted.
\end{abstract}

\section{The 'I think part of going through the accident is, the challenge to your own identity': Thoughts About Identity Arising From Students' Participation in Education Following Traumatic Brain Injury}

\author{
Margaret Mealings, ${ }^{1,2}$ Jacinta Douglas, ${ }^{1,3}$ and John Olver ${ }^{2,4}$ \\ ${ }^{1}$ School of Allied Health, La Trobe University, Bundoora, Australia \\ ${ }^{2}$ Epworth Rehabilitation, Richmond, Australia \\ ${ }^{3}$ Summer Foundation, Box Hill, Australia \\ ${ }^{4}$ Epworth Monash Rehabilitation Medicine Unit, Richmond, Australia
}

$\boldsymbol{R}$ ackground and Objectives: Following Traumatic Brain Injury (TBI), participating in education often provides a pathway for returning to important aspects of a student's pre-injury life and identity. However, the process of successfully managing study is not easy for students given the potential 
impacts of wide spread TBI difficulties. When the ability to participate in study is affected, how do students respond to the challenge to their pre-injury identity? In this paper, we explore themes related to identity that emerged from students' reflections about their study journey.

Method: The data for this project are drawn from a longitudinal, mixed method investigation. 12 students (17-32 years) completed in-depth interviews and a well-being questionnaire up to three times over a period of 4-15 months. A total of 30 time points were captured. Data were analysed using grounded theory methods and descriptive statistics.

Results: Three themes related to identity emerged: 'Me', the way students described their pre-injury self-concept; 'TBI', perspectives of how the TBI had affected students; and, 'Is it me or TBI?', an area of overlap and tension between Me and TBI. This tension resulted in fluctuations in students' evaluations of wellbeing over time.

Conclusions: Participating in education following TBI can be seen as part of a larger process of return to life and identity rebuilding. The themes identified in this research show that clinicians and educators must adopt a holistic, flexible approach to supporting students that can be adapted to reflect the individual and dynamic processes involved.

\title{
Is Stepped Psychological Care the Solution? Perspectives of Stroke Health Professionals and People With Aphasia on Managing Mood and Depression
}

\author{
Caroline Baker, ${ }^{1}$ Linda Worrall, ${ }^{1}$ Miranda Rose, ${ }^{2}$ and Brooke Ryan ${ }^{1}$ \\ ${ }^{1}$ School of Health and Rehabilitation Sciences, The University of Queensland, Brisbane, Australia \\ ${ }^{2}$ School of Allied Health, La Trobe University, Melbourne, Australia
}

R ackground and aims: Aphasia, commonly caused by stroke affects the ability to talk, understand, read and write. Approximately $60 \%$ of people with aphasia also experience depression at 12 months post-stroke but there is a lack of psychological care. Stepped psychological care may potentially bridge the gap in services for people with aphasia. The aims of this research are to explore the perspectives of: (1) stroke health professionals on current practice and the barriers and facilitators to stepped psychological care and (2) people with aphasia on their experience of mood and/or depression and views of stepped psychological care.

Method: Two qualitative studies were conducted through (1) five focus groups with 39 stroke health professionals and (2) interviews with 10 people with aphasia. Verbatim transcripts were analysed using Interpretive Description.

Results: Themes of current practice included an overarching theme that aphasia and mood is a challenging area of practice. Barriers exist, however ways to overcome these were reported, such as skill training. People with aphasia conveyed experiences, such as trauma at onset of stroke and aphasia. They viewed communication, various supports and activities as important in a stepped care approach.

Conclusions: This research challenges stroke health professionals and researchers to develop innovative ways to overcome the challenges of supporting mood change and depression in people with aphasia. Future research is warranted to explore strategies to implement components of stepped care, such as training for stroke health professionals and the effectiveness of interventions to target depression outcomes for people with aphasia. 


\title{
A Shared Journey: Utilising Peer Support to Empower People With Acquired Brain Injury to Live Their Best Lives via Meaningful, Valued Roles
}

\author{
Jennifer L. Farnden ${ }^{1,2}$ \\ ${ }^{7}$ Families4Families Inc, Adelaide, Australia \\ 2 Jennifer Farnden, Consulting Services, Australia
}

\begin{abstract}
$B$ ackground and aims: Families4Families provides information and education sessions, social opportunities, on-line resources and other family centric supports via peer-based programmes they have delivered across SA since 2012. Our new research project focuses on improving the participation of people with ABI across our communities via empowering them to gain meaningful and valued roles. Supported by the Julia Farr Association via their MS McLeod Benevolent Fund, Families4Families is currently undertaking this study into the ways in which people living with Acquired Brain Injury can utilise peer support principles to build their community participation via gaining and retaining valued roles.

This 20 month project (June 2017-January 2019) will enable Families4Families to document the key processes most successful in bringing about participation for the future benefit of people living with ABI nationally.

Method: A case-based design is being utilised in which 15-18 participants with severe $\mathrm{ABI}$ are provided with a range of peer support interventions over a 12 month process focused on improving their participation via self-identified valued roles. Pre- and Post-survey tools will assess changes in roles and community integration.

Results: Participants will be part way through the intervention at the time of the conference, and initial pre-survey evidence will be presented along with early evidence and case stories.

Conclusions: In this presentation, background evidence will illustrate the need for the study due to the lack of valued role opportunities for people living with severe $\mathrm{ABI}$ in our target audience, and early evidence from the project will also be presented.
\end{abstract}

\section{Predicting Mood Outcome After Traumatic Brain Injury (TBI) Using the Hospital Anxiety \& Depression Scale (HADS) Factors}

\author{
Matthew Thomas, ${ }^{1}$ Clive Skilbeck, ${ }^{2}$ Kieran Holm, ${ }^{2}$ and Mark Slatyer ${ }^{2}$ \\ ${ }^{1}$ School of Psychology, Charles Sturt University, Bathurst, Australia \\ ${ }^{2}$ School of Medicine (Psychology), University of Tasmania, Hobart, Australia
}

R ackground and objectives: The Hospital Anxiety and Depression Scale $B$ (HADS) is frequently used to assess mood following Traumatic Brain Injury (TBI). Research has identified a three-factor structure (anxiety, depression and psychomotor) as useful in measuring mood dysfunction post-TBI. The study's objective was to identify demographic, clinical and variables obtained at one-month assessment to predict HADS factor scores up to 24 months post-injury.

Method: Data from a sample of 596 participants, were analysed using Multiple Regression (MR) to identify significant predictor variables.

Results: At each follow-up MR equations were generated to predict factor scores, the variance accounted for ranging between 21-27\% (anxiety), 25$30 \%$ (depression), and 29-33\% (psychomotor). These increased to $47-55 \%$, 
$37-42 \%$ and $44-51 \%$, respectively, when HADS factor scores at one month post-injury were included. Estimated pre-morbid IQ (National Adult Reading Test) and post-concussion symptoms (Rivermead Post-Concussion Symptoms Questionnaire) were included in all equations for anxiety and psychomotor scores, and depression scores at six and 12 months post-TBI. Age and estimated pre-morbid quality of life (Quality of Life Inventory) were frequently included to predict depression and psychomotor scores, but not anxiety. Other variables (e.g., pain at one month, cause of TBI, gender and orthopaedic damage) were occasionally included in prediction equations.

Conclusions: HADS factor scores up to two years after TBI can be significantly predicted from a combination of demographic and clinical variables. There is sufficient variation in the identified predictor variables to support a three-factor HADS.

Session 2: Treatment and outcome considerations in rehabilitation

\section{Population-Based, Longitudinal Cohort Study of Symptoms and Participation in Adults Four Years Following a Mild Traumatic Brain Injury}

Alice Theadom, ${ }^{1}$ Nicola Starkey, ${ }^{2}$ Suzanne Barker-Collo, ${ }^{3}$ Kelly Jones, ${ }^{1}$ Shanthi Ameratunga, ${ }^{4}$ and Valery Feigin, ${ }^{1}$ on behalf of the BIONIC Research Group

$\boldsymbol{B}$ ackground and aims: Nearly half of adults sustaining a mild trauDatic brain injury (TBI) experience persistent post-concussion symptoms. However, it remains unclear as to how long these symptoms may persist and if they have any longer-term implications. This study aimed to identify if any deficits are evident four years following a mild TBI.

Method: From an incidence, cohort of 341 adult participants assessed 12months post-injury, $232(68.0 \%)$ completed a follow-up assessment four years post-injury. Outcomes were compared with age and sex matched controls $(N=232)$. Participants provided socio-demographic information and completed the Rivermead Post-Concussion Symptom Questionnaire and the Participation Assessment with Recombined Tools questionnaire.

Results: The mild TBI sample experienced significantly increased cognitive symptoms $(t=2.17, p=.03)$ four years post-injury in comparison to controls. However, there were no differences between the groups for somatic $(t=-1.07$, $p=.28)$ or emotional symptoms $(t=-1.60, p=.11)$. There was no significant change in total symptom scores between one $(\bar{x}=16.35, S D$ 14.04) and four years post-injury $(\bar{x}=16.54, S D 12.93, t=-0.39, p=.70)$. Additionally, the mild TBI group reported significantly poorer community participation at four years in comparison to controls across all three domains: productivity $(t=-13.87, p<.01)$, social relations $(t=-3.77, p<.01)$ and getting out and about $(t=-16.89, p<.01)$.

Conclusions: Long-term impacts of TBI may include persistent cognitive symptoms and difficulties in terms of productivity, social relationships and ability to participate in everyday activities. These findings support the need for early intervention following mild-TBI for those who do not experience spontaneous recovery to reduce the potential longer-term impacts. 


\title{
Patient Experiences of Occupational Therapy Groups in Traumatic Brain Injury Rehabilitation
}

\author{
Freyr Patterson, ${ }^{1}$ Jennifer Fleming, ${ }^{1,2}$ and Emmah Doig ${ }^{1}$ \\ ${ }^{1}$ School of Health and Rehabilitation Sciences, The University of Queensland, Brisbane Australia \\ ${ }^{2}$ Occupational Therapy Department, Princess Alexandra Hospital, Brisbane, Australia
}

\begin{abstract}
$\boldsymbol{R}$ ackground and objectives: Currently, there is limited evidence to guide clinicians in the planning and delivery of groups to patients following Traumatic Brain Injury (TBI). There is also a paucity of research that captures patient experiences of participating in group interventions. Seeking patient feedback is one way to explore their experiences, and to engage consumers in service development, delivery and evaluation.

The aims of the study were to explore the perceptions and experiences of people with TBI about their participation in inpatient occupational therapy rehabilitation groups.

Method: A phenomenological approach guided the study. Patients with a TBI who were participating in an inpatient rehabilitation group's programme were recruited. Data were collected through a series of semi-structured interviews, and analysed using content analysis.

Results: Fifteen participants consented to participate. Three main themes emerged, feeling normal, comfort and connected; learning by doing, seeing and sharing and; practicalities of groups and recommendations.

Conclusions: Groups can facilitate opportunities for peers to engage with, and learn from each other in ways that individual therapy sessions cannot. Groups can also provide an ideal environment and opportunities for learning to occur, and facilitate a sense of normalcy for participants. The findings from this study support the use of occupational therapy groups as a therapeutic intervention in TBI rehabilitation. Recommendations from the perspectives of patients for clinical practice will be presented.
\end{abstract}

\section{Online Education and Cognitive-Behavioural Interventions to Improve Sleep Quality After Traumatic Brain Injury: A Pilot Study}

Alice Theadom, ${ }^{1}$ Suzanne Barker-Collo, ${ }^{2}$ Kelly Jones, ${ }^{1}$ Margaret Dudley, ${ }^{2}$ Norah Vincent, ${ }^{3}$ and Valery Feigin ${ }^{1}$

${ }^{1}$ National Institute for Stroke and Applied Neuroscience, Auckland University of Technology, Auckland, New Zealand

${ }^{2}$ School of Psychology, University of Auckland, Auckland, New Zealand

${ }^{3}$ Department of Clinical Health Psychology, University of Manitoba, Winnipeg, Canada

$\boldsymbol{R}$ ackground and aims: Poor sleep quality is one of the most common consequences of traumatic brain injury (TBI). This study aimed to explore the feasibility and potential efficacy of on-line interventions to improve sleep quality post-TBI.

Method: Twenty-four participants $(N=9$ male, $N=15$ female, mean age $35.9 \pm 11.8$ years) who reported experiencing sleep difficulties between 3-36 months after a mild or moderate TBI, were randomised to receive either a cognitive behaviour therapy (CBT, $N=12)$ or an education intervention $(N=12)$ on-line. Both interventions were delivered for 20-30 minutes per week, over a six-week period. Outcome assessments conducted pre- and post-intervention included the Pittsburgh Sleep Quality Index, actigraphy the CNS Vital Signs on-line neuropsychological test, and the Rivermead Post-concussion Symptoms and Quality of Life after Brain Injury questionnaires. 
Results: Both programmes demonstrated feasibility for use post-TBI, with $83.3 \%$ of participants completing the interventions. Participants used the interventions on average twice weekly for an average duration of 10.9 minutes. The CBT group experienced significant improvement $(F=5.47, p=0.04)$, in overall self-reported sleep quality in comparison to controls post-intervention, with a moderate effect size of 1.17. There were no significant group differences on objective sleep quality domains, neuropsychological functioning, post-concussion symptoms or quality of life.

Conclusions: On-line programmes designed to improve sleep are feasible for use for adults following mild to moderate TBI. Full-scale clinical trials are needed to determine clinical effectiveness of on-line interventions for people post-TBI.

\title{
Efficacy of Melatonin Supplementation for Sleep Disturbances Following Traumatic Brain Injury: Findings from a Randomized Controlled Trial
}

\author{
Natalie A. Grima, ${ }^{1,2,3}$ Shantha M.W. Rajaratnam, ${ }^{4,5,6}$ Darren Mansfield,,7 Tracey L. \\ Sletten, ${ }^{4}$ Gershon Spitz, ${ }^{1,2,4}$ and Jennie L. Ponsford ${ }^{1,2,4}$ \\ ${ }^{7}$ Monash-Epworth Rehabilitation Research Centre, Australia \\ ${ }^{2}$ Epworth Hospital, Australia \\ ${ }^{3}$ Department of Psychiatry, Harvard Medical School, USA \\ ${ }^{4}$ Monash Institute of Cognitive and Clinical Neurosciences, School of Psychological Sciences, Aus- \\ tralia \\ ${ }^{5}$ Division of Sleep and Circadian Disorders, Departments of Medicine and Neurology, Brigham and \\ Women's Hospital and Harvard Medical School, USA \\ ${ }^{6}$ Division of Sleep Medicine, Department of Medicine, Harvard Medical School, USA \\ ${ }^{7}$ Monash Lung and Sleep, Monash Health, Australia
}

$\boldsymbol{R}$ ackground and aims: Sleep disturbances commonly occur following B Traumatic Brain Injury (TBI); however, evidence-based treatments are lacking. The aim of this study was to determine the effectiveness and safety of melatonin supplementation for sleep disturbances in patients with TBI.

Method: Randomised, double-blind, placebo-controlled, two-period, twotreatment (melatonin and placebo) crossover study. Outpatients were recruited from Epworth and Austin Hospitals Melbourne, Australia. Outpatients with mild to severe TBI $(n=33)$ reporting sleep disturbances post injury (mean age $37(S D, 11)$ years; $67 \%$ men). Prolonged-release melatonin formulation $\left(2 \mathrm{mg}\right.$; Circadin $\left.{ }^{\mathrm{R}}\right)$ and placebo capsules for 4 weeks each in a counter-balanced fashion.

Results: Melatonin supplementation significantly reduced global Pittsburgh Sleep Quality Index scores relative to placebo, indicating improved sleep quality (melatonin 7.68, vs. placebo 9.47, original score units; difference-1.79; $95 \%$ CI -2.70 to $-0.89 ; p \leq .0001)$. Melatonin had no effect on sleep onset latency (melatonin 1.37, vs. placebo 1.42, log units; difference -0.05 ; $95 \%$ $\mathrm{CI}-0.14$ to $0.03 ; p=.23$ ). Across secondary outcome measures, melatonin increased sleep efficiency, vitality and mental health. Melatonin also decreased anxiety and fatigue.

Conclusions: Melatonin supplementation over a 4- week period is effective and safe in improving subjective sleep quality as well as some aspects of objective sleep quality in patients with TBI. 


\title{
The Benefits of Neuropsychological Assessment Feedback Vary According to Patients' Level of Cognitive Impairment: Results From a Randomised Controlled Trial With Cross-Over in Multiple Sclerosis
}

\author{
Wendy Longley, ${ }^{1}$ Robyn Tate, ${ }^{1}$ and Rhonda Brown ${ }^{2}$ \\ ${ }^{1}$ John Walsh Centre for Rehabilitation Research, Kolling Institute of Medical Research, University of \\ Sydney, Sydney, Australia \\ ${ }^{2}$ Research School of Psychology, Australian National University, Canberra, Australia
}

\begin{abstract}
$\boldsymbol{B}$ ackground and aims: There is no strong evidence supporting the clinical 3 efficacy of providing neuropsychological assessment (NPA) feedback to patients or caregivers. This randomised controlled trial with cross-over investigated the therapeutic benefits of NPA feedback in multiple sclerosis (MS) using a semi-structured, collaborative feedback protocol delivered by clinical neuropsychologists experienced in MS.

Method: Participants were recruited from a Sydney community-based MS service, and randomly assigned to either the NPA feedback intervention or sham-waitlist control group. Participants were administered outcome evaluations by a research assistant blind to group allocation on four occasions one month apart; once prior to the NPA for the intervention group, and twice prior for the controls. A wide range of psychological outcome measures was utilised. Patients were divided into three subgroups according to neuropsychologists' ratings of cognitive impairment. Effect sizes (partial eta squared, Cohen's d) were the predominant means of interpreting data.

Results: Seventy-one patients and 53 caregivers participated. Thirty-six patients were randomly assigned to NPA feedback intervention and 35 to control. Different cognitive subgroups improved on different measures of mood, perception of cognitive impairment, self-efficacy, strategy use, quality of relationship and caregiving outcomes at different times following NPA feedback. The No Abnormalities Detected subgroup showed early benefits, whereas the Mild and Major impairment subgroups took up to 9 weeks.

Conclusions: NPA feedback is a complex psycho-educational intervention and its therapeutic effect varies according to patients' level of cognitive impairment and the timing of outcome evaluation. Nevertheless, this RCT provides preliminary evidence for its clinical efficacy.
\end{abstract}

\section{The Value of Purpose Built Independent Living Units for People With an Acquired Brain Injury}

\author{
Janet Wagland, ${ }^{1}$ Elly Williams, ${ }^{1}$ and Angelita Martini ${ }^{1,2}$ \\ ${ }^{1}$ Brightwater Care Group, Perth, Australia, ${ }^{1}$ School of Population Health, University of Western \\ Australia
}

$\boldsymbol{R}$ ackground and objectives: Moving from post-acute rehabilitation to independent living accommodation following an acquired brain injury (ABI) can often lead to difficulties when people are not fully prepared. People are often faced with limited supports and are at high risk of failing to manage their finances, maintain and establish positive relationships and take care of their personal health. This study aimed to demonstrate the participation and independence gained by people with an ABI residing in purpose built independent living units attached to a post-acute rehabilitation facility before moving to separate unsupported accommodation. This study determined the skills learnt in independent living units that enabled them to successfully move into independent community accommodation. 
Method: Clients residing in $n=8$ independent living units attached to a post-acute rehabilitation facility. Pre and post: Mayo-Portland Adaptability Inventory-4 (MPAI-4) outcome measures were compared; and the level and type of staffing support was evaluated using Northwick Park Care Needs Assessment.

Results: Providing people with a supported independent living opportunity resulted in increased community participation, improved ability to manage money and improved self-care. Need for staff support decreased throughout individuals' stays in the ILUs.

Conclusions: To ensure successful and sustainable independent living following $\mathrm{ABI}$, it is important to consider supported independent living units at rehabilitation facilities. This will reduce the risk of failed discharge, harm (health and wellbeing) and homelessness by adequately preparing people for community living.

\section{Factors Influencing the Use of Smartwatch Technology for Symptom Self-Management}

Hannah Gullo, Tammy Aplin, Jessica Lim, and Javier Bara

School of Health and Rehabilitation Sciences, The University of Queensland, Brisbane, Australia

$\boldsymbol{R}$ ackground and objectives: Smartwatches, which synchronise with applications on personal mobile devices, provide an innovative means of delivering rehabilitation strategies, whilst enhancing self-management of symptoms associated with a chronic neurological condition. This study aimed to investigate user confidence and satisfaction in using smart technology for selfmanagement of symptoms associated with multiple sclerosis (MS). Specifically, to determine if demographic and disease variables were predictive of baseline confidence and satisfaction in smart technology use.

Method: This study involved eight community-dwelling adults with MS about to embark on a 4-week programme focused on self-management of cognitive and mood problems, and fatigue delivered by an occupational therapist. Participants completed a newly developed questionnaire, the Smart Technology Satisfaction and Confidence Scale (STSCS) at baseline to examine perceptions of satisfaction and confidence in using smart technology. Linear regressions were used to determine the predictive value of demographic and disease characteristics.

Results: Age, marital status, living situation, education, work status and disease severity were significantly related to baseline confidence and satisfaction ratings on the STSCS.

Conclusions: Smartwatches present a novel platform for assisting with selfmanagement of disabling symptoms for people living in the community. Exposure to the latest smart technology may improve user confidence. Training in its application should be tailored based on user demographics, disease severity, baseline confidence and aspects of usability. 
Session 3: Working with children

\title{
Life Altering Effects on Children When a Family Member has an Acquired Brain Injury; A Qualitative Exploration of Child and Family Perceptions
}

Kate Dawes, Ashley Belcher, and Maggie Killington

South Australian Brain Injury Rehabilitation Service, Royal Adelaide Hospital, Adelaide, Australia

\begin{abstract}
$\boldsymbol{R}$ ackground and aims: Literature indicates that children are negatively impacted by brain injury in the family and are at risk of developing emotional, relationship, somatic, developmental, psychopathology and problematic behavioural issues as a result. The aim of our research was to investigate the consumer perspectives of what the optimum support system should be; including what, how, when and where.

Method: Participants were recruited via three different streams of the South Australian Brain Injury Rehabilitation Service (inpatient, outpatient and ambulatory) so the sampling was diverse and covered different stages of recovery and impact amongst families. Participants included 20 children from two age categories (5-10 and 11-17) and 10 adults/significant others of children, who have experienced brain injury in the family. Qualitative interviews were performed and focused on involvement of children in rehabilitation, including their emotional, psychosocial and practical perspectives.

Results: Children and significant others were able to verbalise their feelings, actual experiences and provide insights into what support did and/or would have benefited their knowledge of brain injury and be a part of their loved ones recovery. Tailored age-appropriate resources need to be developed to assist Clinicians to actively involve and educate children.

Conclusions: No previous qualitative research has occurred with consumers, to get their perspectives and ideas on what resources or methods should be used when involving children in the rehabilitation of a significant person in their life with a brain injury. Our Research will be integral in the development of such resources, which will be the focus of the next phase of Research in 2018.
\end{abstract}

\section{Factor Analysis of the Paediatric Awareness Questionnaire for Children and Adolescents with Acquired Brain Injury: Preliminary Findings}

\author{
Owen Lloyd, ${ }^{1,2,3}$ Tamara Ownsworth, ${ }^{1}$ Jennifer Fleming, ${ }^{4,5}$ and Melanie Zimmer- \\ Gembeck $^{6}$ \\ ${ }^{1}$ School of Applied Psychology \& Menzies Health Institute of Queensland, Griffith University, Bris- \\ bane, Australia \\ ${ }^{2}$ Queensland Paediatric Rehabilitation Service, Lady Cilento Children's Hospital, Children's Health \\ Queensland, Brisbane, Australia \\ ${ }^{3}$ School of Psychology, University of Queensland, Brisbane, Australia \\ ${ }^{4}$ School of Health and Rehabilitation Sciences, University of Queensland, Brisbane, Australia \\ ${ }^{5}$ Occupational Therapy Department, Princess Alexandra Hospital, Brisbane, Australia \\ ${ }^{6}$ School of Applied Psychology \& Menzies Health Institute of Queensland, Griffith University, South- \\ port, Australia
}

$\boldsymbol{B}$ ackground and aims: There is preliminary evidence that children and adolescents with acquired brain injury (ABI) experience deficits in selfawareness, although there is a lack of validated tools to assess self-awareness in children. The Paediatric Awareness Questionnaire (PAQ) was developed to assess children's self-awareness in a range of functional areas typically 
affected by ABI. This study aimed to determine the underlying factor structure of the PAQ.

Methods: 154 children aged 8-16 years with acquired brain injury (63\% traumatic brain injury, $14 \%$ stroke, $12 \%$ tumour, $11 \%$ infection and $3 \%$ hypoxic) and their parents were recruited consecutively through the Queensland Paediatric Rehabilitation Service and completed the 37-item PAQ.

Results: One-way ANOVA indicated no significant differences in child, parent and parent-child discrepancy scores between the four main aetiology groups, supporting pooling of data for factor analysis. The items on the parent PAQ version were submitted to a principal components analysis with varimax rotation. Bartlett's test of sphericity was significant $(p<.001)$, with adequate sampling according to Kaiser-Meyer-Olkin (0.92). A five factor solution explained 62\% of the variance, with factors that reflect social-emotional functioning, physical functioning, cognitive abilities, activities of daily living, and communication. However, several items loaded on more than one factor.

Conclusions: The findings indicate that the parent version of the PAQ assesses several distinct functional domains. Further research is needed to determine whether children's self-awareness varies according to functional domains after ABI.

\title{
The Pediatric Evaluation of Emotions Relationships and Socialisation (PEERS): An Objective, Standardised Assessment of Social Competency
}

\author{
Simone Darling, ${ }^{1}$ Miriam Beauchamp, ${ }^{2,3}$ Stephen Hearps, ${ }^{1}$ and Vicki Anderson, ${ }^{1,4,5}$ \\ Penny Cropper presenting \\ ${ }^{1}$ Clinical Sciences, Murdoch Childrens Research Institute, The Royal Children's Hospital, Parkville, \\ Victoria, Australia \\ ${ }^{2}$ Department of Psychology, University of Montreal \\ ${ }^{3}$ Ste-Justine Hospital Research Center, Montreal, Canada \\ ${ }^{4}$ Melbourne School of Psychological Sciences, University of Melbourne, Parkville, Victoria, Australia \\ ${ }^{5}$ Psychology Service, The Royal Childrens Hospital, Parkville, Australia
}

$\boldsymbol{R}$ ackground: Mature social cognition is necessary for functional social B skills such as developing and sustaining rewarding relationships. Social skills deficits are a primary symptom of many neurodevelopmental disorders, yet the assessment of these skills is unstandardised. The Pediatric Evaluation of Emotions Relationships and Socialization (PEERS) is a novel assessment tool designed to provide a standardised, developmentally appropriate, ecologically assessment of social cognition. In addition, the complimentary questionnaire (PEERS-Q) is designed to collect proxy information about the child's functional social outcomes.

Methods: 200 children aged 4-18 years with clinical diagnoses, where social deficits are a primary symptom completed PEERS along with an age appropriate assessment of Intelligence Quotient between June 2016 and December 2017. Parent report of medical and developmental history, and parent and teacher report of functional social behaviour (Strengths and Difficulties Questionnaire (SDQ), Social Skills Improvement Scale (SSIS) and PEER-Q) was also collected. Age- and sex-based standard scores were derived using data collected from typically developing controls in a large-scale standardisation study.

Results: Compared to norms, the clinical groups displayed relative weaknesses in various social domains as measured by PEERS. Compared to the SDQ and SSIS and consistent with what would be expected clinically, the PEERS-Q identified a larger number of children with deficits in social functioning. 
Conclusions: There PEERS package is a useful aid in the characterisation of social competency in paediatric populations with neurodevelopmental conditions, assisting with clinical decision-making and tailoring interventions. The utility of PEERS may be generalisable to other populations across the lifespan, such as those with neurodegenerative disorders. Further investigations are warranted.

\title{
Seizure-Related Hospital Admissions, Readmissions and Costs: Comparisons With Asthma and Diabetes in South Australia
}

\author{
Michelle Bellon, ${ }^{1}$ Christopher Barton, ${ }^{2}$ Nikki McCaffrey, ${ }^{3}$ and Claire Hutchinson ${ }^{4}$ \\ ${ }^{1}$ Disability and Community Inclusion, Flinders University \\ ${ }^{2}$ General Practice, Monash University, Melbourne, Australia \\ ${ }^{3}$ School of Health \& Social Development, Deakin University, Melbourne, Australia \\ ${ }^{4}$ School of Health Sciences, University of South Australia, Adelaide, Australia
}

\begin{abstract}
$\boldsymbol{B}$ ackground and objectives: Seizures are listed as an Ambulatory Care B Sensitive Condition (ACSC), where, in some cases, hospitalisation may be avoided with appropriate preventative and early management in primary care. We examined the frequencies, trends and financial costs of first and subsequent seizure-related hospital admissions in the adult and paediatric populations, with comparisons to bronchitis/asthma and diabetes admissions in South Australia between 2012 and 2014.
\end{abstract}

Methods: De-identified hospital separation data from five major public hospitals in metropolitan South Australia were analysed to determine the number of children and adults admitted for the following Australian Refined Diagnosis Related Groups: seizure related conditions; bronchitis/asthma; and diabetes. Additional data included length of hospital stay and type of admission. Demographic data were analysed to identify whether social determinants influence admission, and a macro-costing approach was then applied to calculate the financial costs to the healthcare system.

Results: Although epilepsy is less prevalent in the Australian population than other chronic conditions, this study reports higher rates of seizure-related hospital separations than diabetes $(649 / 100,000$ and 50/100,000, respectively). In particular, a higher proportion of the total number of separations was subsequent hospitalisations among both children and adults with seizures than either asthma or diabetes.

Conclusions: Following the lead of well-developed and resourced health promotion initiatives in asthma and diabetes, appropriate primary care, community education and seizure management services (including seizure clinics) should be targeted in an effort to reduce seizure related hospitalisations which may be avoidable, minimise costs to the health budget and maximise healthcare quality. 


\section{Clinical Implementation of Manualised Everyday Memory Group Training for Children}

Jennifer Papoutsis, ${ }^{1}$ Kath Bakker, ${ }^{1,2}$ Melinda Barker, ${ }^{1,2}$ Debbie Houston, ${ }^{1}$ Suncica (Sunny) Lah, ${ }^{3}$ and Ali Crichton ${ }^{1,2}$

${ }^{1}$ Victorian Paediatric Rehabilitation Service, Parkville/Clayton VIC, Australia

${ }^{2}$ Murdoch Childrens Research Institute, Parkville Victoria, Australia

${ }^{3}$ The University of Sydney \& ARC Centre of Excellence in Cognition and its Disorders, Sydney New South Wales, Australia

ackground and objectives: There is reasonable evidence for the efficacy
of memory strategies to support memory functioning in children with
acquired brain injury (ABI). However, a structured evidence-based clinical
rehabilitation programme for memory is yet to be implemented into clinical
paediatric rehabilitation practice, which is the aim of the current study.
Methods: Two groups of six adolescents with ABI (aged range 11-19
years) participated in a six-week group memory programme. The study up-
dated and modified a group programme originally developed and evaluated
to be effective in reducing everyday memory difficulties in children with
ABI by Ho, Epps, Parry, Poole \& Lah (2011). Parents attended a concurrent
group which provided psycho-education and memory strategies to help chil-
dren. The memory group was evaluated for (i) feasibility (service utilisation
and consumer satisfaction), (ii) dosage (attendance and homework comple-
tion) and (iii) efficacy in reducing everyday memory difficulties (child and
parent memory and metacognition questionnaires). A subgroup also reported
on quality of life (child and parent rated socio-emotional measures). Pre-and
post-questionnaires were completed by all participants.
Results: Feasibility was demonstrated through high satisfaction ratings. At-
tendance was high, however, homework completion was variable. Not all
children benefitted equally. Overall, goal achievement was indicative of mean-
ingful improvement in everyday memory pre- to post- intervention, providing
preliminary evidence for efficacy in a small sample.
Conclusions: A group memory training programme seems feasible within the
clinical setting, supported by high attendance and satisfaction. Family factors
and socio-emotional changes should be further investigated, particularly in the
parent component of the group. Further evaluation in a larger sample size is
required.

\section{Cognitive, Behavioural and Psychological Outcomes in Unoperated Metopic Synostosis}

Amanda J. Osborn, ${ }^{1}$ Rachel M. Roberts, ${ }^{1}$ Jane L. Mathias, ${ }^{1}$ Peter J. Anderson, ${ }^{2}$ and Walter J. Flapper ${ }^{2}$

${ }^{1}$ School of Psychology, University of Adelaide, Adelaide, Australia

${ }^{2}$ The Australian Craniofacial Unit, Women's and Children's Hospital, North Adelaide, Australia

$\boldsymbol{R}$ ackground and objectives: Metopic synostosis (MS), also referred to as 'trigonocephaly', involves a deformation of the frontal skull due to premature closure of the metopic suture. Cognitive, behavioural and psychological problems are common in children with MS, but few studies have examined outcomes in those who were treated conservatively (unoperated). Thus, the current meta-analysis synthesised research examining the cognitive/behavioural/psychological functioning of children with unoperated MS. Method: Data from 10 studies that examined these outcomes in unoperated children with MS were analysed. Hedges' $g\left(g_{\mathrm{w}}\right)$ effect sizes compared out- 
comes - assessed using both objective and subjective measures - of samples with unoperated MS to healthy controls/normative data. Where available, the prevalence of problems was calculated.

Results: Children with unoperated MS $(N=249)$ performed significantly worse than their healthy peers on all domains that were examined, namely: general cognition $\left(g_{\mathrm{w}}=-.38\right)$, motor functioning, $\left(g_{\mathrm{w}}=-.81\right)$, verbal $\left(g_{\mathrm{w}}=\right.$ $-.82)$ and visuospatial $\left(g_{\mathrm{w}}=-.92\right)$ abilities. In addition, objective assessments revealed a high prevalence of problems in the areas of: general cognition $(18 \%)$, motor functioning (36\%), verbal abilities (75\%), global developmental delay $(96 \%)$ and behaviour (36\%). Moreover, motor (19\%) and behavioural (57\%) problems, autistic tendencies (76\%) and psychological difficulties (40\%) were also reported (subjective measures) frequently.

Conclusions: The cognitive, behavioural and psychological outcomes of children with unoperated MS are generally worse than their healthy peers. However, research is sparse, samples small and controls rarely recruited. Nevertheless, the findings suggest that children with unoperated MS are likely to experience a variety of negative outcomes and should therefore receive ongoing monitoring and support.

\title{
Vocabulary Skills of School-Age Children With Acquired Brain Injury: An Exploration of Tiered Word Knowledge and Naming Errors
}

\author{
Ruei Ern Chea, ${ }^{1}$ Kimberley Docking ${ }^{1}$ Natalie Munro, ${ }^{1}$ Suzi Drevensek, ${ }^{2}$ and Can- \\ dice Brady ${ }^{2}$ \\ ${ }^{1}$ Discipline of Speech Pathology, The University of Sydney, Sydney, Australia \\ ${ }^{2}$ Brain Injury Service, Kids Rehab, The Children's Hospital at Westmead, Sydney, Australia
}

$\boldsymbol{R}$ ackground and aims: Vocabulary deficits are among the most commonly 3 reported language difficulties following childhood acquired brain injury (ABI). Using a three-tiered framework, vocabulary can be categorised into tier one (basic words), tier two (high-frequency words used across contexts), and tier three (curriculum-based words) (Beck, McKeown, \& Kucan, 2013). This study examined the tiered vocabulary skills and naming error patterns in children with ABI.

Method: Eleven children (7;6-11;11), with moderate/severe ABI (>12 months post-injury), were individually age- and gender-matched with typically developing (TD) controls. Receptive and expressive tests of the Australian version of the Montgomery Assessment of Vocabulary Acquisition (Montgomery, 2008) were administered. Overall scores and tiered accuracy were analysed across matched pairs and participant groups. Type and frequency of expressive naming errors were also coded.

Results: Relative to TD children, children with ABI had significantly lower overall expressive $(p=.001)$ and receptive $(p=.040)$ scores. Tier one accuracy was equivalent, while tier two expressive $(p=.001)$, but not receptive scores $(p=.056)$ were significantly poorer. Tier three receptive $(p=.034)$ and expressive $(p=.005)$ accuracy was also significantly lower in the ABI group. Naming errors were predominantly semantic in both groups though TD participants demonstrated a wider distribution of error types.

Conclusions: Preliminary findings support the use of tiered vocabulary assessment and naming error analysis to better understand vocabulary skills in school-age children with ABI. Outcomes also support the potential for targeting tier two and three vocabulary in this population within schools and communities. 
Session 4: Connecting through team practice in rehabilitation

\title{
Cognitive Rehabilitation Following Traumatic Brain Injury: A Survey of Current Practice in Australia
}

\author{
Marina Downing, ${ }^{1}$ Peter Bragge ${ }^{2}$ and Jennie Ponsford ${ }^{1}$ \\ ${ }^{7}$ Monash-Epworth Rehabilitation Research Centre, School of Psychological Sciences, Monash Uni- \\ versity, VIC, Australia \\ ${ }^{2}$ BehaviourWorks Australia, Monash Sustainable Development Institute, Monash University, VIC, \\ Australia
}

$\boldsymbol{R}$ ackground and objective: Cognitive impairments represent the greatest impediment to individuals with traumatic brain injury (TBI), therefore, cognitive rehabilitation is vital. Several sets of guidelines for cognitive rehabilitation have been published, including INCOG in 2014; however, little is known about current practice by therapists working with individuals with TBI. This study aimed to characterise current cognitive rehabilitation practices via an on-line survey of therapists engaged in rehabilitation in individuals with TBI.

Method: The 221 respondents were predominantly occupational therapists, neuropsychologists and speech pathologists with an average 9 years of clinical experience in cognitive rehabilitation and TBI. The survey documented demographic information, current practice of cognitive rehabilitation, resources used to inform cognitive rehabilitation and reflections on cognitive rehabilitation provided.

Results: Cognitive retraining and compensatory strategies were the most commonly identified approaches used in cognitive rehabilitation. Executive functioning was mostly targeted for retraining, whereas memory was targeted with compensatory strategies. Attentional problems were less frequently addressed. Client self-awareness, family involvement, team collaboration and goal-setting were seen as important ingredients for success.

Conclusion: Aspects of clinical practice are broadly consistent with guidelines. However, addressing the impediments to the delivery of cognitive rehabilitation is important to enhance quality of life for individuals with TBI.

\section{Implementing Inter-Disciplinary Goal Attainment Scaling in Clinical Practice}

Kerrin Watter, ${ }^{1,2}$ Mandy Nielsen, ${ }^{1,2}$ and Areti Kennedy ${ }^{1,2}$

${ }^{1}$ Acquired Brain Injury Transitional Rehabilitation Service, Division of Rehabilitation, Metro South Health, Brisbane, Australia

${ }^{2}$ The Hopkins Centre, Division of Rehabilitation Metro South Health and Griffith University, Brisbane, Australia

$\boldsymbol{R}$ ackground and objectives: In July 2016, a new rehabilitation service $\mathcal{B}$ model for adults with brain injury was implemented in Queensland. The acquired brain injury transitional rehabilitation service (ABI-TRS) commenced clinical service in January 2017, providing interdisciplinary team (IDT) community rehabilitation. Traditionally, Queensland's ABI rehabilitation services are provided using a multidisciplinary approach, with multi- and single-discipline goals. Establishing and maintaining new clinical practices, including IDT practice and IDT goal setting, can be challenging for teams. The ABI-TRS implemented IDT goal setting practices, including IDT goalattainment scaling (GAS) as standard care.

Method: A change management process was used to facilitate and maintain clinical change for IDT goal processes (including GAS-goals), via 
strategically planning service change, involving stakeholders, implementing change strategies and establishing an evaluation and review process. Specifically, this included identifying clinicians' skill, knowledge and experience; providing training and education; implementing supported goal setting. An expert review process involved experienced researchers providing feedback on IDT GAS goals. Team evaluation of processes also occurred; clinicians provided qualitative information regarding IDT goal setting; experience and confidence; barriers and facilitators. Supported goal setting and ongoing review processes have helped maintain new practices.

Results: IDT GAS goal-setting was successfully implemented within ABITRS, providing a consistent measure of goal attainment within an IDT service. The implementation and change management process, barriers and facilitators identified by the team and goal examples will be presented.

Conclusions: IDT GAS goals are feasible; and establishing and maintaining team change is enhanced by planned change management and support processes.

\section{Brain Injury Rehabilitation: Outcomes of Specialist and Non-specialist Units}

Tara Alexander, Jacquelin Capell, Frances Simmonds, and Diane Whiting

AROC, AHSRI, Innovation Campus, University of Wollongong, Australia

$\boldsymbol{R}$ ackground and aims: Brain injury rehabilitation enables those who have 3 experienced a brain injury to maximise their abilities and achieve optimal functioning. The Australasian Rehabilitation Outcomes Centre (AROC), established in 2002, is the rehabilitation medicine clinical registry for Australia and New Zealand. AROC collects standardised data against each inpatient rehabilitation episode of care, including traumatic and non-traumatic brain injury.

Method: This paper looks at the variation in rehabilitation outcomes following treatment in specialist units compared with non-specialist units for traumatic and non-traumatic brain injury. The influences on outcomes of factors such as time between injury and admission to rehabilitation, Functional Independence Measure (FIM) scores at admission and time spent in PTA are explored.

Results: Brain injury accounted for $3 \%$ of rehabilitation data submitted to AROC in 2017. Most non-traumatic brain injury patients were treated in nonspecialist units (79\%). Half of all traumatic brain injury patients were treated in specialist units (53\%). In comparison to non-specialist units, specialist units treat younger patients, have higher volumes of traumatic injuries and admit lower functioning patients. While the median LOS for all brain injury patients treated at specialist units has declined over the past five years, it is still five days longer than non-specialist units. Specialist units achieve greater functional improvement.

Conclusions: While there have been small changes in rehabilitation outcomes over the past five years most variation exists between whether the injury was traumatic or not and the type of unit the patient was treated in. 


\title{
Defining a Sustainable Workload for Staff Working in the New South Wales Brain Injury Rehabilitation Programs
}

\author{
Marion Fisher ${ }^{1}$ and Grahame Simpson ${ }^{2,3}$ \\ ${ }^{1}$ Brain Injury Rehabilitation Directorate, NSW Agency for Clinical Innovation, Sydney, Australia \\ ${ }^{2}$ Brain Injury Rehabilitation Research Group, Ingham Institute, Sydney, Australia \\ ${ }^{3}$ Brain Injury Rehabilitation Unit, Liverpool Hospital, Sydney, Australia
}

\begin{abstract}
$\boldsymbol{R}$ ackground and aims: Brain Injury Rehabilitation Programs (BIRP) in B NSW face the challenge of an ever-growing caseload because of the long term nature of the sequelae of brain injury. Furthermore, client re-engagement with the community rehabilitation teams tends to be episodic and unpredictable, and clinicians face a growing administrative burden associated with the introduction of funding authorities for people in serious accidents and with disabilities. This project aimed to define a sustainable workload for BIRP staff. Method: A model of workload capacity was developed following a comprehensive literature review, focus groups and semi-structured interviews with BIRP staff. Data that incorporated all aspects of the model were collected and analysed from four BIRP services. Clinicians completed the Case Manager Personal Efficacy Scale as an indicator of manageable caseload. Data were also collected on possible predictors of workload capacity including clinician features, caseload, work-tasks and workplace/ governance features.

Results: The strongest predictor of time required for caseload management was an intensity rating scale (need for service supports). Client complexity was not a strong predictor, neither were clinician characteristics. An abbreviated tool (the Workload Capacity Tool, WCT) for tracking sustainable workloads was created from the predictive data elements including non-clinical work, uncompleted work tasks, supervision structure and workplace specific features.

Conclusions: This project showed that a sustainable workload could be defined by a limited number of features. The WCT provides a reflective resource for considering self-efficacy and stress in relation to caseload, other work-tasks, supervision and tasks that were not completed.
\end{abstract}

\section{Exploring eHealth 'tribes and tribulations' in Interdisciplinary Rehabilitation for People with a Traumatic Brain Injury (TBI)}

\author{
Monique Hines, ${ }^{1}$ Melissa Brunner, ${ }^{1,2}$ Simon Poon, ${ }^{3}$ Mary Lam, ${ }^{4}$ Vivienne Tran, ${ }^{1}$ \\ Dan $\mathrm{Yu}^{3}{ }^{3}$ Leanne Togher, ${ }^{1,5}$ Tim Shaw, $^{1}$ and Emma Power ${ }^{1}$ \\ ${ }^{7}$ Faculty of Health Sciences, The University of Sydney, Lidcombe, Australia \\ ${ }^{2}$ Faculty of Education and Arts, The University of Newcastle, Callaghan, Australia \\ ${ }^{3}$ Faculty of Engineering and Information Technologies, The University of Sydney, Sydney, Australia \\ ${ }^{4}$ Faculty of Health, University of Technology Sydney, Ultimo, Australia \\ ${ }^{5}$ Moving Ahead, NHMRC Centre of Research Excellence in Brain Recovery, School of Psychology, \\ The University of New South Wales, Sydney, Australia
}

$\boldsymbol{R}$ ackground and aims: The potential of eHealth for supporting interdisci3 plinary care is vast, with benefits in maximising efficiency and improved quality of care. As yet, it is unclear whether this potential is being realised in traumatic brain injury (TBI) rehabilitation practice. This study aimed to explore health professionals' experiences of eHealth and their attitudes towards using technology to support interdisciplinary rehabilitation for people after TBI.

Method: This qualitative study used narrative analysis of data collected from three focus groups and one individual interview with health professionals 
( $n=17)$ working in TBI rehabilitation across regional and metropolitan New South Wales, Australia.

Results: Participants were largely enthusiastic about eHealth and its potential to support interdisciplinary practice in TBI rehabilitation. However, they encountered various issues that disconnected them from their work. These issues related to the (a) design and access to electronic medical records, (b) technology, (c) eHealth implementation and (d) eHealth processes. Health professionals attempted to make the most of the available eHealth systems and processes, but often felt they were unsuccessful in providing efficient, quality, client-centred services.

Conclusions: There is an urgent need to address sources of disconnection experienced by health professionals. Specifically, attention to the design and access to eHealth technologies and the development of supportive policies and procedures that underpin eHealth and interdisciplinary practice, is needed to achieve effective connections and collaborations in rehabilitation. By addressing these issues, service organisations may be better placed to support health professionals to deliver optimal client care.

\title{
Combining Research with Practice: Reflections on an Embedded Evaluation of a New Acquired Brain Injury Service
}

\author{
Mandy Nielsen, ${ }^{1,2,3}$ Areti Kennedy, ${ }^{1,2}$ and Kerrin Watter ${ }^{1,2}$ \\ ${ }^{7}$ Acquired Brain Injury Transitional Rehabilitation Service, Division of Rehabilitation, Metro South \\ Health, Brisbane, Australia \\ ${ }^{2}$ The Hopkins Centre, Division of Rehabilitation Metro South Health and Griffith University, Brisbane, \\ Australia \\ ${ }^{3}$ School of Human Services and Social Work, Griffith University, Brisbane, Australia
}

$\boldsymbol{B}$ ackground and aims: While post-discharge support is identified as crucial during the transition from hospital to home post-Acquired Brain Injury (ABI), research highlights unmet support needs of people with $\mathrm{ABI}$ and their families during this period. The Acquired Brain Injury Transitional Rehabilitation Service (ABI TRS) is a 5-year pilot project within the Division of Rehabilitation, Princess Alexandra Hospital (PAH), Brisbane. The service aims to facilitate earlier discharge from hospital and improve community outcomes for people with $\mathrm{ABI}$ and their families. Integral to the pilot project is an embedded evaluation to critically appraise process, impact and economic outcomes. This involves balancing outcome evaluation of clinical interventions with the processes of integrating the model within an existing continuum of brain-injury services.

Method: Implementing a new clinical model may require whole of system change and is a complex, multi-level process. A mixed method research design incorporating five staged components is in progress, with completion mid2021. Integral to this is reflexive monitoring of the evaluation design and responsiveness to ongoing service implementation.

Results: The process of dynamically evaluating a new service from planning to implementation will be discussed. Learnings from the first year of combining implementation with evaluation will be presented.

Conclusions: The evaluation of the ABI TRS pilot project is providing insights into the process of implementation and integration of research within an emergent and dynamic clinical practice environment. These insights may assist future projects of this kind. 


\title{
Neurocognitive Functioning in Behcet's Disease
}

\author{
Caroline Fisher, and Coco Bernard \\ Allied Health - Psychology, Royal Melbourne Hospital, Melbourne Health, Melbourne, Australia
}

$\boldsymbol{R}$ ackground and objectives: Behcet's disease (BD) is a vascular and mulB tisystem inflammatory disorder, characterised by oral and genital ulcerations, skin and eye lesions and vascular abnormalities. Central nervous system involvement occurs in a proportion of sufferers, in the form of brainstem or multifocal lesions, myelopathy, cerebral abnormalities and optic neuropathy, cerebral venous thrombosis, intracranial hypertension syndrome and acute meningeal syndrome. This neurological involvement is often referred to as Neuro-Behcet's disease (NBD). The objective of this research is to determine the neurocognitive profile of $\mathrm{BD}$.

Method: A systematic review into the neurocognitive features of Behcet's disease. This review was designed using PRISMA guidelines, and a quality assessment system adapted from the Cochrane handbook was utilised. The search included all published, group comparison, group categorisation, case series and case study research up to April 2017.

Results and conclusions: The search identified 312 papers after duplicates were removed, with 30 papers meeting the criteria for inclusion, this included 13 group comparison studies and 17 case series papers.

The results of the neuropsychological assessment data by neurocognitive domain, will be presented along with the methodological limitations of the current evidence base. The implications of neurocognitive impairment for Behcet's suffers will be outlined, and the current debate regarding 'silent' NBD discussed.

\section{Session 5: How to Session 1}

\section{What Ingredients Contribute to Effective Treatment of Social Cognition?}

\author{
Barbra Zupan ${ }^{1}$ and Leanne Togher ${ }^{2}$ \\ ${ }^{1}$ Central Queensland University, Queensland, Australia \\ ${ }^{2}$ University of Sydney, Sydney, NSW, Australia
}

Cynopsis: Evidence-based treatments for social cognition are on the rise. $\mathcal{W}$ While some of these treatments address a specific component of social cognition (e.g., facial emotion recognition), others are more general, targeting a number of cognitive-communication deficits within one programme. Regardless, these programmes share a range of techniques and strategies that appear to contribute to the individual's overall success in treatment. For example, the majority of these evidence-based social cognition treatments incorporate extensive and mass practice, positive reinforcement, roleplay and generalisation. In response to the Rehabilitation Treatment Taxonomy (RTT; Dijkers, 2014), a conceptual framework aimed to classify what it is we do as clinicians or have our patients do during rehabilitation, we will identify these ingredients and discuss how they are likely to make a difference for the patient. Being able to identify that techniques and strategies are contributing to improved outcomes for our patients is essential for building the evidence required by third party payers who may refuse to pay for treatments that are not clearly defined or evidence-based. This seminar is the first step in assisting clinicians to identify the ingredients, defined by the RTT as observable and measurable actions, they use in therapy to target social cognition. Factors that may additionally impact 
the implementation and measurement of these techniques (e.g., person factors and environmental factors) and strategies will also be discussed.

Level Aimed For: Basic and Intermediate

Learning Objectives: (1) To identify the common ingredients used in evidence-based treatments for social cognition and discuss ways in which these can be implemented and measured in current practice; (2) To identify factors that facilitate or hinder the use of these techniques in current practice

\title{
Session 6: How to Session 2
}

\section{How to Develop the Capacity of National Disability Insurance Scheme Participants with Acquired Brain Injury and the Workforce they Engage with: Investigations, Tips, and Tools}

\author{
Libby Callaway, ${ }^{1,2}$ Sue Sloan, ${ }^{3}$ Jan Mackey, ${ }^{4}$ Prue Morgan ${ }^{5}$ and Emma Gee ${ }^{6}$ \\ ${ }^{1}$ Occupational Therapy Department, School of Primary and Allied Health Care, Monash University, \\ Frankston, Australia \\ ${ }^{2}$ Neuroskills Pty Ltd, Sandringham, Australia \\ ${ }^{3}$ Osborn Sloan and Associates, Kew, Australia \\ ${ }^{4}$ Applied Communication Skills, Clifton Hill, Australia \\ ${ }^{5}$ Physiotherapy Department, School of Primary and Allied Health Care, Monash University, Frankston, \\ Australia \\ ${ }^{6}$ Emma Gee Inc., Cotham, Australia
}

\section{Session Synopsis}

7 he National Disability Insurance Scheme (NDIS) radically changes the 1 framework for delivery of support to people with acquired brain injury (ABI). With initial Scheme roll-out, significant risks have emerged that may challenge a participant's ability to access community rehabilitation. For instance, the funding allocated to capacity building (rehabilitation) during NDIS planning has initially been extremely low. If this funding is available, there is often insufficient workforce to deliver the necessary skilled supports. This 'How To' session will outline an NDIS Innovative Workforce Fund project delivered with people with ABI living in Specialist Disability Accommodation (SDA), and their support workforce. Key project findings and outputs will be presented, including:

1) An activity audit with people with ABI living in SDA, using the NDIS participant outcome domains;

2) A suite of digital workforce training, made available to the ABI sector;

3) A mixed-methods face-to-face and on-line health professional student training model in SDA;

4) A manualised approach to engaging people with $\mathrm{ABI}$, and training their supporters, in the activities of the NDIS-defined concept of an 'ordinary life'.

Level aimed for: Basic to Intermediate

Learning objectives:

At the end of this session, participants will

1. Develop knowledge of evidence-based community ABI rehabilitation practice in SDA, within an NDIS policy context; 
2. Understand opportunities to maximise therapy supports linked to outcomes in the NDIS planning process;

3. Have access to a range of tools and understanding of the NDIS frameworks, workforce training strategies, and support processes to achieve capacity building with people with $\mathrm{ABI}$.

\title{
Session 7: How to Session 3
}

\section{Utility of Brain Neuroimaging in Dementia: The good, the Bad, and the UglyUtility of Brain Neuroimaging in Dementia: The good, the Bad, and the Ugly}

Olivier Piguet $^{1,2}$

${ }^{1}$ The University of Sydney, School of Psychology and Brain \& Mind Centre, Sydney, Australia

${ }^{2}$ ARC Centre of Excellence in Cognition and its Disorders, Sydney, Australia

Cynopsis of session: Dementias are progressive neurodegenerative brain $\int$ conditions that manifest clinically in many different forms, depending on the type, location and severity of the underlying pathology. Early and appropriate diagnosis of dementia is becoming a pressing issue, given the ageing of the population and predicted increase in the number of dementia cases in the coming decades. Neuro-imaging has become a central tool to help with the identification and differential diagnosis of dementia syndromes, as reflected by their inclusion in the current clinical diagnostic criteria. These investigations, however, are expensive and may not always offer an appropriate answer. This session will provide an overview of the structural and functional neuro-imaging profiles of the main dementia syndromes (Alzheimer's disease, frontotemporal dementia, dementia with Lewy bodies). In particular, the session will focus on structural MRI (volumetrics, diffusion tensor imaging) and positron emission tomography (glucose, pathology-specific ligands), and will discuss the benefits and limitations of these techniques in clinical settings. Tips and suggestions on how to best integrate neuro-imaging data in clinical practice will be presented.

Level aimed for: Basic to Intermediate

Learning objectives: By the end of the session, attendees will be able to: (i) understand the main types of brain neuro-imaging techniques and their purposes, (ii) recognise the neuro-imaging profiles of the main dementia syndromes on structural and functional imaging and (iii) relate neuro-imaging findings to other clinical data (e.g., cognitive, functional).

Plenary 2

\section{Building Connection: Interventions to Improve Vocational, Family and Psychological Outcomes After Severe TBI}

\author{
Grahame Simpson \\ Brain Injury Rehabilitation Research Group, Ingham Institute of Applied Medical Research
}

\footnotetext{
$A$ dults sustaining moderate to severe traumatic brain injury (TBI) often experience social exclusion, isolation and alienation from key domains of social life. These outcomes are the result of systemic, social and psychological factors. The impact of such experiences can result in a range of negative physical and psychological morbidity for people with TBI. Seeking to rebuild connection in the face of these challenges involves the development of
} 
appropriate models and interventions that foster participation and reengagement post-injury, across vocational, family and psychological domains. Recent developments at a national level have resulted in additional barriers for people with TBI seeking to access appropriate vocational rehabilitation. The results of a trial of a dual track (return to pre-injury employment, access to new employment) vocational rehabilitation intervention that aimed to increase vocational participation after severe TBI will be described. Connection to family is also key to social integration. However, most research to date has interviewed single family members only, and so little is known about how care for people with TBI is distributed across family networks. Social network analysis provides a method to identify these patterns of care and can help in understanding the linkages between people with TBI and their family members, and may also have implications for understanding the longer-term sustainability of family support. Finally, at a psychological level, the result of recent trials of psychological interventions for civilians and military veterans with moderate to severe TBI will be reported. These interventions have aimed to reduce psychological morbidity and enable a re-connection with hope and adaptive responses to the challenges of living with severe TBI.

\section{ABSTRACTS - FRIDAY POSTERS}

\section{Using a Participatory Action Research Approach to Facilitate the Implementation of the Instrumental Activities of Daily Living Profile in Clinical Practice}

Carolina Bottari, ${ }^{1,2}$ Dahlia Kairy, ${ }^{1,2}$ Priscilla Lam-Wai Shun, ${ }^{1,2}$ Catherine Ouellet, ${ }^{1}$ Christine Magnan, ${ }^{1}$ Lise Poissant, ${ }^{1,2}$ Deirdre Dawson, ${ }^{3}$ and Bonnie Swaine ${ }^{1,2}$

${ }^{1}$ Centre for Interdisciplinary Rehabilitation Research of Greater Montreal, Canada

${ }^{2}$ Faculty of Medicine, School of Rehabilitation, Université de Montreal

${ }^{3}$ Department of Occupational Science and Occupational Therapy \& Graduate Department of Rehabilitation Sciences, University of Toronto

T ntroduction: The Instrumental Activities of Daily Living (IADL) Profile was developed to measure independence in complex situations in home and community environments through the lens of executive function deficits, and to guide treatment interventions. However, a gap remains between the evidence and actual tool use, which supports the importance of promoting its implementation using effective knowledge translation activities (KTA).

Objectives: (1) To identify perceived barriers to the clinical use of the IADL Profile at the individual and organisational level; (2) to develop and implement KTA to facilitate its use.

Methods: Participatory action research including six rehabilitation centres in Canada. 61 occupational therapists working in TBI programmes completed a 64-item questionnaire developed to identify the perceived barriers and facilitators to the use of the IADL Profile after an initial training (T1, $n=61$ ) and then again after a 6-month trial period (T2, $n=29)$. A multi-faceted knowledge translation strategy called the IADL Profile Knowledge Implementation Toolkit (I-KIT) was developed to address obstacles to the uptake of the tool. Throughout the study, clinicians tested, commented, questioned and improved the I-KIT. The I-KIT consists of: web-based learning, an interactive educational workshop, website, on-line support, outreach visits and clinical champions. 
Results: Subsequent to the trial period, participants reported feeling more confident about administering the IADL Profile. I-KIT activities were globally considered as relevant, with satisfaction ranging between 58 and 100\%.

Conclusions: I-KIT activities were positively perceived to facilitate clinical use of the IADL Profile. The trial period component of the I-KIT increased perceived clinician self-efficacy in administering the IADL Profile.

\section{Consumer Focused Inpatient Peer Support Group: Final Results}

Simone Cahill, Annerley Bates, and Wilma Schouten

Brain Injury Rehabilitation Service, Princess Alexandra Hospital, Brisbane, Australia

$\boldsymbol{R}$ ackground: Psychosocial stressors can influence an individual's ability 3 to focus and engage in a therapy programme after a brain injury (Anson \& Ponsford, 2006; Hibbard et al 2002; Albert et al, 2002). Peer support groups provide a valuable avenue for patients to increase their knowledge of brain injuries, improve their ability to cope with psychosocial stressors, and thus enhance their quality of life. The majority of group interventions are held one or more years post injury when patients have been discharged from hospital. Objectives: This study had three primary objectives: (1) address the psychosocial needs of patients, (2) provide a forum to share experiences and (3) psycho education as a way of increasing patient insight into the recovery process after a brain injury.

Methods: A five year longitudinal evaluation of an inpatient brain injury rehabilitation peer support programme was completed in September 2017. The survey used a five-point Likert scale to investigate the emotional distress of patients with an ABI and their level of comfort in sharing their experiences with peers and staff.

Results: The results from the survey indicate that attendance at the group allowed patients to share experiences, discuss problems, explore solutions and improve relationships with peers and staff on the ward.

Conclusions: This programme is innovative, patient centred and designed around empirical research to meet the needs of patients with a brain injury. As a result, the group is now an integral part of the inpatient rehabilitation programme that is valued by the unit.

\section{The Communication Gap Between Staff and People with Aphasia: There's an App for that!}

Marcella Carragher, ${ }^{1}$ Robyn O’Halloran, ${ }^{1}$ Hilary Johnson, ${ }^{1}$ Nicholas Taylor, ${ }^{2}$ Torab Torabi, ${ }^{3}$ and Miranda Rose ${ }^{1}$

${ }^{1}$ Community and Clinical Allied Health, La Trobe University, Melbourne, Australia

${ }^{2}$ Department of Rehabilitation, Nutrition and Sport, La Trobe University, Melbourne, Australia

${ }^{3}$ Department of Computer Science and Information Technology, La Trobe University, Melbourne, Australia

$\boldsymbol{R}$ ackground and objectives: Effective communication between health professionals and patients is essential for safe, efficacious and patientcentred care. Transition between services and episodes of care represent weak spots in the continuum of care for quality and safety. Particularly, at risk are those patients who find themselves suddenly living with aphasia, a communication disability caused when the stroke damages the language networks of the brain. Aphasia presents serious challenges to effective healthcare communication and health professionals feel under-skilled and under-resourced to communicate with this patient population. This study considers the usefulness 
of mobile technology to address the communication gap between patients and health professionals.

Method: A participatory co-design was used to engage consumers (people with aphasia, family members, acute and subacute health professionals) in the design of an app. Consumers attended a series of focus groups and workshops. Initial focus groups were exploratory in nature, while later workshops focused on getting hands-on experience with a pilot app and providing feedback to refine content, design and functionality.

Results: Following extensive consumer engagement and refinement, the Aphasia App has been produced to support healthcare communication between staff and patients with aphasia. The content and design of the app will be presented, along with results from a current pilot study of the app's utility in healthcare settings.

Conclusions: The Aphasia App is a world-first in supporting healthcare communication during the hospital admission after stroke. Future studies will investigate the efficacy of the app across clinical settings.

\title{
Learning to SIFT IT: A Proof of Concept Study for a Social Cognition Skills Group After Traumatic Brain Injury.
}

\author{
Anneli Cassel, ${ }^{1,2}$ Skye McDonald, ${ }^{1,2}$ and Michelle Kelly ${ }^{2,3}$ \\ ${ }^{1}$ School of Psychology, University of New South Wales, Sydney, Australia \\ ${ }^{2}$ Moving Ahead Centre of Research Excellence in Brain Recovery, University of New South Wales, \\ Sydney, Australia \\ ${ }^{3}$ School of Psychology, University of Newcastle, Newcastle, Australia
}

R ackground and aims: The impact of social cognitive deficits after traumatic brain injury (TBI) are increasingly recognised. Despite this, few studies have examined the feasibility and efficacy of remediation efforts in this area. In this study, we aimed to demonstrate proof of concept for a comprehensive group social cognition treatment programme in this population. Here, two cases are presented.

Method: A 14-session social cognition intervention was piloted with two males ('Greg', aged 30, and 'Aaron', 54) who had both experienced a TBI three and five years ago, respectively. Both reported difficulties in social situations: Greg had difficulties maintaining stable employment; Aaron had a strained relationship with his wife. A detailed social cognitive baseline assessment was conducted before treatment: Greg had mild deficits spanning emotion perception and cognitive perspective taking; Aaron had more extensive deficits, alexithymia and low empathy. Measures were repeated post-treatment and at three-month follow-up.

Results: Both participants attended regularly but responded to the group differently: Greg achieved his goals, whilst they remained unchanged for Aaron. Greg demonstrated reliable and clinical improvement post-treatment on social cognitive testing, social anxiety and Quality of Life. Aaron showed some reliable improvement on social cognitive measures but largely remained similar to baseline.

Conclusions: This is the first group treatment programme that comprehensively targeted interacting social cognitive processes after TBI. Preliminary findings suggest the group warrants further investigation: both participants completed the programme with good attendance and showed some evidence of both reliable and clinical improvements. 


\title{
Developing a Standardized Approach to Assessment of Young Individuals with Disorders of Consciousness
}

Tanya Cavlovic and Georgina Hett

Disability Services, Brightwater Care Group, Perth, Australia

$\boldsymbol{R}$ ackground and aims: Accurate diagnosis for individuals with disorders of consciousness (DOC) can be challenging. While standardised behavioural assessments are considered 'gold standard' for detecting signs of consciousness, there are no simple tests for awareness, and clinician knowledge and experience working with this population is often varied and limited. The aim of this study was to develop a standardised approach to assessment of DOC.

Method: (1) Conducted a literature review to inform the current evidence on assessment of DOC; (2) Selected and trialled The Disorder of Consciousness Scale (DOCS) over five months with five individuals aged 21-48 years with a DOC following an acquired brain injury, whilst residing at our transitional and residential care facility; and (3) Surveyed local health service providers to determine what assessment tools /protocols were being utilised.

Results: The DOCS proved easy to use, and supported assessment and care planning over and above existing practises. DOCS was one of the few structured assessments recommended internationally, however, the Coma Recovery Scale-Revised (CRS-R) was identified as the tool of choice. The survey highlighted that assessment of this population remains limited and inconsistent locally, whilst the most widely used (or proposed for use) tool, was the CRSR.

Conclusions: This process has shaped the development of a standardised assessment protocol for DOC relevant to a post-acute setting. Having a common frame of reference to facilitate collaboration with local services, in the interest of accurate assessment and care planning for individuals with DOC, was an influential factor in assessment choice.

\section{Simulator Assessment for Obtaining Medical Clearance for Driving Following Severe Traumatic Brain Injury: A Case Study Example}

\author{
David Coleopy ${ }^{1}$ and Nicole de la Perrelle ${ }^{2}$ \\ ${ }^{1}$ Paraquad, Dulwich, South Australia, Australia \\ ${ }^{2}$ Faculty of Medicine, Nursing and Health Sciences, Flinders University, Adelaide, South Australia, \\ Australia
}

$\boldsymbol{B}$ ackground and aims: Individuals with traumatic brain injury (TBI) may require Occupational Therapy (OT) Driver Assessment to determine capacity to return to driving following their injury, however, obtaining medical clearance to complete an On Road assessment can be challenging and requires collaborative and multi-layered assessment and treatment processes. In this case study, a 34 year old man who sustained a severe TBI in 2007 underwent a comprehensive OT Driver assessment and rehabilitation process, including the use of driving simulators to determine his fitness to undertake an on road assessment.

Method: OT Driver Assessment including extensive clinical assessment of physical, visual and cognitive capacity. Subsequent assessment included a go kart driving assessment, use of two driving simulators, and an on road assessment with OT and driving instructor at a private club driving track.

Results: Clinical assessments indicated a variety of mild cognitive deficits related to driving although results were conflicting. The use of the two 
driving simulator sessions using a virtual reality headset and one session on an immersive and adapted clinical simulator more closely demonstrated driving capacity, challenges, issues and behaviours demonstrated when driving a vehicle on a driving track with an instructor. Subsequently, the individual has been determined as having functional capacity to progress to on road driving assessment, although medical fitness remains complicated due to medication changes.

Conclusions: Use of multi-layered OT Driver Assessment processes including use of driving simulators can provide clinically significant information compared to clinical off road assessment alone.

\title{
An Examination of Individuals with Assault-related Traumatic Brain Injuries (TBIs), their Informal Caregivers, and their Contact with the Criminal Justice System
}

\author{
Durc Fahrenhorst-Jones, ${ }^{1}$ Annerley Bates, ${ }^{2}$ and Lyndel Bates ${ }^{1}$ \\ ${ }^{1}$ School of Criminology and Criminal Justice and Griffith Criminology Institute, Griffith University, \\ Brisbane, Australia \\ ${ }^{2}$ Brain Injury Rehabilitation Service, Princess Alexandra Hospital, Brisbane, Australia
}

\begin{abstract}
$B$ ackground and aims: There is limited information surrounding individuals with assault-related traumaticbrain injuries (TBIs) and their informal caregivers (Bates et al., 2016). This is problematic as violence is a public health concern (Krug, Dahlberg, Mercy, Zwi, \& Lozano, 2002) and assault-related TBIs comprise of a distinct clinical sub-group (Kim et al., 2013). Limited research suggests individuals with TBIs may receive more support from the criminal justice system than the health care system (Degeneffe \& Bursnall, 2014). Families are generally informal caregivers of individuals with TBIs (Foster et al., 2012). Subsequently, there is a need to examine individuals with assault-related TBIs, their informal caregivers, and their contact with the health care and criminal justice systems.

Method: Retrospective administrative data from Queensland Health was analysed. From 2011 to 2015, 84 individuals with assault-related TBIs were referred to Social Workers for review at a State-wide brain rehabilitation service (BIRS).

Results: $18 \%$ of the total TBI sample $(n=467)$ received their injury as the result of an assault. Injured persons were generally male $(86.2 \%)$ and the victim (as opposed to the perpetrator of assault; 80.0\%). Many assaults also involved alcohol $(29.2 \%)$. Females were the most common informal caregivers (66.2\%). Injured persons generally had contact with Police services $(46.2 \%)$ and victims support providers $(26.2 \%)$. Informal caregivers had no recorded contact with the criminal justice system.

Conclusions: Findings suggest future interventions targeting this population should include male victims, partners, parents and families generally. Further research is required to examine informal caregivers contact with the criminal justice system.
\end{abstract}

\section{Driving Remediation Following Acquired Brain Injury: A Case Study Series}

Janelle Griffin, ${ }^{1}$ Louise Bassingthwaighte, ${ }^{1}$ and Jenny Fleming ${ }^{2}$

${ }^{1}$ Department of Occupational Therapy, Princess Alexandra Hospital, Brisbane, Australia

${ }^{2}$ School of Health and Rehabilitation Sciences, The University of Queensland, Brisbane, Australia

$\mathcal{R}$ ackground and aims: Occupational therapy driving assessments aim to evaluate the impact of medical conditions on a person's capacity to drive. Whilst the benefits of acquired brain injury (ABI) rehabilitation are 
well documented, there is very little evidence concerning the efficacy of onroad driving remediation programmes. Furthermore, the capacity of a person to sustain any improvements gained through driving rehabilitation over time have not been researched. This study aimed to describe the outcomes of participants in a driving remediation trial project.

Method: Participants were eight people with acquired brain injury in a pilot wait-listed randomised control trial. Both the control and intervention group completed a baseline assessment battery including a standard off-road and onroad assessment and psychosocial measures. The intervention group completed an on-road driving rehabilitation programme over six to eight weeks, whilst the control group received no intervention. For both groups, the assessment battery including on-road driving performance was then repeated by a blind assessor. The control group then completed the driving intervention and were assessed. Finally, both groups completed a reassessment six months post intervention.

Results: There was general support for the effectiveness of individualised occupational therapy on-road driving remediation. Maintenance of driving remediation gains after six months in terms of driving performance and psychosocial factors was inconsistent. Increased self-efficacy was evident across all participants following completion of the programme.

Conclusions: Findings will provide consumers and health professionals with greater knowledge about the return to driving process including the efficacy of on-road driving remediation and psychosocial factors to consider.

\section{Enabling Social Drinking in a Supported Community Setting Following Acquired Brain Injury}

Nicky Brosnan, and Rachel King

Oats Street Rehabilitation Programme, Brightwater Care Group, Perth, Australia

$B$ ackground and objectives: Alcohol plays a major role in society. Following an acquired brain injury (ABI), people often do not know whether they can or should consume alcohol and have limited access and opportunity to do so. Some doctors may not provide clearance for alcohol consumption after $\mathrm{ABI}$ and for some this is not acceptable. Without collaboration and negotiation this can cause frustration and disappointment. The aim of this programme was to increase understanding for both clients and staff about alcohol and brain injury, and provide people with ABI an opportunity to consume alcohol in a supported environment.

Method: Two groups of $n=4$ clients (total $n=8$ ) with an ABI completing post-acute rehabilitation, completed education on ABI and alcohol, signed a contract of attendance for the programme and were supported by one Disability Support Worker to attend a local venue within the community for 6-week blocks. Focus group, questionnaire and interview data were collected pre and post supported outings.

Results: Staff and participant attitudes towards drinking improved. Individuals became more sociable on site, community inclusion increased and mood improved. Improvements were observed on measures of mental health and wellbeing, inclusion and understanding of ABI and alcohol. Individuals looked forward to the group and the opportunities it brought. Observations of group interactions highlighted the importance of community integration.

Conclusions: Supporting people in ABI rehabilitation to consume alcohol at appropriate levels within a social community environment led to decreased incidences of excessive alcohol consumption as well as decreased social isolation. 


\title{
Social Work and the Psychosocial: Understanding the Practice Challenges at the Policy/Practice Interface in Community Neuro-Rehabilitation
}

\author{
Brooke Kooymans ${ }^{1,2}$ \\ ${ }^{1}$ School of Human Services and Social Work, Griffith University, Brisbane, Australia \\ ${ }^{2}$ Rehability Australia, Brisbane, Australia.
}

\begin{abstract}
$\boldsymbol{B}$ ackground and objectives: Psychosocial needs of individuals with acquired brain injury (ABI) are often multi-faceted indicating the involvement of multiple professional disciplines and services. Yet, typically policy frameworks and organisational imperatives dictate the provision and delivery of services, which creates challenges and tensions for front-line workers. This paper aims to critically examine how the policy and organisational contexts of community neuro-rehabilitation influence social work practice and their approach to meeting the psychosocial needs of individuals with ABI.

Method: A critical appraisal of the literature was undertaken to address the aim. Relevant electronic databases, including CINAHL, Medline, Cochrane, Pubmed, psycBITE, PsycINFO and Scopus were searched. Relevant papers meeting the criteria were identified with content assessed and reviewed, for inclusion.
\end{abstract}

Results: The results demonstrate that policy and legislation directly impact on the delivery of services. Policy drivers shape service priorities with tangible needs, such as function, more of a focus than needs such as psychosocial. This context therefore influences and shapes front line workers responses and service delivery, where workers are using discretion and autonomy to negotiate and interpret the policy/legislation to deliver services at the street level.

Conclusions: There is limited knowledge developed to date that specifically addresses social work and the psychosocial, within the policy climate. Further research is warranted to gain more clarity, understanding and knowledge of how social workers negotiate the policy/practice interface, and how this defines their clinical work. Such knowledge will potentially enable improved social work practices and improved outcomes for individuals with ABI to be achieved.

\section{Are Therapists' Factors Associated with Client-Centred Goal Setting in Brain Injury Rehabilitation?}

Caitlin Page, Sarah Prescott, Emmah Doig, and Jennifer Fleming

School of Health and Rehabilitation Sciences, The University of Queensland, Brisbane, Australia

$\boldsymbol{B}$ ackground and aims: Challenges may arise when working with clients with acquired brain injury (ABI), due to cognitive impairments. A strong partnership between therapist and client underpins client-centred practice and may facilitate collaborative goal setting despite these changes. This study explores the relationships between therapists' skills, confidence, professional background and experience and the level of therapeutic alliance and clientcenteredness of goal setting sessions with clients with ABI.

Method: This cross-sectional study involved 19 multidisciplinary therapists working in ABI rehabilitation with 44 community-dwelling clients. Clientcenteredness and therapeutic alliance were measured from the client's perspective using the Client-Centeredness of Goal Setting Scale, Canadian Occupational Performance Measure and revised Helping Alliance Questionnaire. Therapists' data were collected using a customised survey.

Results: Survey data revealed that therapists perceived clients with ABI to have less understanding about what they need to work on compared to clients without ABI. There was no difference in client-centeredness between occupational 
therapists and other health professionals. Correlations between therapists' confidence, experience or skill and client-centeredness or therapeutic alliance were weak and non-significant. Significant relationships were found between therapists' years of experience and their self-perceived skill and confidence level in goal setting.

Conclusion: This study showed a lack of association between therapists' factors and the level of client-centeredness or therapeutic alliance in goal setting. Training activities that increase therapists' confidence, skills and encourage reflection on their intrinsic beliefs about clients' abilities, may be beneficial to enhance client-centred goal setting particularly for recently trained therapists or therapists new to the ABI rehabilitation field.

\title{
ABSTRACTS - SATURDAY 5TH MAY
}

\section{Plenary 3}

\section{Lifeworld-led Rehabilitation - A New Approach to Support Psychosocial Wellbeing Following Brain Injury}

Caroline Ellis-Hill

Bournemouth University, Bournemouth, UK

\begin{abstract}
$A$ traditional approach to $\mathrm{ABI}$ rehabilitation contributes many useful developments in physical and psychological rehabilitation and recovery. As clinicians we have expertise, we carry out observations and work out what needs doing to improve things for people living with ABI in the future. However, there is a danger that only looking in this objective scientific way can create unintended cultural and practice limitations. These include: (i) separation between clinician and (a) service users, (b) their own feelings and (c) their own moral compass (ii) clinicians focusing on what can be measured/assessed rather than what they feel is important and (iii) a need for control and the associated blame culture. Overall this can lead to a reduction in wellbeing for all concerned.

A lifeworld-led approach to rehabilitation has a very different underlying logic and offers us the ability to prioritise subjective experience, which in turn creates new understandings and connections. The approach is based on the philosophical work of Husserl. Our lifeworld is our everyday flow of life as we experience it, or what our life feels like from the inside out. When accessing lifeworld knowledge (a) we are in the present, in our bodies, (b) open to "not knowing' and curious, (c) in a safe space (d) where learning is shared. This allows connection, creativity and confidence to flourish. Three early studies based on these principles, (a) the HeART of stroke project, (b) Humanising care on a stroke ward and (c) the role of the expert companion in long-term ABI services, have shown to have powerful effects and will be discussed in this presentation.
\end{abstract}

\section{How Do We Do Person-Centeredness? Building Connectivity, Trust and Capability in the Midst of an Unstable Reality}

\author{
Nicola Kayes \\ University of Auckland, Auckland, New Zealand
}

$\boldsymbol{P}$ erson-centred practice has received widespread endorsement across health settings and contexts, and is understood to be an important and 
positive movement in rehabilitation. Despite this, there remains a lack of conceptual clarity over what person-centeredness is and how to operationalise it for practice, potentially contributing to the biomedical model remaining the prevailing discourse. An emphasis on increased patient choice, patient involvement in decision making, and increasing patient capacity for self-management, has become a proxy for person-centred practice, in lieu of a more fundamental shift in practice. While existing models offer us some insight into what person-centred practice is, there is little consideration of how it might embedded in practice; hampering meaningful progress. Given this, we conducted a secondary analysis of qualitative data exploring patient and practitioner perspectives of the therapeutic encounter in neurorehabilitation to answer the question: How do the voices of our participants inform us about how to do person-centeredness? In this presentation, I will present our findings, and discuss their implications for practice and the field.

\title{
Session 8: Understanding and Meeting the Needs of Everyday Living
}

\section{Connecting and Collaborating in Return to Work: Learning from the Perspective of Those with Acquired Brain Injury and Service Providers}

\author{
Adam Grope, ${ }^{1}$ Alice Dwyer, ${ }^{1}$ Damian Canavan, ${ }^{1}$ Melissa O'Brien, ${ }^{2}$ Brincat Freddie ${ }^{3}$ \\ Ada Caruana, ${ }^{3}$ Lauretta Giannone, ${ }^{4}$ Jan Rolan, ${ }^{4}$ and Liz Williams, ${ }^{4}$ \\ ${ }^{1}$ Private citizens; Multiple Solutions, Australia \\ ${ }^{2}$ WorkPlace Solutions, Australia \\ ${ }^{3}$ Community Bridging Services, Australia \\ ${ }^{4}$ Brain Injury Rehabilitation Community and Home (BIRCH) South Australian Brain Injury Rehabili- \\ tation Services, Australia
}

$\boldsymbol{B}$ ackground and aims: Return to work (RTW) is an important part of re-

3 habilitation and recovery for many individuals after their illness or injury. Published literature and anecdotal evidence indicates that RTW is challenging and requires a range of strategies and supports. This paper aims to present perspectives of those involved in the return to work (RTW) process, with a particular focus on the experiences of three individuals with acquired brain injury (ABI) who have returned to some form of work or meaningful activity, or are in the process of doing so. Perspectives of two Disability Employment Service (DES) providers will also be presented.

Method: Participants took part in informal semi-structured interviews (1:1 and group) to explore their experiences of the RTW process and their thoughts about ways to connect and collaborate with key stakeholders. Key themes were identified for each participant's perspective.

Results: Participants' responses pointed to a number of facilitators and barriers in the process, including strategies to assist preparation and liaison with employers as well as in-situ strategies, such as selecting suitable support people and planning around fatigue. Findings will be presented with regard to key facilitators for the RTW process from the perspective of those with ABI and DES providers.

Conclusion: Return to work or some form of meaningful vocational activity is a valued aim for individuals after ABI. It is important to incorporate the perspective of those with $\mathrm{ABI}$ and associated service providers to ensure productive collaboration and connection with the aim of optimal participation outcomes. 


\section{The Experience of Return to Work in Individuals with Traumatic Brain Injury}

Lauren Libeson, ${ }^{1}$ Marina Downing, ${ }^{1,2}$ Pamela Ross, ${ }^{3}$ Jennie Ponsford, ${ }^{1,2}$ and Marina Downing ${ }^{2}$

${ }^{1}$ School of Psychological Sciences, Monash University

${ }^{2}$ Monash-Epworth Rehabilitation Research Centre

${ }^{3}$ Epworth Healthcare, Richmond

$\boldsymbol{R}$ ackground and aims: Traumatic Brain Injury (TBI) is a leading cause $\mathcal{B}$ of disability in young people, with return to work (RTW) a major goal of recovery. This study aimed to understand the RTW experience of individuals with TBI who had received comprehensive TBI vocational rehabilitation, and to identify the facilitating and limiting factors in the RTW process.

Method: 15 individuals with TBI were interviewed using a semi-structured interview developed by the research team. Interviews were transcribed and thematically analysed to identify common factors and themes.

Results: Three key factors were identified: client-related, work-related and rehabilitation-related. Across these factors, 12 themes that were critical to the success or failure of the RTW programme were identified. Themes within client-related factors included family and social support, cognitive difficulties and motivation to RTW, with returning to work too early associated with unfavourable outcomes. Themes within work-related factors included work modifications, employer support, employee loyalty and financial incentives. Themes within rehabilitation-related factors included the RTW programme, the role of the occupational therapist and work preparation. These key factors impacted the RTW outcome, which comprised three further themes: participants' level of satisfaction with their roles, future vocational outlook and quality of life.

Conclusions: Consideration of these factors can inform improved vocational rehabilitation programmes and may ultimately improve employment outcomes for those with TBI.

\section{A Scoping Study Examining Vocational Rehabilitation in Early Acquired Brain Injury Rehabilitation}

Alena Murray, ${ }^{1}$ Kerrin Watter, ${ }^{1,2}$ Mandy Nielsen, ${ }^{1,2}$ and Areti Kennedy ${ }^{1,2}$

${ }^{1}$ Acquired Brain Injury Transitional Rehabilitation Service, Division of Rehabilitation, Metro South Health, Brisbane, Australia

${ }^{2}$ The Hopkins Centre, Division of Rehabilitation Metro South Health and Griffith University, Brisbane, Australia

$\boldsymbol{B}$ ackground and objectives: Following acquired brain injury (ABI), productive roles (paid/voluntary work or study) are often disrupted. A productive role is related to better health status, greater social integration, and better quality of life. The importance of appropriate, coordinated vocational programmes for adults with disability (including ABI) is recognised in Queensland and beyond. No current vocational rehabilitation (VR) service or framework exists within Queensland Health, therefore, the primary objective of the study was to inform development of an evidence-based model of care for delivering community VR for adults with ABI.

Method: This study uses a mixed-methods approach. A scoping study was performed to examine the extent, range and nature of VR in ABI in Queensland. This included investigating frameworks and identifying elements of VR, conducting a literature review, and identifying gaps in existing rehabilitation services and frameworks for people with ABI, particularly in Queensland. 
Views and experiences of service users and expert clinicians were collected via qualitative semi-structured interviews and focus groups, and analysed using qualitative content analysis. This data led to the development of a VR framework.

Results: A VR framework for early community ABI rehabilitation was developed, and will be presented. The model involves different client vocational 'streams', and can be used to assist in planning VR with onward services. Early clinical data will be presented.

Conclusions: We have created a framework for early community VR that is evidence-based and able to be implemented by a community rehabilitation team.

\title{
Refining the Care and Needs Scale (CANS) to Measure Support Needs in Adults After Traumatic Brain Injury
}

Robyn Tate, Donna Wakim, and Ulli Rosenkoetter

John Walsh Centre for Rehabilitation Research, Northern Clinical School, University of Sydney

\begin{abstract}
$B$ ackground: In spite of hundreds of instruments available to measure function after traumatic brain injury (TBI), few measure environmental factors, even though this exerts a major influence on outcome. The Care and Needs Scale (CANS) is one such instrument, being an eight-category hierarchical scale (Level 0 to Level 7) that uses a 24 -item needs checklist to identify the type and intensity of support need. It has been observed that a large proportion of people with severe traumatic brain injury (TBI) are classified at CANS Level 4 (support for 1-11 hours/day). Accordingly, the aim of the study was to determine the optimal way to subdivide Level 4.

Methods: Eight models were developed within four broad domains: (i) number and (ii) type of activities requiring support, (iii) support services and (iv) number of care hours required. Using archival data, the models were evaluated against eight validating variables.

Results: Forty-seven cases met selection criteria. The models were tested using nonparametric analyses. The best performing model made a tripartite subdivision using type of support: instrumental activities of daily living (ADL), versus instrumental + basic ADL, versus instrumental + basic ADL + high level needs. The total score on the Functional Independence Measure showed significant differences among the respective subgroups $(M=112.5$ vs. 108.0 vs. $98.6 ; z=11.87, p=.003$ ).

Conclusions: The CANS is still configured as an 8-level scale, but Level 4 is now subdivided (4.1, 4.2 and 4.3) on the basis of the type of support need required. We believe that this refined scale will be better able to capture support needs.
\end{abstract}

\section{A Day in the Life of Parents with Physical and Cognitive Deficits}

\author{
Evelina Pituch, ${ }^{1,2}$ Veronique Faguy, ${ }^{3}$ Veronique Gilbert, ${ }^{3}$ Cathy Samson, ${ }^{3}$ Aysha \\ Dominique, ${ }^{3}$ and Carolina Bottari ${ }^{1,2}$ \\ ${ }^{1}$ Centre for Interdisciplinary Research in Rehabilitation of Greater Montreal (CRIR), Montreal, Canada \\ ${ }^{2}$ School of Rehabilitation, Université de Montréal, Montreal, Canada \\ ${ }^{3}$ CIUSSS Centre-Sud-de-l'Ille-de-Montréal - Institut universitaire sur la réadaptation en déficience \\ physique de Montreal, Montreal, Canada
}

ackground and aims: Parenting is one of the most invaluable social
experiences of a lifetime, yet rehabilitation services are little oriented
towards learning to assume a parenting role following a cognitive injury. 
Exceptions do exist, as a Canadian parenting clinic 'Parents Plus' specialises in offering tailored occupational therapy services to parents living with motor or neurological impairments. The aims of this case report were to assess everyday life difficulties encountered by parents with reported cognitive deficits and to identify possible clinical rehabilitation interventions.

Method: A modified version of the Activities of Daily Living (ADL) Profile was used to assess in-home independence performance associated with infant parenting skills. Assessments were video recorded for in-depth analysis and conducted in dyads with a trained occupational therapist and a researcher specialised in cognitive rehabilitation.

Results: Two non-related parents, a father and a mother with very different cognitive injuries and life experiences, were individually assessed during four continuous hours. The first participant, a 46-year-old married father of four children with his youngest being five-month old, had a right haemorrhagic stroke. The second participant, a 30-year-old single mother of a 11month baby had a hypoxic encephalopathy and used a manual wheelchair. Both participants demonstrated repercussions of executive function deficits with regards to planning, carrying out parenting tasks and verifying goal attainments.

Conclusions: Difficulties in parenting tasks and relevant interventions were made explicit using the modified ADL Profile. The dyadic format of assessments abolished most clinical-research barriers and allowed direct translation of research findings to clinical practice.

\title{
Developing Technology to Facilitate Meal Preparation After a Traumatic Brain Injury: Potential Users and Their Caregivers Express Their Needs
}

\author{
Érika Dubuc, ${ }^{1,2}$ Mireille Gagnon-Roy, ${ }^{1,2}$ Mélanie Couture, ${ }^{3,4}$; Nathalie Bier, ${ }^{1,5}$ and \\ Carolina Bottari ${ }^{1,2}$ \\ ${ }^{1}$ School of Rehabilitation, Université de Montréal, Montreal, Canada \\ ${ }^{2}$ Center for Interdisciplinary Research in Rehabilitation, Montreal, Canada \\ ${ }^{3}$ Centre for Research and Expertise in Social Gerontology (CREGÉS), Montreal, Canada \\ ${ }^{4}$ Department of psychology, Universite de Sherbrooke, Sherbrooke, Canada \\ ${ }^{5}$ Centre de recherche de l'Institut Universitaire de Gériatrie de Montréal (CRIUGM), Montreal, \\ Canada
}

$\boldsymbol{B}$ ackground and objectives: Following a traumatic brain injury (TBI), B meal preparation can become challenging. Assistive technologies, such as the cooking assistant $\mathrm{COOK}$, are promising interventions to ensure safety and optimise independence. To facilitate the development and eventual integration of such technologies, a better understanding of the needs of individuals with TBI during meal preparation is needed. This study aimed to explore the perspective of TBI individuals and their caregivers regarding unmet needs and difficulties experienced during meal preparation within their home.

Method: Individuals interviews (TBI individuals, $n=5$ ) and a focus group (caregivers, $n=4$ ) were completed and analysed using content analysis according to the approach of Miles, Huberman and Saldana (2013).

Results: In total, 37 unmet needs and difficulties were identified by participants, and regrouped in seven categories. Two categories emerged as important since they directly interfere with engagement in meal preparation: (1) incongruence with expressed desires and (2) incompatibility of the task complexity with cognitive abilities, energy level and knowledge. Having to adapt the task to their cognitive abilities and knowledge was identified as an obstacle to the task pursue. Finally, findings revealed that lack of motivation, low congru- 
ence with desires and poor ability to adapt the task were associated with less involvement in meal preparation.

Conclusion: This study is a step towards a better understanding of the needs of TBI individuals living in the community regarding meal preparation. Development of technological interventions adapted to the needs of potential users should optimise their acceptability within the home environment.

\title{
Conception with and for Traumatic Brain Injury Persons of an Assistive Technology for Meals Preparation and Exploration of the Usability
}

\author{
Stéphanie Pinard, ${ }^{1,2,3}$ Sylvain Giroux, ${ }^{4}$ Carolina Bottari, ${ }^{1,3}$ Catherine Laliberté, ${ }^{4}$ \\ Hélène Pigot, ${ }^{4}$ Mélanie Couture ${ }^{5}$ Marisnel Carolina Olivares Amaro, ${ }^{4}$ Pierre-Yves \\ Groussard, ${ }^{4}$ and Nathalie Bier, ${ }^{1,2}$ \\ ${ }^{1}$ University of Montreal, Montreal, Canada \\ ${ }^{2}$ Research Center of the University Institute of Geriatrics of Montreal, Montreal, Canada \\ ${ }^{3}$ Centre for Interdisciplinary Research in Rehabilitation of Greater Montreal, Montreal, Canada \\ ${ }^{4}$ DOMUS laboratory, University of Sherbrooke, Sherbrooke, Canada \\ ${ }^{5}$ Center for Research and Expertise in Social Gerontology, University of Sherbrooke, Sherbrooke, \\ Canada
}

Ttroduction: In Canada, 100,000 people sustain a traumatic brain injury (TBI) each year. Young adults with TBI will live an average of 50 years with the physical and cognitive sequelae of their TBI. Approximately $10 \%$ of people with severe TBI reside in long-term care centres not adapted to their needs. Indeed, these residences offer some leisure activities, but no opportunities are provided to prepare one's own meals despite the importance of this activity.

Methodology: As part of an interdisciplinary project, combining the expertise of rehabilitation and computer sciences, COOK was designed with and for people with severe TBI living in a specialised long-term care residence adapted to their needs. COOK is an assistive technology for cognition designed to facilitate meal preparation. User-cantered design was used and all future users participated $(n=3)$ in the process. The design documented the needs of residents and these needs were then combined to evidence-based cognitive rehabilitation practices and transformed into the technological features of COOK. COOK was installed in the three residents' apartments to explore its usability over the short and long term (1, 3 and 6 months post-implementation). Results: COOK consists of two main systems: one to support the functioning (detect errors and offer assistance) and the second to ensure safety (alert staff when high-risk situations occur). All participants were able to prepare meals safely with COOK in their own apartments.

Conclusion: This technology appears promising for the rehabilitation and home support of clients with cognitive disabilities. 


\title{
The Impact of a Driving Cessation Program on Community Participation Goals Following Complex Trauma Injury: A Randomised Controlled Trial of the CarFreeMe TI Program
}

\author{
Stacey George, ${ }^{1,2}$ Chris Barr, ${ }^{1}$ Naomi Jarvis, ${ }^{4}$ Sharron Neeson, ${ }^{5}$ Amy Nussio, ${ }^{1,2}$ \\ and Jacki Liddle ${ }^{3}$ \\ ${ }^{1}$ Clinical Rehabilitation, College of Nursing and Health Sciences, Flinders University, Adelaide, \\ Australia \\ ${ }^{2}$ Occupational Therapy Department, Division of Rehabilitation, Aged Care and Palliative Care, South- \\ ern Adelaide Local Health Network, Adelaide, Australia \\ ${ }^{3}$ School of Information Technology and Electrical Engineering, The University of Queensland, Bris- \\ bane, Australia \\ ${ }^{4}$ Allied Health Services, Brain Injury South Australia (BISA), Adelaide, Australia \\ ${ }^{5}$ Support Services, Paraquad South Australia (PQSA), Adelaide, Australia
}

$\boldsymbol{B}$ ackground and aims: To explore the effectiveness of an education and $B$ support programme (CarFreeMe TI) in addressing community participation goals for individuals who are unable to return to full driving following a trauma injury.

Method: A total of 20 participants (14 male) with a mean age 53.6 (SD 14.3) years old took part in a randomised crossover study. The participants predominantly presented with a principal diagnosis of traumatic brain injury $(n=17)$, and were a mean 13.75 (SD 15.9) years since injury. Individual transport and lifestyle goals were identified and goal performance and satisfaction was rated pre- and post-intervention using a modified Canadian Occupational Performance Measure (COPM). The CarFreeMe TI intervention involved six group sessions with adaptable content relevant to the emotional and practical implications of driving cessation. Guest speakers were invited from relevant community groups to facilitate opportunities for ongoing community participation.

Results: A total of 34 goals were set by 16 participants. Performance on goals increased from 3.65 (SD 2.9) before the intervention to 7.1 (SD2.1) after the intervention, while satisfaction increased from 4.6 (SD 2.8) to 7.5 (SD 1.7) (both $p<.001$ ). Common themes in participant goals were also identified.

Conclusions: A statistically significant improvement in individualised programme goal ratings is consistent with the flexible design of the intervention and the capacity to adapt group content and offer additional individual sessions where appropriate. 
Session 9: Pharmacological and Behavioural Considerations in Rehabilitation

\title{
Developing a Clinical Guideline for the Pharmacological Management of Neurobehavioural Symptoms After Traumatic Brain Injury
}

\author{
Malcolm Hopwood, ${ }^{1,2}$ L. Ponsford, ${ }^{3}$ Mahesh Jayaram,, ${ }^{1}$ Fiona J. Clay, ${ }^{1,4}$; Rachel \\ Batty, ${ }^{1}$ and Amelia J. Hicks ${ }^{3}$ \\ ${ }^{1}$ Department of Psychiatry, University of Melbourne, Melbourne, Australia \\ ${ }^{2}$ Professorial Psychiatry Unit Albert Road Clinic, Department of Psychiatry, University of Melbourne, \\ Melbourne, Australia \\ ${ }^{3}$ Monash-Epworth Rehabilitation Research Centre, Monash Institute of Cognitive and Clinical Neu- \\ rosciences, School of Psychological Sciences, Monash University, Melbourne, Australia \\ ${ }^{4}$ The Australian Centre for Evidence-Based Primary Health Care, Community Care: A Joanna Briggs \\ Institute Centre of Excellence, Adelaide, Australia
}

ackground and aims: Neurobehavioural symptoms (NBS) are common
following traumatic brain injury (TBI) and contribute heavily to impaired
quality of life, cost of care and carer burden. Pharmacotherapeutic management
of NBS is inconsistent and frequently inadequate. The aim of this guideline
is to better inform the use of medications for the treatment and ongoing
management of NBS post-TBI.
Methods: An evidence review of all English language publications before
May 2017 across six bibliographic databases and clinical trials registries was
conducted. NBS covered apathy, anxiety, depression, impulsivity, psychosis,
bipolar disorder, agitation and aggression, NBS during post traumatic amnesia,
pseudo-bulbar affect and Kluver-Bucy Syndrome. Development of the guide-
line was overseen by a steering group. A group of experts across Australia
and New Zealand provided input into the research questions and recommen-
dations. A consumer-carer group provided input of the lived experience. An
international advisory group will review the draft document.
Results: Data were extracted into evidence tables from 156 case reports and
65 primary studies. There was a lack of strong evidence from well conducted
randomised controlled trials. Many studies did not report on safety. For the
guideline, the majority of recommendations made were consensus based. Re-
search based recommendations were made for amantadine in the treatment of
agitation and aggression. Conclusions: The guideline will cover best practice for the management of NBS following TBI including when to review prescribed medications. An Australian clinical guideline for the management of NBS post-TBI offers the opportunity to educate and upskill prescribers and reduce the suffering postTBI. 


\title{
The Efficacy and Harms of Pharmacological Interventions for Neurobehavioral Symptoms in Post Traumatic Amnesia After Traumatic Brain Injury - Systematic Review
}

\author{
Amelia J. Hicks, ${ }^{1}$ Fiona J. Clay, ${ }^{2,3,4}$ Malcolm Hopwood, ${ }^{2,5}$ Amelia C. James, ${ }^{1}$; \\ Mahesh Jayaram, ${ }^{2}$ Rachel Batty, ${ }^{2}$ and Jennie L. Ponsford ${ }^{1}$ \\ ${ }^{1}$ Monash-Epworth Rehabilitation Research Centre, Monash Institute of Cognitive and Clinical Neu- \\ rosciences, School of Psychological Sciences, Monash University, Melbourne, Australia \\ ${ }^{2}$ Department of Psychiatry, University of Melbourne, Melbourne, Australia \\ ${ }^{3}$ Department of Forensic Medicine, Monash University, Southbank, Australia \\ ${ }^{4}$ The Australian Centre for Evidence-Based Primary Health Care, Community Care: A Joanna Briggs \\ Institute Centre of Excellence, Adelaide, Australia \\ ${ }^{5}$ Professorial Psychiatry Unit Albert Road Clinic, Department of Psychiatry, University of Melbourne, \\ Melbourne, Australia
}

\begin{abstract}
$\boldsymbol{R}$ ackground and aims: Many individuals in the post-traumatic amnesia (PTA) period following traumatic brain injury (TBI) experience significant neurobehavioral symptoms (NBS), including agitation, aggression and disorientation. These symptoms are often associated with increased length of stay and low engagement in rehabilitation, self-inflicted harm and risks of physical violence. The aim of this systematic review was to critically evaluate the evidence on pharmacological interventions for NBS in PTA following TBI in adults.

Methods: We reviewed studies in English, published before May 2017. MEDLINE, PubMed, CINAHL, EMBASE, PsycINFO and CENTRAL databases were searched, with additional hand searching of key journals, clinical trials registries and international drug regulators. Evidence quality was assessed using the Joanna Briggs Institute Critical Appraisal Instruments.

Results: Thirteen studies were identified, including three randomised controlled trials, three cohort studies, and seven case series. Pharmacological interventions were complex and varied, and often lacked detailed descriptions of dose, frequency and duration of the pharmacological intervention. The three higher quality studies examined the efficacy of rosuvastatin, sertraline and amantadine. Neither amantadine nor sertraline had an effect on NBS. With respect to harms and adverse events, there was an increase in creatinine for one patient administered rosuvastatin, and no adverse events for patients receiving amantadine. Neither sertraline nor amantadine had an effect on PTA duration, and the effects of rosuvastatin were unclear.

Conclusions: There is a paucity of well powered RCT studies of pharmacological interventions for NBS in PTA, and more research is needed to provide evidence-based treatment recommendations.
\end{abstract}

\section{Agitated Behaviour, Therapy Participation and Activities of Daily Living Retraining During Posttraumatic Amnesia}

\author{
Jessica Trevena-Peters, ${ }^{1,2}$ Jennie Ponsford, ${ }^{1,2}$ and Adam McKay ${ }^{1,2,3}$ \\ ${ }^{1}$ Monash Institute of Cognitive \& Clinical Neurosciences and School of Psychological Sciences \\ Monash University, Clayton VIC, Australia \\ ${ }^{2}$ Monash Epworth Rehabilitation Research Centre, Richmond VIC, Australia \\ ${ }^{3}$ Epworth Healthcare, Richmond VIC, Australia
}

$\boldsymbol{R}$ ackground and aims: Patients in posttraumatic amnesia (PTA) may $B$ receive limited rehabilitation due to the risk of overstimulation and agitation. This assumption has not been tested. The study aimed to examine the relationship between agitated behaviour and participation in activities of daily 
living (ADL) retraining while in PTA. Additionally, strategies used to engage individuals during PTA, while agitated and confused, are discussed.

Method: 104 participants with severe traumatic brain injury, admitted to inpatient rehabilitation, in PTA $>7$ days randomised to receive treatment as usual (TAU) with daily ADL retraining (treatment), or TAU alone (physiotherapy, necessary speech therapy), during PTA. ADL retraining during PTA followed errorless and procedural learning principles and the manual included strategies for therapy engagement during PTA. Primary outcomes were agitation (Agitated Behaviour Scale) and therapy participation (minutes and missed sessions). Regression modelling was conducted for between group comparison of agitation data (treatment versus TAU) and the longitudinal relationship between agitation and therapy participation (treatment condition).

Results: There were no group differences in agitated behaviour (average scores, peak scores or number of clinically agitated days) between treatment and TAU groups. For treated patients, there was no significant relationship between agitation and therapy participation (therapy minutes or missed ADL treatment sessions). Strategies for engaging agitated and confused individuals in PTA appeared beneficial.

Conclusion: This study demonstrated that agitation is not increased by delivery of structured ADL retraining during PTA, and agitation did not limit therapy participation. This supports consideration of active therapy during PTA and that this is possible, despite agitated behaviour.

\title{
Heart Rate Variability Biofeedback: Can It be Used to Regulate Emotion for Individuals with Traumatic Brain Injury?
}

\author{
Travis Wearne, ${ }^{1,2}$ Emily Trimmer,,2 Emily Wilson,, ${ }^{1,2}$ Poss Logan,, ${ }^{1,2}$ Heather \\ Francis, ${ }^{1,2}$ Jacqueline Rushby, ${ }^{1,2}$ and Skye McDonald ${ }^{1,2}$ \\ ${ }^{1}$ Department of Psychology, University of New South Wales, Sydney, Australia \\ ${ }^{2}$ Centre for Research Excellence in Brain Recovery, Australia
}

$\boldsymbol{B}$ ackground and aims: While difficulties regulating emotion are almost $B$ ubiquitous after traumatic brain injury (TBI), remediation techniques are limited. Heart-rate variability (HRV) is a physiological measure of an individual's response to environmental demands and can be modified using biofeedback training. It is not known, however, whether biofeedback can be used to regulate emotions or whether it is a feasible technique following TBI. In these studies, we investigated whether biofeedback can mediate response to anger-inducing stimuli in healthy controls and examined the feasibility of HRV biofeedback for individuals with TBI.

Method: In experiment 1, participants received HRV biofeedback $(n=29)$ or control $(n=29)$ and measures of HRV were recorded during baseline, biofeedback training and anger induction. In experiment 2, HRV was recorded in 30 individuals with severe TBI and 30 controls at rest and during a single session of biofeedback.

Results: In experiment 1, the biofeedback group had higher HRV than active controls during both training and anger induction, with HRV associated with self-reported emotional response for participants receiving biofeedback but not for controls. In experiment 2, both TBI and controls showed increased HRV during biofeedback compared to baseline.

Conclusion: HRV can be used as an index of emotion regulation, specifically anger, and that HRV biofeedback can improve objective and subjective response to anger-inducing stimuli. Given individuals with TBI and controls respond similarly to biofeedback, this may represent a novel technique for 
mediating emotional difficulties following injury. Results from our subsequent multiple treatment trial will also be discussed.

\title{
Understanding Comorbidities of People with an Acquired Brain Injury to Inform Treatment Decisions
}

\author{
Elly Williams, and Angelita Martini \\ ${ }^{1}$ Brightwater Research Centre, Brightwater Care Group, Perth, Australia
}

$\boldsymbol{R}$ ackground and objectives: People with an acquired brain injury (ABI) $\mathcal{B}$ often experience dual diagnosis and the presence of other disorders, impacting treatment decisions. Comorbidities of people with ABI may have existed pre-injury, be a result of injury, or been a factor in causing injury. It is therefore important to consider the comorbidities of people with ABI to ensure the best rehabilitation outcomes and enable successful reintegration into the community. The objective of this study was to determine the comorbidities of clients with an $\mathrm{ABI}$ on admission to a slow-stream, post-acute rehabilitation service.

Method: This is a retrospective, observational cohort study of $n=232$ participants with an ABI who were admitted to a post-acute rehabilitation service from June 2009 to September 2017. Comorbidity data was collected on admission to the service, stratified by age and analysed using STATA statistical software.

Results: Of all clients $64 \%$ had one or more comorbidity. Of these, $33 \%$ had a mental health disorder (Depression, Anxiety, Bipolar, PTSD and schizophrenia), and $26 \%$ a comorbidity of depression. Other main comorbidities were, hypertension (18\%), diabetes (14\%), epilepsy (9\%) and alcohol abuse (8\%). Significantly, when stratifying for age, all (100\%) of the clients aged 17-29 with a comorbidity (47\%) had a mental health comorbidity. This was lower in the older age-groups, ranging from 29-50\%.

Conclusions: Rehabilitation from an ABI is complex as clients often have comorbidities. It is important for health services to consider these comorbidities in planning treatment and rehabilitation services, particularly for mental health disorders and chronic diseases.

\section{The Lived Experience of Health Professionals Working with Patients with a Disorder of Consciousness (DOC) in a Sub-Acute Acquired Brain Injury Unit}

Evelyn Harkins, Delwyne Stephens, and Eric Cruz

Alfred Health, Acquired Brain Injury (ABI) Unit, Melbourne, Australia

$B$ ackground and objectives: Working with patients with a disorder of consciousness (DOC) is a specialised area of rehabilitation; patients are complex, have uncertain outcomes and highly stressed families. Some literature points to professional burnout, emotional exhaustion and stress when working with the catastrophically brain injured, but there is limited research into how health professionals experience their encounters with patients with a DOC. The aim of this project is to consider the lived experience of health professionals working with patients with DOC in a sub-acute acquired brain injury (ABI) setting so that support mechanisms for staff can be considered.

Method: In-depth, semi-structured interviews $(n=11)$ were completed with a convenience sample of Health Professionals from different disciplines working in the Alfred Health ABI Unit. Thematic analysis was undertaken. 
Results: Analysis revealed staff experiences to be both highly challenging and highly rewarding. Prominent themes include (1) the importance of an interdisciplinary team and working with other staff who have expertise (2) the intricacies of working with families (3) the benefit of having DOC work instructions (4) complexity of the ABI unit, separate to the patient with DOC and (5) the need for formal and informal debriefing.

Conclusions: Working with patients with DOC needs to be considered in the context of these patients being one of many in a highly complex clinical environment. Health professionals working with patients with a DOC specifically identify expertise within the team, formal and informal support processes, and formal work instructions as positive factors in supporting them to undertake this highly challenging and emotional work.

\section{Positive Behaviour Support (PBS) Following Brain Injury: What, Why, and How?}

Alinka Fisher

Disability and Community Inclusion, College of Nursing and Health Sciences, Flinders University, Adelaide, Australia

$R$ ackground and aims: Positive behaviour support (PBS) is gaining increasing attention, with a number of intervention experiments demonstrating its effectiveness for individuals with brain injury. However, it seems the foundations of PBS are often misunderstood, which could result in interventions that are not consistent with its theory and practices. The aim of this review was to provide a current perspective regarding the PBS in the context of brain injury, which is important in informing evidence-based practices.

Methods: Literature review

Results: PBS has evolved from the science of Applied Behaviour Analysis (ABA), and is based on principles of person-centred care and normalisation. The primary goal of PBS is to improve an individual's quality of life through (1) functional behaviour assessment and analysis (2) skill development, (3) environmental modification and (4) contingency strategies. However, PBS interventions for individuals with brain injury often emphasise antecedent-based strategies, as individuals may have difficulties learning from the consequences of his or her behaviour. There is increasing evidence supporting PBS interventions following brain injury; however, a majority of PBS studies have been conducted in institutional or residential environments, rather than unstructured community settings.

Conclusions: PBS provides a comprehensive framework for supporting individuals with brain injury who present with behaviours of concern. In implementing evidence-based practices, it is important for interventions to be true to the theory and practices of PBS, and that these clinical approaches are transferred to community-based settings.

\section{Recommendations for Working with People with a Diagnosis of Dementia: The Importance of What Comes After Guideline Development}

Michelle Kelly, Jayde Noble, and Luke Brock

Centre for Brain and Mental Health Research, School of Psychology, University of Newcastle, Newcastle, Australia

$\boldsymbol{R}$ ackground and objectives: Dementia is the second leading cause of death in Australia, with an estimated 420,000 people living with condition, and expectations that this number will continue to rise. Corresponding to the growth in the incidence of dementia, is the detrimental impact on the 
psychosocial wellbeing of those with the diagnosis, and their family and informal carers. In response to the growing need for support in the provision of care to people with dementia, the NHMRC endorsed Australian Clinical Practice Guidelines were released in 2016. It is not yet clear whether the guidelines have been successfully disseminated and implemented.

Method: Using an on-line platform, 110 medical and allied health clinicians working with people with dementia completed a 76-item survey to determine level of awareness of, and adherence to these guidelines, as well as factors affecting adherence rates. Of these respondents, approximately 60 were eligible for inclusion in analyses.

Results: The majority of respondents was female (90\%), psychologists (51\%), with postgraduate training $(65 \%)$ and had greater than four years' experience working in dementia care (77\%). As expected, those clinicians who were aware of the guidelines had significantly higher adherence rates than those who were not aware $(t(38)=2.4, p=.02)$. Adherence was not found to be dependent on post-graduate education or professional development ( $p s>.05$ ). Data collection is ongoing.

Conclusions: Preliminary results suggest further dissemination and implementation strategies are needed to increase awareness of these guidelines, and in turn, influence uptake in clinical practice.

\title{
Session 10: Working with People and their Communication
}

\section{Role of Inferential Language and Processing Style in Discourse Comprehension Following Right Hemisphere Stroke}

\author{
Petrea Cornwell, ${ }^{1,2,3}$ Ronelle Hewetson, ${ }^{1,4}$ and David Shum ${ }^{1,4}$ \\ ${ }^{1}$ Menzies Health Institute Queensland, Griffith University, Gold Coast, Australia \\ ${ }^{2}$ School of Allied Health Sciences, Griffith University, Gold Coast, Australia \\ ${ }^{3}$ Allied Health Research Collaborative, The Prince Charles Hospital, Brisbane, Australia \\ ${ }^{4}$ School of Applied Psychology, Griffith University, Brisbane, Australia
}

$\boldsymbol{B}$ ackground and objectives: Discourse comprehension deficits contribute 3 to communication breakdowns in individuals with right hemisphere (RH) stroke. Comprehension deficits have been attributed to difficulty interpreting inferential language, para- / extra-linguistic content, or style of processing, with less emphasis on syntactic structures. This study aimed to provide a comprehensive evaluation of factors contributing to discourse comprehension abilities after RH stroke exploring effect of syntactic processing, main/detailed or implied/stated content, and inferential language.

Method: Participants with RH stroke $(n=14)$ and healthy controls $(n=14)$ completed three measures of discourse comprehension, namely sentencepicture matching - auditory version (Psycholinguistic Assessment of Language Processing in Aphasia subtest 55: syntactic processing), The Awareness of Social Inferencing Test (TASIT-R: inferential language), and the Discourse Comprehension Test.

Results: Discourse comprehension abilities were significantly reduced for individuals with RH stroke in comparison to controls $(p=.001)$, in particular difficulty comprehending the main idea $(p=.01)$ or implied content $(p=.04)$. Individuals with RH stroke scored significantly lower on the syntactic processing tasks $(p=.01)$ and comprehension of social inferential language (minimal context: $p=.03$, enriched context: $p<.001$ ) when compared to controls. Correlational analysis revealed comprehension of the main idea in discourse was 
moderately associated with social inferencing (enriched context: $p=.045$ ) for RH stroke individual's.

Conclusions: Findings suggest syntactic processing deficits while evident in individuals with RH stroke are not strongly associated with discourse comprehension abilities, but may add support for processing style and inferential language contributing to impaired discourse comprehension.

\section{Are There Sex Differences in Social Cognition after Acquired Brain Injury?}

Christine Padgett, ${ }^{1}$ George Mikalha, ${ }^{1}$ and Cynthia Honan ${ }^{2}$

${ }^{1}$ School of Medicine (Psychology), University of Tasmania, Hobart

${ }^{2}$ School of Medicine (Psychology), University of Tasmania, Launceston

$\boldsymbol{R}$ ackground and aims: Acquired brain injury (ABI) is often associated with impairments to social cognition. It has also been suggested that in healthy populations, females may outperform males on measures of social cognition, however, there is little exploration of sex differences following ABI. Method: A total of 73 participants $(\mathrm{ABI}=39$, controls $=34$ ) completed a range of social cognition measures; the Social-Emotional Questionnaire (SEQ; Emotional Recognition and Empathy Subscales), the Interpersonal Reactivity Index (IRI - Empathy Subscale) and The Awareness of Social Inference Test, Shortened version (TASIT-S; Parts I, II and III). Informants also completed the informant version of the SEQ.

Results: 2 (male vs. female) x 2 (ABI vs. control) ANOVAs were conducted to compare social cognitive ability on each task. There were no significant differences between $\mathrm{ABI}$ and healthy controls, or sex, on the participant version of the SEQ, however, informants rated ABI participants significantly worse on the SEQ, and males, regardless of group, rated themselves poorer on the IRI scale. There was a significant interaction between sex and group on all TASIT-S subscales, where males with an $\mathrm{ABI}$ performed worse than males without and $\mathrm{ABI}$, or females with or without an ABI. There were no significant differences between females with or without ABI.

Conclusions: Although this study was cross-sectional, the findings suggest that females may protected against deficits to social cognition following ABI, but that differences may only be identifiable by using objective measures such as the TASIT-S. 


\section{The Challenge of Monitoring Fidelity in Trials of Complex Behavioural Interventions: The COMPARE Fidelity Protocol and Progress Results}

Marcella Carragher, ${ }^{1}$ John E. Pierce,${ }^{1,2}$ Lyndsey Nickels, ${ }^{3}$ Leanne Togher, ${ }^{4}$ Marcus Meinzer, ${ }^{5}$ Tapan Rai, ${ }^{6}$ Erin Godecke, ${ }^{7}$ Joosup Kim, ${ }^{8,9}$ Dominique A. Cadilhac, ${ }^{8,9}$ Abby Foster, ${ }^{10}$ Melanie Hurley, ${ }^{1}$ David Copland, ${ }^{5}$ and Miranda Rose ${ }^{1}$

${ }^{1}$ School of Allied Health, La Trobe University, Melbourne, Australia

${ }^{2}$ Speech Pathology, Cabrini Health, Melbourne, Australia

${ }^{3}$ Cognitive Science Department, Macquarie University, Sydney, New South Wales, Australia

${ }^{4}$ Faculty of Health Sciences, University of Sydney, Sydney, Australia

${ }^{5}$ UQ Centre for Clinical Research, University of Queensland, Brisbane, Queensland, Australia

${ }^{6}$ School of Mathematical and Physical Sciences, University of Technology Sydney, Sydney, New South Wales, Australia

${ }^{7}$ School of Psychology and Social Sciences, Edith Cowan University, Joondalup, Western Australia, Australia

${ }^{8}$ School of Clinical Sciences at Monash Health, Monash University, Clayton, Victoria, Australia ${ }^{9}$ Stroke Division, Florey Institute of Neuroscience and Mental Health, Heidelberg, Vic Australia

${ }^{10}$ Speech Pathology, Monash Health, Melbourne, Australia

$\boldsymbol{B}$ ackground and objectives: Treatment fidelity is a complex evaluative process that forms a critical aspect of all health-related intervention studies. Its objective is to determine whether an intervention was delivered as intended and report how this was monitored. Evidence of a high level of treatment fidelity is an important benchmark of quality, signalling confidence in the findings of the study and potential for implementation. Despite its benefits, treatment fidelity has been inconsistently monitored and reported in behavioural intervention studies.

Method: The COMPARE study is an Australian-based three-arm randomised controlled trial (planned sample size $n=216$ ) which aims to determine whether two novel, intensive and contrasting treatments for chronic post-stroke aphasia are superior to usual care. Based on the theoretical models underlying the trial interventions, we developed a fidelity protocol to monitor and enhance treatment delivery by multiple clinicians across Australia. The fidelity protocol adheres to the recommended standards outlined in the TiDIER statement (a template for reporting interventions stemming from the CONSORT 2010 and SPIRIT 2013 statements).

Results: The COMPARE fidelity protocol is currently being implemented. An overview of the protocol will be presented, including the theoretical rationale, methods, challenges and results to date. Data will be presented on the assessment measures, including (a) standardised clinician training, (b) video recording all assessment and treatment sessions, (c) providing feedback in real-time to clinicians and (d) monitoring for therapist drift.

Conclusions: The COMPARE fidelity protocol offers one solution to the challenge of monitoring and enhancing treatment fidelity within a behavioural change intervention. 


\title{
Experiences of People with Aphasia and Their Spouses Involved in An Interdisciplinary Community Aphasia Group
}

\author{
Michelle Attard, ${ }^{1}$ Leanne Togher, ${ }^{2}$ Yasmine Loupis, ${ }^{3}$ and Miranda Rose ${ }^{1}$ \\ ${ }^{1}$ Discipline of Speech Pathology, La Trobe University, Melbourne, Australia \\ ${ }^{2}$ Faculty of Health Sciences, The University of Sydney, Sydney, Australia \\ ${ }^{3}$ Social work, St Vincent's Hospital, Sydney, Australia
}

\begin{abstract}
$B$ ackground \& objective: Research investigating the specific mechanisms and processes within successful community aphasia groups (CAGs) is limited, with a small amount of research addressing the consumer experience. We sought to understand member experiences of attending a 2-hour $\times 12$ week speech pathologist- and social worker-led CAG, the Interdisciplinary CAG (InterD-CAG).

Method: We explored the experiences of four people with chronic, severe aphasia and their spouses regarding their attendance at the InterD-CAG. We conducted semi-structured interviews and analysed the data using Framework Analysis.

Results: Members shared their preferences for programme activities and group format, with some experiences leading to suggestions for improving the model. The group dynamics and environment were considered positive overall, and members highlighted a range of positive impacts for both themselves and their spouse. Together, these elements informed members' evaluation that the model contained something for everyone, but may not be a 'one stop shop' given ongoing needs for therapy. Overarching themes for spouses relate to the group in the context of a research trial and notions of therapy and recovery.

Conclusions: The InterD-CAG resulted in a range of positive outcomes overall for both members with aphasia and spouses, with members perceiving value in the model of service. Implications for CAG research and service delivery in chronic aphasia rehabilitation will be discussed.
\end{abstract}

\section{Experiences of Staff Involved in an Interdisciplinary Community Aphasia Group}

Michelle Attard, ${ }^{1}$ Leanne Togher, ${ }^{2}$ Yasmine Loupis, ${ }^{3}$ and Miranda Rose ${ }^{1}$

${ }^{7}$ Discipline of Speech Pathology, La Trobe University, Melbourne, Australia

${ }^{2}$ Faculty of Health Sciences, The University of Sydney, Sydney, Australia

${ }^{3}$ Social work, St Vincent's Hospital, Sydney, Australia

$\boldsymbol{R}$ ackground and objective: Research investigating the specific mechanisms and processes within successful community aphasia groups (CAGs) is limited. Few studies address the experience of facilitators yet this data is vital for improving staff training and maintaining positive contribution during CAGS. We sought to understand staff experiences of involvement in a 2-hour $\times 12$-week CAG for four people with severe aphasia and their spouses, the Interdisciplinary CAG (InterD-CAG).

Method: We explored the experiences of a social worker, peer with aphasia, and aide regarding their involvement in the InterD-CAG. We conducted semistructured interviews and analysed the data using Framework Analysis.

Results: Staff shared their perceptions of group logistics, including programming, aspects of group composition and the level of programme structure and flexibility. They valued the team and referred to positive qualities impacting their performance and contributions. The group dynamics and environment were considered positive overall, with some aspects of dynamics yielding challenges for staff. Staff also described a range of positive impacts for members and themselves. All of these elements contributed to perceived value regarding 
the InterD-CAG model of service. Overarching themes related to concepts of therapy and recovery, and group service more generally.

Conclusions: The staff's experience reflects the overall acceptability of the InterD-CAG programme. Implications for $\mathrm{CAG}$ research and service delivery in chronic aphasia rehabilitation will be discussed.

\title{
A Systematic Review of Conversational Skills Intervention for Adults with Acquired Brain Injury, Significant Other Reports
}

Leah Paice, Annalle Aleligay, and Martin Checklin

Rehabilitation Speech Pathology and Dietetics, Epworth HealthCare, Melbourne, Australia

$\boldsymbol{R}$ ackground: Deficits in pragmatics and social skills following an acquired 3 brain injury (ABI) have been well documented. These difficulties can profoundly impact on relationships and have a significant effect on family members. However, there are limited studies that consider the significant other as a primary outcome when evaluating interventions.

Aims: To determine the most effective intervention for improving conversation skills in adults following an ABI, from the perspective of a significant other.

Methods: Six electronic databases relevant to speech pathology or brain injury were searched. Grey literature, reference lists and citation indexes were also searched. Studies that met the inclusion and exclusion criteria were screened. Reviewers independently extracted data from full-text reviews and performed bias analysis using the Downs and Black quality checklist.

Results: Six studies were included in the final review; three randomised controlled trials (RCTs), one non-RCT and two of other methodologies. Four studies delivered intervention to the individual with an ABI, one to the communication partner only, and one to both the individual and their communication partner. Intervention programmes ranged from 4 to 12 weeks and from 12 to 48 hours total contact time.

Conclusions: All studies showed improvement in at least one primary or secondary outcome measure. Findings are supportive of intervention to both the person with an $\mathrm{ABI}$ and their communication partner. All studies contained bias and poor compliance with significant other data. There were no consistent trends with setting, length of programmes or intervention recipients. More rigorous studies are needed to investigate these variables.

\section{Early Prediction of Social Participation Following Right Hemisphere Stroke}

\author{
Ronelle Hewetson, ${ }^{1,2,3}$ Ann McGuinness, ${ }^{1}$ Petrea Cornwell, ${ }^{1}$ and David Shum ${ }^{3}$ \\ ${ }^{1}$ School of Allied Health Sciences, Griffith University, Gold Coast, Australia \\ ${ }^{2}$ Metro North Hospital and Health Service, Brisbane, Australia \\ ${ }^{3}$ School of Applied Psychology, Griffith University, Brisbane, Australia
}

$\boldsymbol{B}$ ackground and aims: Cognitive-communication deficits (CCD) follow3 ing right hemisphere $(\mathrm{RH})$ stroke may restrict communication-based participation; however, it is not known who are at particular risk for participation restrictions. This study compared routinely administered acute stroke screens of communication and cognition with future reported social participation.

Method: Patients with first onset RH stroke were randomly selected. Acute admission screening data included Mini-Mental State Examination (MMSE), verbal fluency with phonemic constraint (F-A-S), presence or absence of CCD. Telephone survey, utilising the Sydney Psychosocial Reintegration Scale (SPRS-R), determined social participation change. 
Results: All RH stroke participants ( $n=36, M=65.5$ years of age) were mobile and majority $(n=35)$ discharged home. Social participation change was reported for 35/36. A significant difference in SPRS-R Logit mean score was found between those with $\operatorname{CCD}(M=59.33, S D=10.98)$ and those without $\operatorname{CCD}(M=80.78, S D=14.31 ; t(34)=-5.09, p=.00)$, and for participants with F-A-S scores within normal range $(M=73.91, S D=16.58)$ and F-A-S scores $1.5 S D$ below mean $(M=59.39, S D=11.53 ; t(34)=2.85$, $p=.007)$. The magnitude of differences was large, eta squared $=.432$ and .192 , respectively. No significant difference was found based on MMSE score that $<$ versus $\geq 24$.

Conclusion: Risk profiles of social participation restriction may positively impact timing and focus of rehabilitation. Acute stroke screening of general versus domain specific cognition and communication warrants further exploration in people with RH stroke.

\section{Seeking Optimal Interventions to Enhance Patient-Provider Communication: A Review of the Literature}

Edwina Lamborn, Marcella Carragher, Robyn O'Halloran, and Miranda Rose

Aphasia Lab, School of Allied Health, La Trobe University, Melbourne, Australia

$B$ ackground and objectives: For people with communication disabilities, ineffective communication in healthcare is associated with serious adverse events and negative emotional experiences such as frustration, discomfort and feelings of disempowerment. Interventions are required to enhance patient-provider communication and thus the quality of care and experience of those with communication disabilities. The aim of this literature review was to identify research evidence to inform optimal interventions to enhance communication with people with aphasia in healthcare settings.

Method: The literature explored (i) factors influencing effective communication in healthcare, (ii) interventions to enhance effective patient-provider communication and (iii) issues surrounding implementation of these interventions in healthcare.

Results: Evidence relating to patient-provider communication is distributed widely across the literature. The review identified barriers and facilitators to effective communication in healthcare as well as a number of existing interventions to enhance patient-provider communication. These interventions include augmentative and alternative communication devices and communication partner training, with a potential role of mobile technology to support delivery. An evidence gap exists regarding the effectiveness of interventions on improving communication between health care professionals and people with aphasia and the impact on quality and safety of care. Important themes were identified relating to health professionals, patients, institution/systems and the nature of the intervention.

Conclusions: Effective patient-provider communication could improve quality and safety of care. Interventions are needed to support communication between people with aphasia and the healthcare team yet relevant evidence is widely dispersed. This review's findings will inform the development of optimal interventions to improve patient care. 


\title{
Session 11: Connecting Through Relationships
}

\section{Narrative Storytelling: A Collaborative Therapeutic Relationship}

\author{
Kate D'Cruz, ${ }^{1}$ Jacinta Douglas, ${ }^{1,2}$ and Tanya Serry ${ }^{1}$ \\ ${ }^{1}$ School of Allied Health, La Trobe University, Melbourne, Australia \\ ${ }^{2}$ The Summer Foundation, Melbourne, Australia
}

\begin{abstract}
$B$ ackground and objectives: Narrative storytelling is a relational process between the storyteller and the listener. Despite evidence of the therapeutic value of narrative storytelling, there is limited direct research in the context of acquired brain injury (ABI). The aim of this study was to better understand the experience and impact of narrative storytelling following ABI. This presentation will focus upon the experiences of both the storyteller participants with $\mathrm{ABI}$, and the facilitator participants.

Method: In-depth interviews were conducted with eight adults with severe $\mathrm{ABI}$, who have previously participated in storytelling workshops to produce a personal narrative, and six facilitators of the storytelling. In total, 24 transcripts (17 from participants with ABI, 7 from facilitators) were analysed using constructivist grounded theory methods.

Results: A key finding that emerged from the analysis was the therapeutic experience of narrative storytelling for the storytellers, and the centrality of the storyteller and facilitator relationship to this process. Both storyteller and facilitator participants shared experiences of 'feeling heard', 'releasing emotions' and 'reflecting and learning' as part of the collaborative relationship fostered through the storytelling experience.

Conclusions: The findings of this research provide further evidence to support the therapeutic value of narrative storytelling, emphasising the collaborative relationship between the storyteller and the listener. Further, narrative storytelling offers potential as a person-centred approach to meaningful goal setting and intervention in brain injury rehabilitation.
\end{abstract}

\section{Comparison of Goal Engagement and Outcomes for Individuals with Changes in Self-Awareness After Acquired Brain Injury}

\author{
Sarah Prescott, ${ }^{1}$ Jennifer Fleming, ${ }^{1}$ Emmah Doig, ${ }^{1}$ Nicole Weir ${ }^{2}$ \\ ${ }^{7}$ School of Health and Rehabilitation Sciences, The University of Queensland, Brisbane, Australia \\ ${ }^{2}$ Occupational Therapy Department, Princess Alexandra Hospital
}

R ackground and aims: Changes in self-awareness are common after ac3 quired brain injury (ABI), including impaired self-awareness or hyperawareness of impairments. These changes result in reduced community and social participation and impact on rehabilitation engagement. Understanding how engagement in rehabilitation differs according to changes in self-awareness is therefore necessary, particularly engagement in goal setting. However, no studies have investigated how changes in self-awareness influence engagement in goal setting. Therefore, the aim of this study was to examine engagement in goal setting and goal outcomes of clients with ABI according to their selfawareness.

Method: A prospective cohort design was used to recruit 44 communitydwelling adults with $\mathrm{ABI}$, receiving rehabilitation at an outpatient service or community-based private practices. Data collected were 66 audio-recorded goal setting sessions, questionnaire data collected immediately after goals were set, and goal outcome data twelve weeks later. Variables analysed included: the client-centeredness of goals, motivation for rehabilitation, therapeutic alliance, 
total goal setting time and the percentage of words spoken by the client. Participants were classified into three self-awareness groups: hyper-aware, accurate self-awareness and impaired self-awareness.

Results: Engagement in goal setting and goal outcomes did not differ across the three self-awareness groups, with high levels of client-centeredness of goals and therapeutic alliance in each group.

Conclusions: Participants with changes in self-awareness were as engaged in goal setting to develop and achieve client-centred goals as those without impaired self-awareness. Establishing a strong therapeutic alliance may be necessary to engage clients with changes in self-awareness in goal setting.

\title{
How Does Impaired Self-Awareness Influence Clinicians' Selection of Cognitive Rehabilitation Interventions?
}

\author{
Danielle Sansonetti, ${ }^{1}$ Natasha Lannin, ${ }^{2,3}$ Jacqui Wheatcroft, ${ }^{2}$ Laura De Lacy, ${ }^{1}$ \\ Jenny Fleming, ${ }^{4}$ and Julia Schmidt ${ }^{5}$ \\ ${ }^{7}$ Alfred Health Acquired Brain Injury Rehabilitation Centre, Melbourne, Australia \\ ${ }^{2}$ Occupational Therapy Department, Alfred Health, Melbourne, Australia \\ ${ }^{3}$ School of Allied Health, La Trobe University, Melbourne, Australia \\ ${ }^{4}$ School of Health and Rehabilitation Sciences, University of Queensland, Brisbane, Australia \\ ${ }^{5}$ University of British Columbia, Vancouver, Canada
}

$\boldsymbol{B}$ ackground and objectives: Self-awareness deficits following acquired $\mathcal{B}$ brain injury (ABI) impact on rehabilitation outcomes. Intact selfawareness is believed to be a prerequisite for successful delivery of many cognitive rehabilitation interventions. While several practice frameworks have been recommended to guide cognitive intervention for various stages of awareness, little is known about how therapists evaluate changes in awareness over time and how this influences prescription of cognitive rehabilitation. The aim of this study is to explore the views, beliefs and attitudes of clinicians on selecting and adapting cognitive rehabilitation strategies with patients after ABI who demonstrate impaired self-awareness.

Method: Qualitative focus group study with seven occupational therapists. Stimulus questions explored current approaches to assessment and clinical reasoning, along with how assessment of patients' self-awareness impacts on selection of rehabilitation interventions in an inpatient rehabilitation setting. Results were analysed using a previously developed framework (directed content analysis).

Results: The adaptation of cognitive rehabilitation approaches when working with people with impaired self-awareness was influenced by the following main themes: timing of assessment of self-awareness; validity of assessment results; clinical reasoning around selection of cognitive rehabilitation treatment approaches; and beliefs around the ability for assessment results to predict future function.

Conclusions: This study highlights that selection of a robust measure of selfawareness, and understanding about the efficacy of cognitive rehabilitation approaches in adults with self-awareness deficits is key. Best practice cognitive rehabilitation should include evaluation and re-evaluation of self-awareness over time to ensure that cognitive rehabilitation programmes remain personcentred and targeted towards patients' changing needs. 


\title{
Mastery of Cognitive Rehabilitation Strategies and Changing Care Needs: The Lived Experience of an Individual with Acquired Brain Injury (ABI) in the Community
}

\author{
Simone Wilson, and Danielle Sansonetti \\ Acquired Brain Injury Rehabilitation Centre, Alfred Health, Caulfield, Australia
}

\begin{abstract}
$\boldsymbol{R}$ ackground and objectives: The need for formal supports to achieve community living following an acquired brain injury (ABI) results in considerable financial burden (Lannin et al., 2014). Cognitive rehabilitation is required to support the changing needs, with mastery of cognitive strategies required to minimise dependence on formal supports (Ylvisaker \& Feeney, 1998). This paper aims to explore implementation of cognitive strategies and associated change in care needs over time from the perspective of the patient and their family.

Method: This descriptive case study will outline the process of adaptation of cognitive strategies for a 35 year old man with severe ABI across a three year rehabilitation timeframe. Semi-structured interviews explored the patient and family experiences across the recovery pathway, with qualitative interview data synthesised thematically.

Results: Themes will be presented including: patient and family engagement, awareness and mastery of cognitive strategies, acceptance and positive risktaking. Enablers and barriers to transferring control of strategies from therapist to the patient will also be explored.

Conclusions: Cognitive rehabilitation in ABI is a complex and dynamic process requiring constant evaluation and modification to strategies to meet an individual's changing needs and maximise independence. An integrated and holistic approach that includes active engagement from the patient and significant others is integral to achieving meaningful long term outcomes.
\end{abstract}

\section{Impact of Group Art Therapy on an Individual with Complex Communicative, Cognitive and Behavioural Needs Following Acquired Brain Injury}

Bronwyn Moorhouse, ${ }^{1,2}$ Erica Mainprize, ${ }^{2}$ Jacinta Douglas, ${ }^{3}$ and ${\text { Caroline } \text { Fisher }^{4}}^{4}$

${ }^{1}$ Speech Pathology Department, Austin Health, Kew, Australia

${ }^{2}$ Brain Disorders Program, Austin Health, Kew, Australia

${ }^{3}$ Living with Disability Research Centre, School of Allied Health, La Trobe University, Bundoora, Australia

${ }^{4}$ Allied Health - Psychology, Melbourne Health, Parkville, Australia

$\boldsymbol{B}$ ackground and aims: Individuals with severe communication and memory deficits coupled with psychiatric difficulties face enormous challenges engaging in everyday life. This study compared the participation levels of individuals with Acquired Brain Injury during a more traditional discussion group versus an art therapy group - where pressure to talk, listen and remember was much reduced.

Method: Data on participation levels across weekly group sessions was collected for nine individuals using an empirical single case time series ABA design. Six baseline data points and a further six return to baseline data points were collected for sports discussion groups and 10 intervention data points for art therapy groups. Speech Pathologist facilitated groups discussed recent sporting news. Art Therapist facilitated groups had different weekly artistic themes - e.g., mask making or stenciling. Participants were free to come and go at will. Interviews with facilitators after intervention and return to baseline sessions were also conducted. 
Results: Preliminary results focus on one participant who found attendance at 'talking' groups particularly challenging. He actively declined to attend any baseline discussion groups, but participated in nearly all art therapy groups. He then also attended most subsequent discussion groups. His participation is captured in a time series graph with interview data used to illustrate findings. Conclusions: Results provide preliminary evidence for art therapy supporting group participation in individuals challenged by 'talking' groups. Further individual and group study data will provide greater insights into who might benefit from this approach which also promotes non-verbal forms of individual expression.

\title{
Relationships in Aphasia Therapy: Why Do We Work as We Do?
}

\author{
Felicity Bright, ${ }^{1}$ Stacie Attrill, ${ }^{2}$ and Deborah Hersh $^{3}$ \\ ${ }^{1}$ Centre for Person Centred Research, Auckland University of Technology, Auckland, New Zealand \\ ${ }^{2}$ Department of Speech Pathology, Flinders University, Adelaide, Australia \\ ${ }^{3}$ Speech Pathology, School of Medical and Health Sciences, Edith Cowan University, Perth, Australia
}

$\boldsymbol{R}$ ackground and objectives: Relationships are central to speech pathology

$B$ practice, influencing rehabilitation processes and clients' experiences and outcomes. However, there has been limited exploration how and why therapists construct and enact relationships as they do. The objective of this research was to use sociological theories to examine client-practitioner relationships and, in particular, what influenced how relationships were enacted.

Method: A case study of one speech pathologist-client dyad. Data included observations of six interactions between them, two interviews with the client, and four interviews with the speech pathologist. Three sociological theories (critical theory, Social Interaction and Structuration Theory) informed data analysis.

Results: The therapeutic relationship occurred in a complex context. While it was considered important, relational work did not hold the same legitimacy as other aspects of practice. Multiple forms of power relations were evident, expressed subtly in interaction through word choices, proxemics, touch, use of time and space. While the therapist exerted power with the client, she herself was influenced by, multiple power relationships with other practitioners. This was evident in her seemingly subconscious desire to present herself in different ways to different stakeholders. Structural influences included the dominance of the medical model, and conceptualisations of legitimate practices. While at face value, the therapist had agency in her actions, analysis demonstrated this was constrained by internal and external structures.

Conclusion: Sociological theory can help reveal implicit assumptions about relationships and relational practices, and can open up different ways of critically reflecting on speech pathology practice. 
Session 12: Understanding and Meeting the Needs of Families

\title{
Positive Behaviour Support (PBS) Following a Brain Injury: The Development of a Family Education Workbook
}

\author{
Alinka Fisher, ${ }^{1}$ Michelle Bellon, ${ }^{1}$ Sharon Lawn, ${ }^{2}$ and Sheila Lennon ${ }^{3}$ \\ ${ }^{7}$ Disability and Community Inclusion, College of Nursing and Health Sciences, Flinders University, \\ Adelaide, Australia \\ ${ }^{2}$ Department of Psychiatry, Flinders University, Adelaide, Australia \\ ${ }^{3}$ Discipline of Physiotherapy, Flinders University, Adelaide, Australia
}

$\boldsymbol{R}$ ackground and aims: Family members often become the primary caregiver following brain injury and experience significant unmet information needs regarding behavioural changes. The level of information provided is often not understood or relevant to families' individual situations. This emphasises the need for accessible information provided to families in layperson language, which is relevant to individualised and community-based contexts.

Methods: The development of the Family Education Workbook was informed by a systematic review examining family involvement in behavioural interventions following brain injury and a Delphi study. Rehabilitation professional $(n=3)$ and family caregivers $(n=6)$ then reviewed the 82-page workbook and provided feedback regarding the accessibility and usefulness of the information presented.

Results: The Family Education Workbook consists of seven modules including information regarding common neuro-behavioural sequelae of brain injury, factors contributing to the occurrence of behaviours of concern, and principles of PBS. It is written with a strength-focus and family-centred approach, with narrative illustrations and activities to support families in applying knowledge to their individual situations.

Conclusions: This workbook provides an important resource for families, increasing their capability to effectively support behavioural changes following brain injury in community settings. This stand-alone resource is used to support the education component of a family-directed behaviour support program (FAB-PBS), which is currently being trialled in a feasibility study funded by the Lifetime Support Authority (LSA).

\section{Developing and Embedding a Family/Carer Support Group into an Inpatient Acquired Brain Injury Rehabilitation Service}

Anna Uliando, and Jacinta Sadler

Acquired Brain Injury Rehabilitation Centre, Alfred Health, Melbourne, Australia

$\boldsymbol{B}$ ackground and aims: The Alfred Health Acquired Brain Injury (ABI) B Rehabilitation Centre opened in 2014, comprising a state-wide inpatient and community-based rehabilitation service. The model of care recognises that caregivers are essential in providing support, and increasing satisfaction and engagement in rehabilitation. However, this role can be demanding, often with negative impacts on health, wellbeing and lifestyle. Research on family and carer wellbeing indicates positive evidence for interventions with education and psychosocial support. This paper aims to present on the development and implementation of a specialised group-based intervention for family/carers of inpatients with $\mathrm{ABI}$.

Method: Following review of the research literature, consumer feedback and collaboration between inpatient psychology and social work teams, a treatment programme was designed and implemented. Using an open-ended format, 
the programme includes four sessions designed to increase knowledge, share and normalise experiences, strengthen coping resources, and alleviate stress. Evaluations are undertaken following each session to assess the effectiveness of the group.

Results: Results from evaluations have been positive, whilst also identifying areas for improvement, and barriers for successful implementation. In response, the programme has been revised a number of times. A summary of the sessions and findings from evaluations will be presented.

Conclusions: The provision of a specialised group-based intervention for family/carers in the inpatient setting has been valued by participants, and embedded into everyday practice. This can be considered as a potentially useful format for services. Ongoing evaluation and further research is required to inform future groups in addressing the needs of families/carers of patients with ABI.

\title{
Learnings and Evaluations: Key Principles for Establishing Successful Peer Support for People Living with Acquired Brain Injury and Their Families
}

\author{
Jennifer Farnden, ${ }^{1}$ and Michelle Bellon ${ }^{2}$ \\ ${ }^{1}$ Jennifer Farnden (previously Families4Families), Australia \\ ${ }^{2}$ Flinders University, Australia
}

\begin{abstract}
$B$ ackground and aims: Families4Families (F4F) is a South Australian (SA) peer support organisation that has assisted people impacted by acquired brain injury (ABI) and their families to build resilience and maximise wellbeing and quality of life by providing information and education sessions, social opportunities, on-line resources and other family centric supports via peer-based programmes delivered across SA since 2012. This paper aims to outline key components in establishing a successful peer support model for people impacted by ABI and present evaluation findings.

Method: Subsequent to an earlier study exploring the support needs of individuals with $\mathrm{ABI}$ and their families, F4F has implemented a series of support groups and activities since 2012 and these groups are continuing and developing. Ongoing data collection and evaluation has been part of the programme development process.

Results: F4F now has more than 660 members with 20 Local Support Groups operating across the state, including many rural and remote groups. Evaluation findings show benefits to members as indicated by improved quality of life and wellbeing through social connection, shared support, access to information and referrals to other services/supports.

Conclusions: Peer support is an important form of service provision. Families4Families believes that every person with ABI should be supported in their journey towards a good life full of choice and control and that peer support is a valuable and important component of the journey post-ABI for people living with $\mathrm{ABI}$ and their families.
\end{abstract}

\section{The Hidden Impact of Assaults on Families: Preliminary Results}

Annerley Bates, ${ }^{1}$ Vivien Houston, ${ }^{2}$ Lyndel Bates, ${ }^{3}$ and David Belsham ${ }^{3}$

${ }^{1}$ Brain Injury Rehabilitation Service, Princess Alexandra Hospital, Brisbane, Australia

${ }^{2}$ Metro South Health, Queensland Health, Brisbane, Australia

Chool of Criminology and Criminal Justice and Griffith Criminology Institute, Griffith University, Brisbane, Australia 
Background: Health data suggests that a significant proportion (12\%) of individuals admitted to hospital with a traumatic brain injury (TBI) within Australia do so as the result of an assault (Helps, Henley \& Harrison, 2008; Morisse et al., 2014). The costs associated with these types of injuries are substantial and these injuries place a significant burden on informal caregivers, such as parents, spouses and siblings.

Objectives: The aim of this research is to explore the impacts on the informal caregivers who provide support to those who receive an assault related injury. Methods: Eight in-depth interviews were conducted by a Senior Social Worker with informal caregivers of individuals who have a TBI as the result of an assault or alleged assault at a state-wide Brain Injury Service. A thematic analysis utilising an interpretivist-constructivist approach was used to explore the preliminary themes arising from this research.

Results: The interviews indicate that these injuries have a significant impact on the informal caregivers. This includes personal impacts such as fatigue, personal safety and psychological wellbeing. Additionally, informal caregivers are concerned about future employment prospects for the injured person.

Conclusions: There is no research regarding how TBIs received as the result of an assault or alleged assault affect informal caregivers. This research addresses this gap. Thus, the research will be useful in developing a targeted intervention for this population in order to provide more effective support.

\title{
Understanding Family Workload and Capacity Following Catastrophic Injury: Preliminary Results
}

\author{
Carolyn Ehrlich, ${ }^{1}$ Annerley Bates, ${ }^{1,2}$ Grahame Simpson, ${ }^{1,4}$ Elizabeth McNevin, ${ }^{1,3}$ \\ Michelle McIntyre, ${ }^{1,5}$ and Michelle Foster ${ }^{1}$ \\ ${ }^{1}$ The Hopkins Centre, Griffith University, Brisbane Australia \\ ${ }^{2}$ Brain Injury Rehabilitation Service, Metro South Health, Queensland Health, Brisbane, Australia \\ ${ }^{3}$ Spinal Injury Unit, Princess Alexandra Hospital, Brisbane, Australia \\ ${ }^{4}$ Brain Injury Rehabilitation Research Group, Ingham Institute of Applied Medical Research \\ ${ }^{5}$ Synapse, Queensland
}

$O$

bjectives: Brain injury disrupts life narratives-people are biographically and geographically displaced, and existing social structures are thrown into disarray. This study aims to: (1) explore how injury impacts entire family networks; (2) conceptually move beyond the notion of care to explore the work associated with living after injury and (3) investigate how work is allocated and distributed among family networks.

Methods: This mixed methods study incorporates thematic and social network analysis of semi-structured interviews to understand how catastrophic injury impacts an entire family network.

Results: A preliminary analyses of in-depth interviews indicates that work is differentially distributed among family networks according to pre-existing life skills of injured persons and family members and according to individual and specialised knowledge about the injured person. The manner in which these pre-existing skills and knowledge interact with the work of healthcare professionals is variable and appears to be highly individualised. Resilient adaptation also seems to be connected to pre-existing skills and knowledge of members within the network in combination with decisions about, and capacity for, labour distribution amongst the network.

Conclusions: Conceptually, this research moves beyond injured individual and carer dyads to explore the impact of injury on entire family networks. It also moves beyond traditional notions of care by exploring the relationships 
between work, workload distribution and entire family network capacity in the context of brain injury.

\title{
Session 13: Sensory and Motor Considerations in Rehabilitation
}

\section{New Findings in Olfactory Impairment Following Traumatic Brain Injury}

Melanie Drummond, ${ }^{1,2,3}$ Jacinta Douglas, ${ }^{1,4,5}$ and John Olver ${ }^{2,3}$

${ }^{1}$ College of Science, Health and Engineering, La Trobe University, Victoria, Australia

${ }^{2}$ Epworth Monash Rehabilitation Medicine Unit, Melbourne Australia

${ }^{3}$ Epworth Rehabilitation, Epworth HealthCare, Melbourne, Australia

${ }^{4}$ NHMRC Clinical Centre of Research Excellence in Brain Recovery, Melbourne, Australia

${ }^{5}$ Summer Foundation, Melbourne, Australia

\begin{abstract}
$B$ ackground and objectives: Olfactory Impairment (OI) is a consequence of traumatic brain injury (TBI). It's an enduring impairment which significantly impacts on everyday activities, regardless of its severity.

This presentation shares the findings of a longitudinal study which investigated the incidence, recovery and predictive factors of outcome relating to OI following TBI. The lived experience of OI was also explored from the perspective of those who continued to deal with the everyday impact of OI over a 12-month period.

Methods: Participants $(n=134)$ were recruited via a consecutive admission series following admission to the Acquired Brain Injury (ABI) Unit at Epworth Rehabilitation. The presence of OI was assessed using the University of Pennsylvania Smell Identification Test (UPSIT). Individuals assessed as having OI at each time point were invited to participate in follow-up assessment. Open ended interviews were conducted at both follow-up time points.

Results: $89(66.42 \%)$ of the participants were assessed as having OI on initial assessment. At 6 months $74 \%$ of the participants $(n=47)$ had persisting OI and at 12 months this increased to $86 \%(n=35)$. Age at injury, duration of PTA and facial fractures were shown to make no significant contribution to prediction of performance over time, with performance on initial UPSIT accounting for the most variability.

Conclusions: OI post TBI is enduring and can have significant consequences for the individuals who experience it. It is imperative that clinicians working with rehabilitation understand the widespread implications and ensure the individuals they work with receive assessment and comprehensive management.
\end{abstract}

\section{Variability in Visual Dysfunction Following Severe Traumatic Brain Injury (TBI): Consideration of Compensatory Approaches}

\author{
Suzane Vassallo, ${ }^{1}$ and Jacinta Douglas ${ }^{1,2}$ \\ ${ }^{1}$ School of Allied Health, College of Science, Health \& Engineering, La Trobe University, Melbourne, \\ Australia \\ ${ }^{2}$ Summer Foundation, Melbourne, Australia
}

ackground and objectives: A range of visual deficits have been described
following traumatic brain injury (TBI), with almost $50 \%$ of the current
literature reporting these deficits via single case studies. This study aimed to
undertake a visual assessment within a group of participants with severe TBI to:
(i) describe the nature of visual deficits recorded and (ii) identify compensatory
approaches that patients present with to improve functional visual outcomes. 
Method: Fifteen participants (14 male) participated in this investigation, which formed part of a larger study. Participants were aged 21-60 years (mean + SD: $37.5 \pm 10.8$ years) and sustained a severe TBI between 1 and 20 years ago $(7.7+6.1$ years). Various orthoptic assessments were performed.

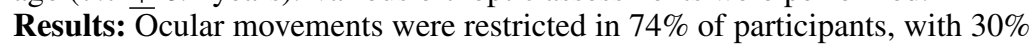
being bilaterally affected. Saccades were impaired in $70 \%$ (7/10 participants assessed), which included reduced velocity (20\%), inaccuracy (40\%) and increased latency $(40 \%)$. Eighty percent $(12 / 15)$ presented with strabismus which was either horizontal $(67 \%)$ or vertical $(40 \%)$. Only three participants $(20 \%)$ reported double vision on the day of testing. However, nine participants $(60 \%)$ had either adopted or developed compensatory functions to assist in maintaining single vision, including: eye closure/covering, anomalous head posture and alternate fixation.

Conclusions: Not all TBI patients present with the same visual deficits and, when they do, it is not to the same extent. Many participants adopted compensatory methods to obtain single vision. A longitudinal investigation is required to determine the variation in visual deficits following TBI.

\title{
Barriers and Enablers to the Implementation of Sustainable Publicly Funded Constraint Induced Movement Therapy (CIMT) Programs: The ACTIveARM Project
}

\author{
Lauren Christie, ${ }^{1,2,3}$ Meryl Lovarini, ${ }^{1}$ Annie McCluskey, ${ }^{1}$ Abigail Hunter, ${ }^{2,3}$ and \\ Reem Shuhaiber ${ }^{2,3}$ \\ ${ }^{1}$ The University of Sydney, Sydney, Australia \\ ${ }^{2}$ Brain Injury Rehabilitation Research Group, The Ingham Institute of Applied Medical Research, \\ Liverpool, Australia \\ ${ }^{3}$ Liverpool Brain Injury Rehabilitation Unit, Liverpool, Australia
}

$\boldsymbol{R}$ ackground: Constraint Induced Movement Therapy (CIMT) is an effective intervention for arm recovery following stroke and traumatic brain injury. Despite strong evidence for CIMT, there is a knowledge translation gap between research and practice.

Aim: To identify barriers and enablers to CIMT implementation and sustainability across multiple health services in South Western Sydney Local Health District (SWSLHD), Australia.

Method: Qualitative design using grounded theory methods. Semi structured focus group interviews were conducted with nine neurorehabilitation therapy teams across SWSLHD. One to one interviews were also conducted with two allied health managers. Interviews were audio recorded, transcribed and imported into Nvivo for analysis. The Theoretical Domains Framework (TDF) was used to inform the structure of interview questions, guide data analysis and generate key themes.

Results: Thirteen interviews were conducted with occupational therapists, physiotherapists, allied health assistants and managers. Barriers to CIMT implementation included (1) Lack of therapists' knowledge of the CIMT protocol, (2) Therapists' lack of confidence in their ability to deliver CIMT and (3) Concerns regarding inadequate resources to deliver CIMT. Enablers included (1) Therapists' belief that the intervention was part of their professional role and (2) Concerns about the potential negative impact of not delivering the intervention. Therapists were optimistic that they would be able to use their existing skills to deliver the intervention once further training had been provided.

Conclusion: The results of this study will inform the development of an implementation package to support therapy teams in overcoming an 
evidence-practice gap; and accelerate arm recovery through routine delivery of an effective intervention.

\section{Implementation of Sustainable Publicly Funded Constraint Induced Movement Therapy (CIMT): The ACTIveARM Project}

Lauren Christie, ${ }^{1,2,3}$ Meryl Lovarini, ${ }^{1}$ Annie McCluskey, ${ }^{1}$ Reem Shuhaiber, ${ }^{2,3}$ and Nicola Acworth ${ }^{2}$

${ }^{1}$ The University of Sydney, Sydney, Australia

${ }^{2}$ Brain Injury Rehabilitation Research Group, The Ingham Institute of Applied Medical Research, Liverpool, Australia

${ }^{3}$ Liverpool Brain Injury Rehabilitation Unit, Liverpool, Australia

ntroduction: Constraint Induced Movement Therapy (CIMT) is an effective intervention for arm recovery following stroke and traumatic brain injury. Despite the recommended use of CIMT in clinical guidelines, there is a knowledge translation gap between research and practice.

Objectives: To establish if a 2-week publicly funded CIMT programme can be translated into practice and sustained over two years across multiple health services in South Western Sydney Local Health District (SWSLHD), Australia. Method: Before and after study design involving nine therapy teams across SWSLHD.

Teams received a CIMT Implementation Package including; (a) a 2 day training workshop, (b) regular audit feedback about their practice measured at baseline and 3 monthly intervals post training, (c) poster reminders and (d) support and mentoring from a community of practice about CIMT delivery.

Primary Outcome: The proportion of eligible patient participants that were offered and received CIMT.

Results: Baseline file audits $(n=180)$ indicated that $38 \%$ of patients were eligible for CIMT during rehabilitation, however, less than $2 \%$ of patients were offered and provided with CIMT. Baseline audit data suggested CIMT was not delivered with fidelity. Preliminary repeat file audit results completed 3 months post CIMT training workshop $(n=148)$ indicated an improvement in CIMT screening and delivery, with $51 \%$ of eligible patients $(n=49)$ offered CIMT and $27 \%(n=13)$ receiving a programme. Sustainability of these changes in practice over 15 months will be explored.

Conclusion: Therapy teams will overcome an evidence-practice gap through routine delivery of a highly effective intervention. 


\title{
Early Target Selection and Evidence Accumulation is Impaired in the Left Hemifield After Right Hemisphere Stroke
}

Megan H. O'Neill, ${ }^{1}$ Gerard M. Loughnana, ${ }^{2,3}$ Daniel P. Newman, ${ }^{1}$ Méadhbh B. Brosnan, ${ }^{1,3,4}$ Renerus J. Stolwyk, ${ }^{1}$ Peter W. New, ${ }^{5,6,7}$ Trevor T-J. Chong, ${ }^{1}$ Redmond G. O'Connell, ${ }^{3,4}$ and Mark A. Bellgrove ${ }^{1,3,4}$

${ }^{7}$ Monash Institute for Cognitive and Clinical Neurosciences (MICCN), Monash University, Melbourne, Australia

${ }^{2}$ School of Engineering, Trinity College Dublin, Dublin 2, Ireland

${ }^{3}$ Trinity College Institute of Neuroscience, Trinity College Dublin, Dublin 2, Ireland

${ }^{4}$ School of Psychology, Trinity College Dublin, Dublin 2, Ireland

${ }^{5}$ Department of Rehabilitation and Aged Care, Medical Program, Monash Health, Victoria, Australia ${ }^{6}$ Epworth-Monash Rehabilitation Medicine Unit, Southern Medical School, Monash University, Victoria, Australia

${ }^{7}$ Department of Epidemiology and Preventive Medicine, Faculty of Medicine, Nursing and Health Sciences, Monash University, Victoria, Australia

$\boldsymbol{R}$ ackground and objectives: Hemispatial neglect is a disabling neurologB ical syndrome defined as the inability to detect, respond to and orient towards stimuli contralateral to cerebral damage. It is more common and severe following right hemisphere stroke, resulting in left inattention. Despite decades of investigation, little is known about the underlying neurophysiological processes.

Method: A perceptual decision-making paradigm and electroencephalography were used to isolate and measure reaction time $(\mathrm{RT})$, the $\mathrm{N} 2_{(\mathrm{c})} / \mathrm{N} 2_{(\mathrm{i})}$, eventrelated potentials known to index attention orienting signals, and the CPP, an evidence accumulation signal. The sample included five right hemisphere stroke patients with recovered neglect (3 neglect and 2 severe neglect), 2 right hemisphere stroke participants without neglect and 27 healthy control participants.

Results: Behavioural results confirmed neglect in stroke patients, with significantly slower RTs for left hemifield targets $(p<.05)$. In neglect participants, single trial analysis showed evidence of dysfunctional attention orienting for left hemifield targets (right hemisphere $\mathrm{N} 2_{(\mathrm{c})}$ ) but compensation from the left hemisphere in the form of the $\mathrm{N} 2_{(\mathrm{i})}$. Importantly, this compensation was not evident in participants with severe neglect. Evidence accumulation, as measured by the CPP, was important for sound behavioural performance in neurologically healthy participants, neglect participants and non-neglect participants, with greater CPP slope related to faster RTs in both hemifields.

Conclusion: This work confirms that a perceptual decision-making framework can have utility in understanding the neurophysiological basis of spatial attention deficits. With further work, this approach could be translated to the clinical setting to improve assessment and better track neglect recovery over time.

\section{Peripheral Auditory Dysfunction Secondary to Traumatic Brain Injury: A Systematic Review of Literature}

\author{
Bojana Šarkić, ${ }^{1}$ Jacinta. M. Douglas, ${ }^{1,2}$ and Andrea Simpson ${ }^{1}$ \\ ${ }^{1}$ Discipline of Audiology, School of Allied Health, College of Science, Health and Engineering, La \\ Trobe University, Bundoora, Victoria 3083, Australia \\ ${ }^{2}$ Summer Foundation, Victoria, Australia
}

$\boldsymbol{R}$ ackground and aims: Despite numerous studies demonstrating hearing $B$ loss post Traumatic Brain Injury (TBI) there has been no systematic 
investigation of the prevalence, nature and severity of peripheral hearing loss. To understand the effects of non-blast related TBI on peripheral auditory function in adults, as measured through basic and advanced audiological assessments.

Method: An English-language systematic search using MEDLINE, CINAHL, PsychInfo, Pubmed and hand-searching of reference lists was conducted from January 1st 1990 to October 31st 2016. After independent review by the authors, 20 of 281 originally identified articles were retained. Audiological findings were extracted and synthesised across studies.

Results: Using the Oxford Centre for Evidence Based Medicine levels of evidence (2009), 3b was the highest level of evidence within the review. Sensorineural hearing loss was the most consistent auditory deficit reported post TBI.

Conclusion: The range and frequency of auditory dysfunction in TBI patients remain unclear. Future research should focus on understanding the nature, frequency and change of auditory deficits over time following TBI. Knowledge in this area will provide crucial information for clinicians and facilitate the development of diagnostic and best practice guidelines which currently are lacking for the management of this patient population.

\section{Session 14: Working with Electronic Services and Delivery}

\section{A Systematic Review of the Efficacy of Telerehabilitation for Adults with Traumatic Brain Injury}

Tamara Ownsworth, Urska Arnautovska, Elizabeth Beadle, David H. K. Shum, and Wendy Moyle

Menzies Health Institute Queensland, Griffith University, Brisbane, Australia

$\boldsymbol{B}$ ackground and aims: Technology is increasingly being used to provide 3 rehabilitation remotely to people with neurological disorders. Telerehabilitation platforms have the potential to expand and decentralise service delivery and reduce global health disparities in accessing rehabilitation. This review aimed to identify and appraise studies evaluating the efficacy of telerehabilitation for adults with traumatic brain injury (TBI).

Methods: A systematic search of four databases (Cochrane Library, MEDLINE, CINAHL \& PsycINFO) was conducted from January 1980 to April 23 2017 for studies evaluating the efficacy of telerehabilitation for adults with TBI. Articles were independently assessed by two reviewers for eligibility and rated on methodological quality using 16 criteria related to internal validity, descriptive and statistical characteristics.

Results: 13 eligible studies, including 10 randomised controlled trials (RCTs) and three pre-post group studies $(n \geq 10)$ were identified. These evaluated the feasibility and/or efficacy of telephone-based (10 studies) and Internet-based (three studies) interventions. Overall, the evidence of efficacy was somewhat mixed. The most common study design evaluated the efficacy of telephonebased interventions relative to usual care, for which 4 out of 5 RCTs reported positive effects at post-intervention $(d=.28-.51)$. The feasibility of Internetbased interventions was mostly supported; however, the efficacy could not be determined due to insufficient studies.

Conclusions: Structured telephone interventions appear to be effective for improving particular outcomes following TBI. Further research on controlled 
studies of Internet-based therapy and comparisons of the clinical and costeffectiveness of in-person and telerehabilitation formats is needed.

\title{
Telehealth Delivery of Memory Rehabilitation Following Stroke
}

\author{
David Lawson, ${ }^{1,2}$ Rene Stolwyk, ${ }^{1,2}$ Jennie Ponsford, ${ }^{1,2}$ and Dana Wong ${ }^{1,2}$ \\ ${ }^{1}$ School of Psychological Sciences \& Monash Institute of Cognitive \& Clinical Neurosciences, Monash \\ University, Melbourne, Australia; \\ ${ }^{2}$ Monash-Epworth Rehabilitation Research Centre, Melbourne, Australia
}

\begin{abstract}
$B$ ackground and aims: Memory difficulties are reported by around half of stroke survivors, and impact independence and work capacity. Group rehabilitation programmes improve everyday memory function, however, many encounter barriers to participation in these programmes, due to mobility issues and geographic location. Telehealth delivery of memory rehabilitation could potentially overcome barriers; however, evidence is lacking that these delivery modes are feasible or result in similar positive outcomes.

Method: Participants with stroke $(N=31)$ completed a six-week memory skills training programme, allocated into group format, individual face-toface, or individual telehealth delivery using internet-based videoconferencing. Achievement of personalised, specific goals was monitored pre- and postintervention and six-week follow-up, using Goal Attainment Scaling as the primary outcome measure. Utilisation of compensatory memory strategies was measured using a self-report Strategy Checklist.

Results: $83.3 \%$ of memory group participants achieved at least one personal goal, while $100 \%$ of individual face-to-face and telehealth participants achieved at least one goal. Significant increase in strategy use was observed across all groups, with no significant difference between delivery modes.

Conclusions: The results suggest that individual and telehealth delivery of memory skills training are at least equivalent in effectiveness to group-based memory rehabilitation. These findings have significant implications for improving access to memory rehabilitation for stroke survivors across Australia.
\end{abstract}

\section{The Transition from Hospital to Home; Interim Findings from a Longitudinal Study of Indigenous Traumatic Brain Injury (TBI) in Northern Australia}

India Bohanna, ${ }^{1}$ Michelle Fitts, ${ }^{1}$ Jennifer Fleming, ${ }^{2}$ John Gilroy, ${ }^{3}$ Paul Maruff, ${ }^{4,5}$ Adrian Esterman, ${ }^{1,6}$ Katrina Bird, ${ }^{1}$ Taeha Condon, ${ }^{1}$ and Alan Clough ${ }^{1}$

${ }^{1}$ College of Public Health, Medical \& Veterinary Sciences, Division of Tropical Health and Medicine, James Cook University

${ }^{2}$ School of Health and Rehabilitation Sciences, The University of Queensland

${ }^{3}$ Faculty of Health Sciences, Centre for Disability Research and Policy, The University of Sydney

${ }^{4}$ CogState Ltd, Melbourne, Victoria

${ }^{5}$ Florey Institute of Neuroscience and Mental Health

${ }^{6}$ Sansom Institute for Health Research and School of Nursing and Midwifery, University of South Australia

$\boldsymbol{R}$ ackground and aims: Traumatic brain injury (TBI) is a leading cause of disability in Australia. Until now, Australian studies on outcomes after TBI have reported almost exclusively on metropolitan dwelling, mainstream (non-Indigenous) populations. This multi-site study aims to understand the 6-month transition period following TBI for Indigenous Australians in north Queensland and the Northern Territory. This abstract reports the overall interim findings from this ongoing study. 
Method: Indigenous Australians aged 18-65 were recruited from three hospitals following admission for TBI. A range of qualitative and quantitative data was collected examining previous head injuries, key sentinel events following injury, cognitive, mental health \& social outcomes at three time points during the 6 months following injury.

Results: Indigenous Australians experience a range of positive and negative outcomes following TBI, some of which are consistent with the literature in the mainstream population (e.g., financial strain, relationship breakdown). However, Indigenous Australians also report unique transition experiences (e.g., contact with the criminal justice system, self-discharge from hospital). Cognitive, mental health and previous injury data will also be reported.

Conclusions: The unique experiences and outcomes for Indigenous Australians following TBI suggest complex pre- and peri-injury factors that must be taken into account to ensure that rehabilitation and transition services are culturally and socially appropriate and effective for Indigenous Australians. Implication for the National Disability Insurance Scheme will also be discussed.

\section{Incidence of Emergency Department Presentations for Head Injury and Traumatic Brain Injury (TBI) in Adults in North Queensland, Australia}

India Bohanna, ${ }^{1}$ Adrian Esterman, ${ }^{1,2}$ Fintan Thompson, ${ }^{1}$ Michelle Fitts, ${ }^{1}$ Jennifer Fleming, ${ }^{3}$ John Gilroy, ${ }^{4}$ Paul Maruff, ${ }^{5,6}$ Katrina Bird, ${ }^{1}$ Taeha Condon, ${ }^{1}$ and Alan Clough ${ }^{1}$

${ }^{1}$ College of Public Health, Medical \& Veterinary Sciences, Division of Tropical Health and Medicine, James Cook University

${ }^{2}$ Sansom Institute for Health Research and School of Nursing and Midwifery, University of South Australia

${ }^{3}$ School of Health and Rehabilitation Sciences, The University of Queensland

${ }^{4}$ Faculty of Health Sciences, Centre for Disability Research and Policy, The University of Sydney

${ }^{5}$ CogState Ltd, Melbourne, Victoria

${ }^{6}$ Florey Institute of Neuroscience and Mental Health

$\boldsymbol{B}$ ackground and aims: To describe the incidence of Emergency Department presentations for Head Injury and Traumatic Brain Injury (TBI) in adults aged 15-64 over a 9-year period in North Queensland, Australia.

Method: Retrospective analysis of data from North Queensland Emergency Departments between 2007 and 2015 matched to Australian Bureau of Statistics population data for North Queensland. Main outcome measures: Incidence rate ratios for head injury and TBI presentations by Indigenous status, age, sex, year of presentation, remoteness, and socio-economic disadvantage.

Results: For both head injuries and TBIs, Indigenous people were more likely to present than Non-Indigenous people, males more likely to present than females, and presentation rates declined with age. Incidence increased with year of presentation only in the Indigenous population. Remote areas had a higher rate of presentations than urban areas, but this difference was primarily due to the non-Indigenous population. There was a tendency for more socioeconomically deprived areas to have higher rates of presentations, but again this was mainly seen in the non-Indigenous population.

Conclusions: The recent increase in presentations for head injury and TBI for the Indigenous population are of concern. Implications of the results for service delivery and the National Disability Insurance Scheme will be discussed. 


\section{Sentinel Events During Hospital Admission for Indigenous People Following Traumatic Brain Injury}

Michelle Fitts, ${ }^{1}$ Jennifer Fleming, ${ }^{2}$ Katrina Bird, ${ }^{1}$ Taeha Condon, ${ }^{1}$ John Gilroy, ${ }^{3}$ Alan Clough, ${ }^{1}$ Paul Maruff, ${ }^{4,5}$ Adrian Esterman, ${ }^{1,6}$ and India Bohanna ${ }^{1}$

${ }^{1}$ College of Public Health, Medical \& Veterinary Sciences, Division of Tropical Health and Medicine, James Cook University

${ }^{2}$ School of Health and Rehabilitation Sciences, The University of Queensland

${ }^{3}$ Faculty of Health Sciences, Centre for Disability Research and Policy, The University of Sydney

${ }^{4}$ CogState Ltd, Melbourne, Victoria

${ }^{5}$ Florey Institute of Neuroscience and Mental Health

${ }^{6}$ Sansom Institute for Health Research and School of Nursing and Midwifery, University of South Australia

$\boldsymbol{R}$ ackground and aims: Few studies have examined Indigenous Australian's experiences following hospital admission for Traumatic Brain Injury (TBI). This study examined key sentinel events (events, positive or negative, which arise during the transition period and may influence health and/or wellbeing) for Indigenous Australians during the period of hospital care following a TBI in three hospitals in northern Australia.

Method: Seventeen Indigenous patients (aged 18-65 years) were recruited within one week of discharge. The Sentinel Events Questionnaire \& associated qualitative interview was used to record events influencing the participant's transition experience.

Results: The most reported negative sentinel event was financial strain, primarily related to participants' inability to work or the termination of welfare benefits due to an extended period of hospital care. Relationship breakdown was frequently reported, with the events surrounding the TBI incident often a contributing factor to the breakdown. Contact with police was common when Police responded to the initial injury-related incident or where the incident may have required investigation. Almost all participants had accessed therapy or rehabilitation services. However, some participants desired additional services unrelated to their injury (e.g., alcohol counselling).

Conclusions: Indigenous patients experience a range of sentinel events during their stay in hospital. This data suggest key intervention points to enhance the transition from hospital to home for Indigenous Australians. Hospital and discharge services should consider the occurrence of these events with admitted Indigenous TBI patients and assist where possible. Addressing these events before discharge is likely to facilitate successful transitions back to the community. 


\title{
Challenges in Recruiting Indigenous Patients in a Multi-Site, Multi-State Study of the Transition from Hospital to Home Following Traumatic Brain Injury
}

\author{
Michelle Fitts, ${ }^{1}$ Taeha Condon, ${ }^{1}$ John Gilroy, ${ }^{2}$ Katrina Bird, ${ }^{1}$ Alan Clough, ${ }^{1}$ Jennifer \\ Fleming, ${ }^{3}$ Paul Maruff, ${ }^{4,5}$ Adrian Esterman, ${ }^{1,6}$ and India Bohanna ${ }^{1}$ \\ ${ }^{1}$ College of Public Health, Medical \& Veterinary Sciences, Division of Tropical Health and Medicine, \\ James Cook University \\ ${ }^{2}$ Faculty of Health Sciences, Centre for Disability Research and Policy, The University of Sydney \\ ${ }^{3}$ School of Health and Rehabilitation Sciences, The University of Queensland \\ ${ }^{4}$ CogState Ltd, Melbourne, Victoria \\ ${ }^{5}$ Florey Institute of Neuroscience and Mental Health \\ ${ }^{6}$ Sansom Institute for Health Research and School of Nursing and Midwifery, University of South \\ Australia
}

$\mathcal{R}$ ackground and aims: There is limited understanding of the challenges in conducting traumatic brain injury (TBI) research with Indigenous Australians. In this multi-site, multi-state project examining outcomes after TBI for Indigenous Australians, patient experiences around the time of injury and in hospital created ongoing recruitment and retention challenges for the research team. This presentation reports on the research team's experiences.

Method: Participant recruitment was conducted in two hospitals in the Northern Territory and Queensland, aiming to recruit Indigenous Australians (aged 18-65) following admission with TBI. Qualitative data collected during research staff's contact with patients, their family members and hospital staff in addition to team meetings and researcher reflections were analysed to identify challenges.

Results: Eligible patients discharging against medical advice, 'absconding' or being deemed by allied health staff as not having capacity to consent were the major recruitment barriers. Strict eligibility criteria including a minimum 24hour admission and emergence from post-traumatic amnesia (PTA) based on the Westmead PTA scale also hampered access to otherwise eligible patients. The research team often had to compete for patient access alongside treatment and discharge services. Broadening the participant eligibility criteria, building strong relationships with key hospital staff and adding additional hospitals for recruitment enabled the research team to overcome some of these challenges. Conclusions: Hospital-related recruitment is time- and resource-intensive, with several recruitment barriers in this setting. Project flexibility is required to ensure appropriate amendments can be made. Further commentary on implementing Indigenous research in hospitals will support researchers to develop appropriate recruitment methods. 
Session 15: Connecting Services: Criminal Justice, Health and Disability

\section{Translating the National Disability Insurance Scheme (NDIS) into Practice: Examining Choice, Collaboration and Capacity in the Specialist Rehabilitation Setting}

Vivien Houston, ${ }^{1}$ Amy Seymour-Jones, ${ }^{2}$ Meghan Nolan, ${ }^{3}$ Michele Foster, ${ }^{4}$ and Pim Kuipers $^{5}$

${ }^{7}$ Metro South Health, Queensland Health, Brisbane, Qld, Australia

${ }^{2}$ Brain Injury Rehabilitation Service, Princess Alexandra Hospital, Brisbane, Qld, Australia

${ }^{3}$ Spinal Injuries, Princess Alexandra Hospital, Brisbane, Qld, Australia

${ }^{4}$ The Hopkins Centre, Brisbane, Qld, Australia

${ }^{5}$ Centre for Functioning and Health Research, Brisbane, Qld, Australia

$\boldsymbol{R}$ ackground: Translation and implementation of policy innovations like 3 the National Disability Insurance Scheme (NDIS) are often uncertain in practice and tensions typically arise for the agents and other stakeholders of implementation. How services separate 'disability-related' needs from those associated with mainstream services, such as health, is as yet unresolved. This lack of clarity makes it difficult to conceptualise the boundaries between different services in practice, hindering choice and collaboration (core aspirations of the reform) and negatively impacting consumer experience.

Objectives: This study will provide an understanding of: (1) how frontline health services are responding to implementation of the NDIS and associated challenges; (2) how concepts of choice and collaborative support planning are being operationalised at the health-disability interface and (3) the emergency resources and capacity issues related to the practice of choice based and collaborative decision making.

Methods: Process mapping, shaped around the NDIS pathway and concepts of choice, collaboration and capacity, will be used to illustrate the operation of the NDIS in practice and the response from the health service.

Results: The preliminary results will be presented.

Conclusions: This project is at the forefront of the NDIS implementation process in Queensland and will contribute to an understanding of the NDIS in practice at the health-disability interface. More specifically, it identifies key capacity and collaboration issues, including the resources required at the practice and system level to facilitate choice and collaborative decision making for patients and families.

\section{Connecting the Health System and the National Disability Insurance Scheme (NDIS) to Create Positive Outcomes for People with Complex Disability}

Cathy Bucolo, and Joanna Stewart

Summer Foundation, Australia

$\boldsymbol{R}$ ackground and aims: The range of health and disability supports people with complex disability require, along with the new language and concepts of the National Disability Insurance Scheme (NDIS), have caused many health practitioners to think, it may never be possible to connect the two worlds of health and the NDIS for the people they support. This project aimed to support younger people with complex needs in the health system to facilitate their timely discharge into the community to live 'an ordinary life' and avoid entry into residential aged care (RAC).

Method: The Summer Foundation was funded by the National Disability Insurance Agency (NDIA) to work closely with four Local Health Networks 
(LHNs) across Australia, co-designing resources for people with a disability and health practitioners that improve NDIS access and planning.

Results: A number of barriers and issues were identified for younger people with complex disability when they are faced with transition between systems in Health and the NDIS. Practical strategies and resources were developed to guide change in day to day practice to offset barriers and facilitate the connection between the health system and the NDIS. These resources have been made available to all LHNs across Australia

Conclusions: Health professionals will need to adapt their practice in order to meet the demands of changes within the health and disability sectors.

\title{
'My Home Space': An Interactive, Online Tool to Explore Specialist Disability Accommodation in the National Disability Insurance Scheme (NDIS)
}

\author{
Libby Callaway, ${ }^{1,2}$ and Kate Tregloan ${ }^{3}$ \\ ${ }^{1}$ Occupational Therapy Dept, Monash University, Frankston, Australia \\ ${ }^{2}$ Neuroskills Pty Ltd, Sandringham, Australia \\ ${ }^{3}$ Architecture Dept, Monash University, Caulfield, Australia
}

\begin{abstract}
$B$ ackground and objectives: Australia's NDIS changes the way support is funded for people with acquired brain injury (ABI). This includes separation of support for independent living from housing 'bricks and mortar'. The new Specialist Disability Accommodation (SDA) policies and funding offer opportunity for people with ABI experiencing high daily support needs to consider a range of SDA housing. However, SDA guidelines may be hard to understand and implement.

Method: Using interdisciplinary research, this project: (1) investigated exemplar housing sites nationally that align with the NDIS SDA framework; (2) explored housing, technology and support design from the perspective of tenants (using published measures and qualitative methods); (3) held a housing roundtable with Government and National Disability Insurance Agency representatives and (4) converted SDA guidelines, roundtable and research findings into an interactive information tool, called 'My Home Space'. This tool was tested with NDIS participants, families, architects, occupational therapists, access consultants and government, then finalised.

Results: 'My Home Space' is a freely available on-line tool that allows the user to filter SDA design categories and housing types, enter 3D 'virtual homes', and examine features for housing build or retrofit. Information can be filtered by activity type and information source, using interactive hotspots and 2-D detail drawings. It also produces printable reports on 16 SDA combinations. Conclusions: 'My Home Space' is the first digital tool that integrates government policy with tenant experience. It offers accessible information for people with disability and families, as well stakeholders, working to achieve housing outcomes in the NDIS.
\end{abstract}

\section{"It was worse than being in jail": The Experience of Living in Residential Aged Care for Young People with Acquired Neurological Conditions}

\author{
Lucy Knox, ${ }^{1,2}$ Sandra Barry, ${ }^{1,2}$ and Jacinta Douglas ${ }^{1,2}$ \\ ${ }^{1}$ Living with Disability Research Centre, La Trobe University \\ ${ }^{2}$ Summer Foundation, Victoria
}

Background and aims: Over 6000 people under the age of 65 currently live
in residential aged care (RAC) in Australia. Although previous research
has measured the negative consequences associated with life in RAC for young 
people, there has been less attention on their lived experience. Subsequent to our previous research into the experience of entry to RAC, the aim of this study was to understand the experience of living in RAC for young people with $\mathrm{ABI}$ and their families.

Method: A qualitative research design underpinned by a constructivist paradigm and hermeneutic approach was adopted. Data included 64 written and verbal submissions from young people and their family members to the 2015 Senate Inquiry into the Adequacy of existing residential care arrangements available for young people with severe physical, mental or intellectual disabilities in Australia. Data was analysed using thematic analysis.

Results: Two central themes emerged from analysis of the rich insights provided by respondents. The first theme revealed life in RAC as a form of incarceration for young people with ABI. Their experience of imprisonment was reflected in four subthemes: (1) Being isolated, (2) Experiencing a failure of caring, (3) Having my potential restricted and (4) Not having a home. The second theme captured the impact of this imprisonment in shaping selfconcept.

Conclusions: The findings of this research highlight the significant restriction of rights experienced by young people living in RAC and the critical role that professionals can play in response. Several recommendations for policy and practice will be presented.

\section{Session 16 - How to Session 4}

\section{Cultural Understanding and Assessment of Neurocognitive Disability Amongst Marginalised Aboriginal and Torres Strait Islander People}

Clare Townsend, ${ }^{1,3,4}$ Jennifer Cullen, ${ }^{1,2}$ Paul White, ${ }^{2}$ and Kim McCoomb ${ }^{5}$

${ }^{1}$ Synapse, Brisbane, Australia

${ }^{2}$ Department of Communities (Disability Services), Specialist Disability Services Assessment \& Outreach Team, Brisbane, Queensland, Australia

${ }^{3}$ Griffith University Logan, Queensland, Australia

${ }^{4}$ James Cook University, Cairns, Queensland, Australia

${ }^{5}$ Anglicare North Queensland, Cairns, Queensland, Australia

C ynopsis: Limited research exists about Neurocognitive Disability (NCD) $\mathcal{N}$ in Aboriginal and Torres Strait Islander populations. There is a lack of culturally safe assessment tools. Sub-optimal service responses result in overrepresentation in hospitals, mental health services, prisons and criminal justice settings. The authors, Traditional Owners, Elders, and communities have worked to develop the Guddi Protocol, a culturally safe screen to establish accurate NCD prevalence and qualitative data amongst Aboriginal and Torres Strait Islander peoples. The Guddi Protocol enables Aboriginal and Torres Strait Islander people to engage with the assessment process and facilitates understanding of respondents' experience of disability and functionality. Research using the Guddi Protocol finds high levels of complex disablement amongst Aboriginal and Torres Strait Islander people, including NCD, psychosis and depression, PTSD and untreated illness.

The Guddi can be utilised in a range of contexts by culturally competent people and can assist with NDIS eligibility processes. This mini-masterclass will introduce participants to NCD amongst Aboriginal and Torres Strait Islander people, challenges to establishing their experience of NCDs and the Guddi Protocol, a culturally safe screening and assessment tool. Quantitative 
and qualitative research and a case example of the application of the Guddi will be presented. Participants will review the Protocol and engage in discussion as to its applicability to practice, in human services within a NDIS context.

Participants will have greater understanding of the nature of NCD amongst marginalised people, experience of a culturally safe assessment tool and opportunity to reflect on the usefulness of this tool to practice.

Level aimed for: Intermediate

Learning objectives: A delegate attending this session can expect to have:

- Greater understanding of the nature of NCD amongst marginalised people,

- Greater understanding of cultural safety when engaging with Aboriginal and/or Torres Strait Islander peoples;

- Experience of a culturally safe assessment tool;

- Participants are able to discuss and consider the applicability of the tool to practice.

\title{
Session 17 - How to Session 5
}

\section{Implementing and Facilitating an Effective Memory Skills Group}

\author{
Dana Wong, ${ }^{1}$ and Sandy Grayson ${ }^{2}$ \\ ${ }^{7}$ Monash Psychology Centre, School of Psychological Sciences, Monash University \\ ${ }^{2}$ Community Rehabilitation, Monash Health
}

ynopsis of session: Group-based memory skills programmes can be an $\mathcal{O}$ effective and cost-efficient component of rehabilitation following acquired brain injuries and illnesses, enabling participants to develop habits and strategies that optimise their daily functioning. The Monash Memory Skills Group, which is based on the Making the Most of Your Memory programme (ASSBI Resources), has been running since 2014 and includes psychoeducation, training in internal and external memory strategies, and discussion of lifestyle factors that impact memory function. Across a series of studies, we have found the group programme to be effective in improving attainment of individual everyday memory goals, prospective memory, strategy use, and confidence in managing memory problems. In a roll-out of the memory skills group to two major stroke services, it was also found to be significantly more cost effective than usual care. This how-to session will outline the content of the Monash Memory Skills Group; explore process issues such as supporting group cohesion, guiding focused group discussion and tailoring content to group members with different needs; outline the key competencies that have been identified as important for running effective groups; and discuss the process of implementing memory skills groups into existing services, including building successful business cases in different contexts. Opportunities for skill practice and development will be provided during this practical, interactive session.

Level aimed for: Intermediate

Learning objectives: Following participation in this how-to session, attendees should be able to:

1. Explain the rationale and evidence for memory skills groups to improve everyday memory in people with conditions affecting the brain.

2. Outline the main content of a manualised memory skills group programme. 
3. Demonstrate understanding of process issues to consider when running a skills-based group with cognitively impaired participants.

4. Practise skills that have been identified as important for running effective memory groups.

5. Describe the issues to be considered in implementing memory skills groups in different clinical settings.

\section{Session 18 - How to Session 6}

\section{Using an Evidence-Based Practice Framework to Guide Support for Decision Making of Adults with Acquired Cognitive and Communication Disability}

Jacinta Douglas, ${ }^{1,2}$ and Lucy Knox ${ }^{1,2}$

${ }^{1}$ La Trobe Univesity, Melbourne, Victoria, Australia

${ }^{2}$ Summer Foundation, Box Hill, Victoria, Australia

ynopsis of session: Around 1 in 45 Australians have acquired brain injury $\mathcal{N}$ (ABI). A considerable number have ongoing cognitive and communication disability requiring significant levels of support for decision-making. To date, the practice of providing support to these people has primarily been based on opinion without the benefit of a systematically developed empirical evidence base. Researchers at the La Trobe University, Living with Disability Research Centre have developed a Support for Decision Making Practice Framework. This framework was derived from the results of a programme of research designed to understand the experience of providing and receiving support for decision making and to establish a sound evidence base upon which to build a practice framework for those who provide support to people with cognitive disabilities.

The framework outlines three elements of the process of support for decision-making including the steps and principles involved and associated strategies for practice. The framework guides those who provide support, whether formally or informally, as family members, health professionals, support workers, or guardians. It can be used to inform thinking about the practice of decision-making support for National Disability Insurance Scheme (NDIS) participants with cognitive disabilities.

Level aimed for: Basic and Intermediate

Learning objectives:

1. Participants will explore contemporary evidence about the provision of support for decision-making for people with cognitive disability.

2. Participants will become familiar with a practice framework to guide the process of support for decision-making with people with cognitive disability and apply this framework to practice based scenarios. 


\title{
ABSTRACTS - SATURDAY POSTERS
}

\section{Computer-Assisted Cognitive Remediation Combined with Strategic Instruction Achieves Greater Improvement in Cognition than an Active Control in Schizophrenia}

\author{
Shayden Bryce, ${ }^{1,2}$ Rirchard Lawrence, ${ }^{1}$ Jennie Ponsford, ${ }^{1,3}$ Susan Rossell, ${ }^{2,4,5}$ Sean \\ Carruthers, ${ }^{2,4}$ Eric Tan, ${ }^{2,4,5}$ and Stuart Lee ${ }^{2,6}$ \\ ${ }^{1}$ School of Psychological Sciences, Monash University, Clayton, VIC, Australia \\ ${ }^{2}$ Monash Alfred Psychiatry Research Centre, The Alfred and Monash University Central Clinical \\ School, Melbourne, VIC, Australia \\ ${ }^{3}$ Monash-Epworth Rehabilitation Research Centre, Richmond, VIC, Australia \\ ${ }^{4}$ Centre for Mental Health, Swinburne University of Technology, Hawthorn, VIC, Australia \\ ${ }^{5}$ Psychiatry, St Vincent's, Fitzroy, VIC, Australia \\ ${ }^{6}$ Department of Psychiatry, Alfred Health, Melbourne, VIC, Australia
}

$\boldsymbol{B}$ ackground and aims: Cognitive impairment is a core feature of B schizophrenia and predicts psychosocial disability. Cognitive remediation (CR) has shown promise for addressing cognitive dysfunction in this illness. A limitation of past research, however, is that CR often occurs alongside other psychosocial interventions, preventing assessment of unique efficacy. Active control conditions and follow-up assessments are also inconsistently utilised. This study explored whether computer-assisted 'drill-and-strategy' cognitive remediation would produce greater improvements in cognition, living skills and self-efficacy than a computer gaming (CG) control.

Method: Fifty-six outpatients with schizophrenia were randomised into CR or CG, and offered 20 one-hour computer sessions over 10 weeks. Cognition, psychiatric symptoms, independent living skills, self-efficacy and quality of life were assessed at baseline, end-group and 3 months follow-up.

Results: Primary analysis was conducted for participants who completed the end-group assessment $(\mathrm{CR}=22$; Control $=21)$. Linear mixed-effect analysis found a significant interaction effect for cognition $(p=.028)$. Bonferroniadjusted pairwise comparisons revealed that cognition was better at end-group and 3 month follow-up than baseline for CR participants (both $p<.01$ ), with no differences between timepoints for control participants. A significant time effect was evident for self-efficacy $(p=.028)$, with both conditions showing higher self-efficacy at end-group than baseline. No other significant effects were observed.

Conclusions: CR combined with internal cognitive strategies may achieve unique improvements in cognition in people with schizophrenia, whereas broader participation in facilitated social groups may achieve improvements in everyday self-efficacy. An explicit focus on the translation of learning experiences may, however, be needed to achieve significant functional improvements.

\section{Outcomes of Patients Participating in a Daily Orientation and Communication Group, in Sub-Acute Acquired Brain Injury Rehabilitation}

Carlo Divita, and Brooke Rendell

State Rehabilitation Service- Acquired Brain Injury Unit, Fiona Stanley Hospital, Murdoch, Western Australia, Australia

$R$ ackground and objectives: In subacute brain injury rehabilitation, groups are used to provide additional opportunities for intervention. A daily group focusing on orientation and communication is run on a 27 bed inpatient ward. The objective of this study is to evaluate the gains that patients 
make in their orientation and communication skills through participation in this group.

Methods: The group is run each morning and follows a structured format including use of the newspaper for orientation, maps for orientation and discussion of newspaper articles. Patients who attend the group are evaluated at commencement and discharge to observe changes in orientation, memory and communication skills. Assessments used include the Story Recall subtest of the Rivermead Behavioural Memory Test, the Modified Communication Checklist, letter verbal fluency test and oLog.

Results: Descriptive statistics are used to characterise the participants in terms of age, length of stay and group participation. Data related to assessment outcomes was entered into SPSS for analysis. Paired t-tests were conducted on initial and discharge scores for Story Recall, letter verbal fluency and oLog scores. Wilcoxon signed rank sum tests were performed on ratings on the Modified Communication Checklist. These changes are compared to the rate of change similar populations experience during rehabilitation, where available in the literature, using one-way ANOVA.

Conclusions: The outcomes provide an indication of the changes which are made by patients over the course of an inpatient rehabilitation stay, of which attendance to a daily orientation and communication group is part.

\title{
Counselling Training for Speech-Language Pathologists to Support People with Post Stroke Aphasia: Methodology
}

\author{
Jasvinder Sekhon, ${ }^{1}$ Jennifer Oates, ${ }^{1}$ Ian Kneebone, ${ }^{2}$ and Miranda Rose ${ }^{1}$ \\ ${ }^{1}$ School of Allied Health, La Trobe University, Melbourne, Australia \\ ${ }^{2}$ Discipline of Clinical Psychology, Graduate School of Health, University of Technology Sydney, \\ Sydney, Australia
}

$\boldsymbol{R}$ ackground and aims: Psychological wellbeing is an important goal in stroke rehabilitation for people with aphasia. There is scarce information on pre-qualification counselling training for speech-language pathologists to support the emotional and psychological wellbeing of this population. Some work places do provide an opportunity to develop counselling skills in collaboration with mental health professionals, and stroke multidisciplinary team.

We aim to report on the development of a counselling training module to improve the competency and confidence of speech-language pathologists to support emotional and psychological wellbeing in people impacted by post stroke aphasia.

Method: A survey of current counselling training for speech-language pathology students at Australian Universities will be conducted. A counselling training module to enable speech-language pathologists working within stroke to support the psychological wellbeing of their clients with post stroke aphasia will be developed, piloted and evaluated.

Results: We anticipate being able to present (a) preliminary results of prequalification counselling training for speech-language pathologists in Australia and (b) key elements of a counselling training module to enable speechlanguage pathologists working within stroke to confidently and competently manage the psychological wellbeing of their clients with post stroke aphasia Conclusions: Appropriate training may improve speech-language pathologist confidence and competence to support emotional and psychological wellbeing in people with post stroke aphasia and their families. 


\title{
Cognitive Profile of Cerebrovascular Disease: From Small Vessel Disease to Chronic Stroke
}

\author{
Maria Shendyapina, ${ }^{1}$ and Brendan S. Weekes ${ }^{1,2}$ \\ ${ }^{1}$ Laboratory for Communication Science, Faculty of Education, University of Hong Kong \\ ${ }^{2}$ School of Psychological Sciences, Faculty of Medicine, Dentistry and Health Sciences, University \\ of Melbourne
}

\begin{abstract}
$\mathcal{B}$ ackground and aims: Cerebrovascular disease (CVD) refers to a vari3 ety of brain dysfunctions that originate from blood vessel impairments. Although CVD is increasing globally, there is no complete model of how it influences cognitive functioning. The aim of the present study is to determine if there is a specific cognitive profile associated with the different stages and neuroanatomical loci of CVD, such as small vessel disease (SVD) and chronic stroke.

Method: 42 patients with SVD and 205 stroke patients aged between 18 and 91 years were assessed with the validated Russian version of the Oxford Cognitive Screen (Rus-OCS) and Montreal Cognitive Assessment (MoCA).

Results: SVD patients had mild levels of cognitive impairments mostly in the memory domain. Over $29 \%$ of participants had deficits on verbal recall, and a further $24 \%$ on the Orientation task. Visual attention (24\%) and praxis (19\%) were also affected. Chronic stroke patients performed significantly worse on language and visiospatial domains with deficits in visual attention $(46 \%)$, verbal memory (35\%), oral reading (34\%), orientation and praxis (30\%), naming, number writing and visual neglect $(21 \%)$.

Conclusion: Cognitive profiles of acute and chronic CVD were compared. The observed differences could be explained by cognitive decline in lesion specific sites following loss of intact brain tissue. Besides this, the accumulation of deficits may lead to the secondary-level cognitive disorders. Timely assessment can inform post CVD care and mitigate further cognitive deterioration with CVD patients.
\end{abstract}

\section{Avoiding Unnecessary Discharge to Residential Aged Care: The Case for a Holistic Community-Based Bridging Solution for Young Adults with Complex Disability}

\author{
Mark Symmons, ${ }^{1,2}$; Jacinta Douglas, ${ }^{1,2}$ and Di Winkler ${ }^{1}$ \\ ${ }^{7}$ Summer Foundation, Box Hill, Australia \\ ${ }^{2}$ Living with Disability Research Centre, La Trobe University, Bundoora, Australia
}

\begin{abstract}
$\boldsymbol{R}$ ackground and aims: In the absence of an existing arrangement to return to, there is a tendency for young adults with a disability to be discharged to residential aged care from hospital. This is rarely an ideal solution for all concerned. Part of a larger project, we present here an alternative discharge planning (DP) framework based on an extensive literature search.

Method: A comprehensive literature search was undertaken, with systematic searching of databases (Pubmed, Cochrane Library, ScienceDirect, PsychInfo), grey literature, and hand searching.

Results: A large body of literature extending across many decades, including systematic reviews, meta analyses and meta systematic reviews, has examined DP for a range of patient categories. Surprisingly, there is a dearth of evaluative literature for DP solutions for young adults with disability and complex care needs. There is ample literature though to enable an understanding of what is needed for an intervention, and why in-ward DP alone tends to produce equivocal results for other complex care needs cohorts and thus is not likely to be effective for the current intervention.
\end{abstract}


Conclusions: A pathway/transition/bridging discharge process is described that: is community-based and thus local and nimble; employs a case management approach; supports the patient and family from soon after hospital admission, through discharge, to a suitable long-term residential arrangement; and is suited to the NDIS environment. The proposed intervention is expected to reduce hospital readmission, be cost-beneficial and elevate client wellbeing.

\section{Post-Traumatic Amnesia Testing: Clinical Practice Patterns Across Australia}

Emma Vo, Anne Gatley, and Sarah Lister

Speech Pathology and Audiology Department, Royal Adelaide Hospital, Adelaide, Australia

$\boldsymbol{R}$ ackground and aims: The accurate measurement of the duration of 0 Post-Traumatic Amnesia (PTA) is well recognised as length of PTA is an indicator of long term deficits and rehabilitation needs. During this challenging and uncertain period, the literature supports early education of family and friends regarding the nature of PTA for support, reassurance and assistance with the management of their loved ones and to facilitate early adjustment. Although a number of local and state guidelines exist for the assessment of PTA, little has been reported about the current practices and consistencies across Australia. The purpose of the current study was to describe current clinical practice patterns across various settings in Australia in relation to PTA testing, and examine these practices with respect to evidence-based practice.

Method: A purpose-built electronic survey was distributed to Occupational Therapy and Speech Pathology national and state-wide interest groups. The survey contained questions relating to clinical practices in the assessment of PTA - including mild Traumatic Brain Injury (TBI) assessment and moderate to very severe TBI assessment as well as management practices for patients in PTA.

Results and conclusions: The poster will describe and discuss national clinical practice patterns in relation to the PTA Testing and management of the patient in PTA and will discuss these findings in relation to current evidence.

\section{Post-Traumatic Amnesia Testing in the Acute Setting: Outcomes Following Implementation of 7-Day Speech Pathology Service}

Emma Vo, Anne Gatley, and Sarah ListerSpeech Pathology and Audiology Department, Royal Adelaide Hospital, Adelaide, Australia

$\boldsymbol{R}$ ackground and aims: Until recently, at our hospital, it was not uncomBon for patients following Closed Head Injury with a suspected or reported loss of consciousness to (1) not have access to Post-Traumatic Amnesia (PTA) testing or (2) not have access to daily PTA testing across 7-days a week and/or (3) have delayed PTA testing. All of these scenarios were reviewed and linked to (1) delayed discharge, (2) delayed transfer to rehabilitation and (3) lack of family, patient and staff education. The purpose of the current study was to describe outcomes following implementation of a 7-day speech pathology service for PTA testing.

Method: A database was kept and analysed using descriptive statistics.

Results: There was a significant improvement in the identification of patients requiring PTA testing with $100 \%$ increase in referrals over a 3-month period. Input resulted in improvement in ensuring suitable follow-up for patients post the acute stage of care, collaboration with rehabilitation services, and family and nursing staff education regarding management of patients in PTA. 
Conclusions: This poster will discuss findings and implications for health care organisations.

\title{
Case Study from A New Multi-Disciplinary Program ("Rewire") for Acquired Brain Injury (ABI)
}

Edward Weaver, Naomi Jarvis, ${ }^{1}$ and Richard Clark ${ }^{2}$

${ }^{1}$ Brain Injury SA, Adelaide, SA

${ }^{2}$ Brain Clinics Australia and Flinders University, Adelaide, SA

$\boldsymbol{R}$ ackground and objectives: Rewire is a multidisciplinary programme for ABI, aiming to help people achieve their goals and adjust to life post-injury.

Jim (26 years) sustained a severe traumatic brain injury (TBI) in a motorcycle accident in January 2017 (GCS 6/15, PTA 58 days, MRI indications of DAI). He completed Rewire between April and September 2017. Initial QEEG and neuropsychological assessment revealed low voltage EEG indicating brain underactivation, cognitive and communication impairment, as well as depression and anxiety in the clinical range. Jim's goals were to regain his driver's license, normalise speech and return to work, sport and exercise. Jim's programme comprised speech pathology, occupational therapy, psychology, neurotherapy, physiotherapy, exercise physiology and dietetics.

Method: Cognitive, affective and brain function were examined upon commencing Rewire and at completion using neuropsychological assessment and QEEG, with ongoing qualitative assessment of progress.

Results: Neuropsychological assessment revealed that depression and anxiety normalised, as did issues with psychomotor speed, learning, memory and executive function. QEEG data suggested that brain underactivation improved significantly. Some residual difficulties with cortico-cortical connectivity remained. Jim met all his goals. He valued the programme's flexibility, team effort and its focus on his goals.

Conclusion: The adaptability of the programme and its collaborative approach appeared to have contributed to outcomes achieved. There were indications that neurotherapy and psychotherapy may have provided a synergistic influence on the normalisation of brain and psychological function. The clinical case study design, and in particular the lack of a control group, limits the extent to which results can be generalised. We recommend rigorous studies with appropriate controls to further assess the efficacy of Rewire.

\section{Exploration of Structured Communication During Client-Centred Goal Setting with People with Acquired Brain Injury}

\author{
Nicole Weir, ${ }^{1}$ Sarah Prescott, ${ }^{2}$ Jennifer Fleming, ${ }^{2}$ and Emmah Doig ${ }^{2}$ \\ ${ }^{\prime}$ Occupational Therapy Department, Princess Alexandra Hospital, Brisbane, Australia \\ ${ }^{2}$ School of Health and Rehabilitation Sciences, The University of Queensland, Brisbane, Australia
}

\footnotetext{
$B$ ackground and objectives: Client-centred goal setting with individuals with acquired brain injury (ABI) can be challenging, particularly when clients experience cognitive and communication impairment. To address this need, we explored the application of the goal setting practice framework in routine practice, a framework developed from therapist interview to explain how achievable client- centred goals may be developed for individuals with ABI. Structured communication was identified as a strategy used to engage clients in goal setting across all interviews. This study aimed to understand implementation of structured communication as a strategy in practice.
} 
Method: This qualitative observational study recruited participants with ABI, from an outpatient hospital-based service and community-based private practices. Communication between multidisciplinary clinicians and clients during routine goal setting was audio-recorded, transcribed verbatim and analysed using Framework Analysis. In total, 66 goal setting sessions with 37 clients and 18 clinicians were analysed. Independent coding and fortnightly consensus meetings enhanced rigour.

Results: In most cases, therapists structured their communication from the beginning of the goal setting session and used this approach during all discussions with the client. Structured communication involved using concrete concepts to assist client understanding, prompting clients to self-reflect about the functional impact of their impairments. This strategy was also used to educate clients about the way therapy activities addressed rehabilitation needs. Formal goal setting tools also enabled therapists to structure their communication.

Conclusion: Structured communication may be used to enhance client-centred goal setting in ABI rehabilitation.

\title{
Factors Impacting on Selection and Use of Cognitive Assessments by Occupational Therapists Working in an Inpatient Setting
}

\author{
Jacqueline A.Wheatcroft, ${ }^{1}$ Rebecca J. Nicks, ${ }^{1}$ Laura Jolliffe, ${ }^{1,2}$ and Natasha A. \\ Lannin, ${ }^{1,2}$ \\ ${ }^{1}$ Occupational Therapy Department, Alfred Health, Melbourne, Australia \\ ${ }^{2}$ School of Allied Health (Occupational Therapy), College of Science, Health \& Engineering, La Trobe \\ University, Melbourne, Australia
}

$\boldsymbol{R}$ ackground and objectives: Screening and assessment of cognitive ability for persons admitted with neurological impairments assists with rehabilitation and discharge planning. Translating international guidelines and local policies into clinical practice is essential to this process. However, little is understood about the decision-making for selecting and using cognitive assessments from the therapists' perspective.

The aim of this project was to identify potential barriers and enablers to occupational therapists applying guideline recommendations for cognitive assessment in the acute and subacute hospital setting.

Method: Cross-sectional study within one area health network. An on-line survey based on the Theoretical Domains Framework (TDF) and COM-B ('capability', 'opportunity', 'motivation' and 'behaviour') model was sent out to $N=81$ occupational therapists across the organisation.

Results: Forty occupational therapists (49\%) responded to the survey. Participants identified cognitive assessment as a core component of their role, however, analysis using the COM-B showed barriers in the domains of Capability (Knowledge (TDF): capability to plan rehabilitation from the results) and Motivation (Goal (TDF): other assessments/ interventions seeming more urgent; Reinforcement (TDF): reinforcement and recognition for completing assessments; Optimism (TDF): about completing the assessment; Beliefs about Capability(TDF): administering assessments under time pressures or the patient is not motivated).

Conclusions: Our findings advance understanding of what facilitates and inhibits occupational therapists' adherence to guidelines in relation to cognitive assessment. Using the TDF and COM-B model provide a useful conceptual basis to identify challenges which affect the implementation of guideline recommendations, and will support the development of quality assurance interventions to improve adherence in clinical practice. 


\section{Modifiers of Sport Concussion Assessment Tool 3 (SCAT-3) Performance in Non-Contact Sports Athletes}

Ashley Paxton, ${ }^{1}$ and Catherine Willmott ${ }^{1,2}$

${ }^{1}$ School of Psychological Sciences, Monash Institute of Cognitive and Clinical Neurosciences, Monash University, Melbourne, Australia

${ }^{2}$ Monash-Epworth Rehabilitation Research Centre, Melbourne, Australia

$\boldsymbol{B}$ ackground and aims: The Sport Concussion Assessment Tool 3 (SCAT3 ) is considered the most sensitive and reliable screen for sports concussion, however, little is known about potential modifiers of SCAT-3 performance. Research suggests addition of the King-Devick (K-D), an ocular functioning assessment, improves efficacy of the SCAT-3 in identifying concussion. This study examined whether exercise or practice influenced SCAT-3 or K-D performance, and assessed whether an association exists between mood, pain and SCAT-3 symptom scoring in a non-concussed sample.

Method: Participants $n=16$ male and $n=14$ female (Mage $=22.73$, SDage $=3.59)$ moderately active non-contact sports athletes. A crossover repeated-measures design with two time periods (T1 and T2), four weeks apart, and participants allocated to two groups was utilised. SCAT-3 and K-D were administered at both time periods, whilst the exercise protocol was administered only once (T1 for Group $1 \& \mathrm{~T} 2$ for Group 2), 15 minutes prior to the SCAT-3 and K-D. Participants also completed the Depression, Anxiety and Stress Scale short-form and the Brief Pain Inventory.

Results: Exercise did not influence SCAT-3 or K-D results. Practice effects were shown on the K-D, but not on SCAT-3 cognitive and physical assessments. Higher levels of mood disturbance were associated with greater symptom reporting. Pain showed no correlation with symptom report.

Conclusions: Findings assist in validating the SCAT-3 for use in evaluating changes associated with sports concussion, in conjunction with the KingDevick, and suggest caution is needed when interpreting the SCAT- 3 in athletes with mood disturbance. 